\title{
Identification and Estimation of Games with Incomplete Information Using Excluded Regressors ${ }^{1}$
}

\author{
Arthur Lewbel, Boston College \\ Xun Tang, University of Pennsylvania
}

Original: November 25, 2010. Revised: March 5, 2013

\begin{abstract}
The existing literature on binary games with incomplete information assumes that either payoff functions or the distribution of private information are finitely parameterized to obtain point identification. In contrast, we show that, given excluded regressors, payoff functions and the distribution of private information can both be nonparametrically point identified. An excluded regressor for player $i$ is a sufficiently varying state variable that does not affect other players' utility and is additively separable from other components in $i$ 's payoff. We show how excluded regressors satisfying these conditions arise in contexts such as entry games between firms, as variation in observed components of fixed costs. Our identification proofs are constructive, so consistent nonparametric estimators can be readily based on them. For a semiparametric model with linear payoffs, we propose root- $\mathrm{N}$ consistent and asymptotically normal estimators for parameters in players' payoffs. Finally, we extend our approach to accommodate the existence of multiple Bayesian Nash equilibria in the data-generating process without assuming equilibrium selection rules.
\end{abstract}

Keywords: Games with Incomplete Information, Excluded Regressors, Nonparametric Identification, Semiparametric Estimation, Multiple Equilibria.

JEL Codes: C14, C51, D43

\footnotetext{
${ }^{1}$ We thank Arie Beresteanu, Aureo de Paula, Stefan Hoderlein, Zhongjian Lin, Pedro Mira, Jean-Marc Robin, Frank Schorfheide and Kenneth Wolpin for helpful discussions. We also thank three anonymous referees and seminar paricipants at Asian Econometric Society Summer Meeting 2011, Brown U, Duke U, London School of Economics, Rice U, U British Columbia, U Conn, U Penn and U Pitts for feedbacks and comments. All errors are our own.
} 


\section{Introduction}

We estimate a class of static game models where players simultaneously choose between binary actions based on imperfect information about each other's payoffs. Such a class of models lends itself to wide applications in industrial organization. Examples include firms' entry decisions in Seim (2006), decisions on the timing of commercials by radio stations in Sweeting (2009), firms' choice of capital investment strategies in Aradillas-Lopez (2010) and interactions between equity analysts in Bajari, Hong, Krainer and Nekipelov (2010).

\section{- Preview of Our Method}

We introduce a new approach to identify and estimate such models when players' payoffs depend on a vector of "excluded regressors". An excluded regressor associated with a given player $i$ is a state variable that does not enter payoffs of the other players, and is additively separable from other components in $i$ 's payoff. We show that if excluded regressors are orthogonal to players' private information given observed states, then interaction effects between players and marginal effects of excluded regressors on players' payoffs can be identified. If in addition the excluded regressors vary sufficiently relative to the support of private information, then the entire payoff functions of each player and the distribution of players' private information are nonparametrically identified. No distributional assumption on the private information is necessary for these results. Our identification proofs are constructive, so consistent nonparametric estimators can be readily based on them.

We provide an example of an economic application in which profit maximization by players gives rise to excluded regressors with the required properties. Consider the commonly studied game in which firms each simultaneously decide whether to enter a market or not, knowing that after entry they will compete through choices of quantities. The fixed cost for a firm $i$ (to be paid upon entry) does not affect its perception of the interaction effects with other firms, or the difference between its monopoly profits (when $i$ is the sole entrant) and oligopoly profits (when $i$ is one of the multiple entrants). This is precisely the independence restriction required of excluded regressors.

The intuition for this result is that fixed costs drop out of the first-order conditions in the profit maximization problems both for a monopolist and for oligopolists engaging in Cournot competition. As result, while fixed costs affect the decision to enter, they do not otherwise affect the quantities supplied by any of the players. In practice, only some components of a firm's fixed cost are observed by econometricians, while the remaining, unobserved part of the fixed cost is the private information only known to this firm. Our method can thus be applied, with the observed component of fixed costs playing the role of excluded regressors.

Other excluded regressor conditions that contribute to identification (such as conditional in- 
dependence from private information and sufficient variation relative to the conditional support of private information given observed states) also have immediate economic interpretations in the context of entry games. We discuss those interpretations in greater details in Section 4.

We extend our excluded regressor approach to obtain point identification while allowing for multiple Bayesian Nash equilibria (BNE) in the data generating process (DGP), without assuming knowledge of an equilibrium selection mechanism. Identification is obtained as before, but only using variation of excluded regressors within a set of states with a single BNE in the data-generating process. We build on De Paula and Tang (2011), showing that when players' private information is independent conditional on observed states, then the players' actions are correlated given these states if and only if those states give rise to multiple BNE. Thus it is possible to infer from the data whether a given set of states give rise to single BNE, and base identification only on those states, without imposing parametric assumptions on equilibrium selection mechanisms or on the distribution of private information.

\section{- Relation to Existing Literature}

This paper contributes to the existing literature on structural estimation of Bayesian games in several ways. First, we identify the complete structure of the model under a set of mild nonparametric restrictions on primitives. This is in contrast to the existing literature, which requires that either the payoff functions or the distribution of private information be finitely parameterized to obtain point identification.

Bajari, Hong, Nekipelov and Krainer (2010) identify the players' payoffs in a general model of multinomial choices, without assuming any particular functional form for payoffs, but they require the researcher to have full knowledge of the distribution of private information. In contrast we allow the distribution of private information to be unknown and nonparametric. We instead obtain identification by imposing economically motivated exclusion restrictions.

Sweeting (2009) proposes a maximum-likelihood estimator where players' payoffs are linear indices and the distribution of players' private information is known to belong to a parametric family. Aradillas-Lopez (2010) estimates a model with linear payoffs when players' private information are independent of states observed by researchers. He also proposes a root- $\mathrm{N}$ consistent estimator by extending the semi-parametric estimator for single-agent decision models in Klein and Spady (1993) to the game-theoretic setup. Tang (2010) shows identification of a similar model to Aradillas-Lopez (2010), with the independence between private information and observed states replaced by a weaker median independence assumption, and proposes consistent estimators for linear coefficients without establishing rates of convergence. Wan and Xu (2010) consider a class of Bayesian games with index utilities under median restrictions. They allow players' private information to be positively correlated in a particular form, and focus on a class of monotone, threshold crossing pure-strategy equilibria. Unlike these papers, we show non-parametric identification of players' payoffs, with no 
functional forms imposed. We also allow private information to be correlated with non-excluded regressors in unrestricted ways but require them to be independent across players conditional on observable states.

The second contribution of this paper is to propose a two-step, consistent and asymptotically normal estimator for semi-parametric models with linear payoffs and private information correlated with non-excluded regressors. If in addition the support of private information and excluded regressors are bounded, our estimators for the linear coefficients in baseline payoffs attain the parametric rate. If these supports are unbounded, then the rate of convergence of estimators for these coefficients depends on properties of the tails of the distribution of excluded regressors. The estimators for interaction effects converge at the parametric rate regardless of the boundedness of these supports. $^{2}$

Our third contribution is to accommodate multiple Bayesian Nash equilibria without relying on knowledge of any equilibrium selection mechanism. The possibility of multiple BNE in data poses a challenge to structural estimation because in this case observed player choice probabilities are an unknown mixture of those implied by each of the multiple equilibria. Hence, the exact structural link between the observable distribution of actions and model primitives is not known. The existing literature proposes several approaches to address this issue. These include parameterizing the equilibrium selection mechanism, assuming only a single BNE is followed by the players (Bajari, Hong, Krainer and Nekipelov (2010)), introducing sufficient restrictions on primitives to ensure that the system characterizing the equilibria have a unique solution (Aradillas-Lopez (2010)), or only obtaining partial identification results (Beresteanu, Molchanov, Molinari (2011)). ${ }^{3}$

For a model of Bayesian games with linear payoffs and correlated bivariate normal private information, $\mathrm{Xu}$ (2009) addressed the multiplicity issue by focusing on states for which the model only admits a unique, monotone equilibrium. He showed, with these parametric assumptions, a subset of these states can be inferred using the observed distribution of choices and then used for estimating parameters in payoffs.

Our approach for dealing with multiple BNE builds on a related idea. Instead of focusing on states where the model only permits a unique equilibrium, we focus on a set of states where the observed choice probabilities are rationalized by a unique BNE. This set can include both states

\footnotetext{
${ }^{2}$ Khan and Nekipelov (2011) studied the Fisher information for the interaction parameter in a similar model of Bayesian games, where private information are correlated across players, but the baseline payoff fuctions are assumed known. Unlike our paper, the goal of their paper is not to jointly identify and estimate the full structure of the model. Rather, they focus on showing regular identification (i.e. positive Fisher information) of the interaction parameter while assuming knowledge of the functional form of the rest of the payoffs as well as the equilibrium selection mechanism.

${ }^{3}$ Aguirregabiria and Mira (2005) propose an estimator for payoff parameters in games with multiple equilibria where identification is assumed. Their estimator combines a pseudo-maximum likelihood procedure with a genetic algorithm that searches globally over the space of possible combinations of multiple equilibria in the data.
} 
where the model admits a unique BNE only, and states where multiple equilibria are possible but where the agents in the data always choose the same BNE. We identify such states without parametric restrictions on primitives and without equilibrium selection assumptions. Applying our excluded regressor assumptions to a set of states where the observed choice probabilities is rationalized by a unique BNE then allows us to identify the model nonparametrically.

Our use of excluded regressors is related to the use of "special regressors" in single-agent, qualitative response models (See Dong and Lewbel (2011) and Lewbel (2012) for surveys of special regressor estimators). Lewbel $(1998,2000)$ studies nonparametric identification and estimation of transformation, binary, ordered, and multinomial choice models using a special regressor that is additively separable from other components in decision-makers' payoffs, is independent from unobserved heterogeneity conditional on other covariates, and has a large support. Magnac and Maurin (2008) study partial identification of the model when the special regressor is discrete or measured within intervals. Magnac and Maurin (2007) remove the large support requirement on the special regressor in such a model, and replace it with an alternative symmetry restriction on the distribution of the latent utility. Lewbel, Linton, and McFadden (2011) estimate features of willingness-to-pay models with a special regressor chosen by experimental design. Berry and Haile $(2009,2010)$ extend the use of special regressors to nonparametrically identify the market demand for differentiated goods.

Special regressors and exclusion restrictions have also been used for estimating a wider class of models including social interaction models and games with complete information. Brock and Durlauf (2007) and Blume, Brock, Durlauf and Ioannides (2010) use exclusion restrictions of instruments to identify a linear model of social interactions. Bajari, Hong and Ryan (2010) use exclusion restrictions and identification at infinity, while Tamer (2003) and Ciliberto and Tamer (2009) use some special regressors to identify games with complete information. Our identification differs qualitatively from that of complete information games because with incomplete information, each player-specific excluded regressor can affect the strategies of all other players' through its impact on the equilibrium choice probabilities.

\section{- Roadmap}

The rest of the paper proceeds as follows. Section 2 introduces the model of discrete Bayesian gams. Section 3 establishes nonparametric identification of the model. Section 4 motivates our key identifying assumptions using an example of entry games, where observed components of each firm's fixed cost lends itself to the role of excluded regressors. Sections 5 and 6 propose estimators for a semiparametric model where players' payoffs are linear. Section 7 provides some evidence of the finite sample performance of this estimator. Section 8 extends our identification method to models with multiple equilibria, including testing whether observed choices are rationalizable by a single BNE over a subset of states in the data-generating process. Section 9 concludes. 


\section{The Model}

Consider a model of simultaneous games with binary actions and incomplete information that involves $N$ players. We use $N$ to denote both the set and the number of players. Each player $i$ chooses an action $D_{i} \in\{1,0\}$. A vector of states $X \in \mathbb{R}^{J}$ with $J>N$ is commonly observed by all players and the econometrician. Let $\epsilon^{*} \equiv\left(\epsilon_{i}^{*}\right)_{i=1}^{N} \in \mathbb{R}^{N}$ denote players' private information, where $\epsilon_{i}^{*}$ is observed by $i$ but not anyone else. Throughout the paper we use upper cases (e.g. $\left.X, \epsilon_{i}^{*}\right)$ for random vectors and lower cases (e.g. $x, \varepsilon_{i}^{*}$ ) for realized values. For any pair of random vectors $R$ and $R^{\prime}$, let $\Omega_{R^{\prime} \mid R}, f_{R^{\prime} \mid R}$ and $F_{R^{\prime} \mid R}$ denote respectively the support, the density and the distribution of $R^{\prime}$ conditional on $R$.

The payoff for $i$ from choosing action 1 is $\left[u_{i}^{*}\left(X, \epsilon_{i}^{*}\right)+h_{i}\left(D_{-i}\right) \delta_{i}^{*}(X)\right] D_{i}$ where $D_{-i} \equiv\left\{D_{j}\right\}_{j \neq i}$ and $h_{i}($.$) is bounded and common knowledge among all players and known to the econometrician. { }^{4}$ The payoff from choosing action 0 is normalized to 0 for all players. Here $u_{i}^{*}\left(X, \epsilon_{i}^{*}\right)$ denotes the baseline return for $i$ from choosing 1 , while $h_{i}\left(D_{-i}\right) \delta_{i}^{*}(X)$ captures interaction effects, i.e., how other players' actions affect $i$ 's payoff. One example of $h_{i}$ is $h_{i}\left(D_{-i}\right) \equiv \sum_{j \neq i} D_{j}$, making the interaction effect proportional to the number of competitors choosing 1, as in Sweeting (2009) and Aradillas-Lopez (2010). Other examples of $h_{i}\left(D_{-i}\right)$ include $\min _{j \neq i} D_{-i}$ or $\max _{j \neq i} D_{-i}$. We require the interaction effect $h_{i}\left(D_{-i}\right) \delta_{i}^{*}(X)$ to be multiplicatively separable in observable states $X$ and rivals' actions $D_{-i}$, but $\delta_{i}^{*}$ and the baseline $u_{i}^{*}$ are allowed to depend on $X$ in general ways. The model primitives $\left\{u_{i}^{*}, \delta_{i}^{*}\right\}_{i=1}^{N}$ and $F_{\epsilon^{*} \mid X}$ are common knowledge among players but are not known to the econometrician. We maintain the following restrictions on $F_{\epsilon^{*} \mid X}$ throughout the paper.

A1 (Conditional Independence) Given any $x \in \Omega_{X}, \epsilon_{i}^{*}$ is independent from $\left\{\epsilon_{j}^{*}\right\}_{j \neq i}$ for all $i$, and $F_{\epsilon_{i}^{*} \mid x}$ is absolutely continuous with respect to the Lebesgue measure and has positive Radon-Nikodym densities almost everywhere over $\Omega_{\epsilon_{i}^{*} \mid x}$.

This assumption, which allows $X$ to be correlated with players' private information, is maintained in almost all previous works on the estimation of Bayesian games (e.g. Seim (2006), AradillasLopez (2010), Berry and Tamer (2007), Bajari, Hong, Krainer, and Nekipelov (2010), Bajari, Hahn, Hong and Ridder (2010), Brock and Durlauf (2007), Sweeting (2009) and Tang (2010)). ${ }^{5}$ Throughout this paper, we assume players adopt pure strategies only. A pure strategy for $i$ in a game with $X$ is a mapping $s_{i}(X,):. \Omega_{\epsilon_{i}^{*} \mid X} \rightarrow\{0,1\}$. Let $s_{-i}\left(X, \epsilon_{-i}^{*}\right)$ represent a profile of $N-1$ strategies of $i$ 's competitors $\left\{s_{j}\left(X, \epsilon_{j}^{*}\right)\right\}_{j \neq i}$. Under A1, any Bayes Nash equilibrium (BNE) under states $X$ must

${ }^{4}$ With $N=2$, assuming $h_{i}\left(D_{j}\right)=D_{j}$ does not induce any loss of generality. With $N \geq 3$, assuming $h_{i}$ is known to the researcher is restrictive. Bajari, Hong, Krainer, and Nekipelov (2010) avoid this assumption, and allow the payoff matrix to be completely nonparametric, but they require the assumption that the distribution of unobserved states is completely known to the econometrician.

${ }^{5} \mathrm{~A}$ recent exception is Wan and $\mathrm{Xu}$ (2010), who nevertheless restrict equilibrium strategies to be monotone with a threshold-crossing property, and impose restrictions on the magnitude of the strategic interaction parameters. 
be characterized by a profile of strategies $\left\{s_{i}(X, .)\right\}_{i \leq N}$ such that for all $i$ and $\epsilon_{i}^{*}$,

$$
s_{i}\left(X, \epsilon_{i}^{*}\right)=1\left\{u_{i}^{*}\left(X, \epsilon_{i}^{*}\right)+\delta_{i}^{*}(X) E\left[h_{i}\left(s_{-i}\left(X, \epsilon_{-i}^{*}\right)\right) \mid X, \epsilon_{i}^{*}\right] \geq 0\right\} .
$$

The expectation in (1) is conditional on $i$ 's information $\left(X, \epsilon_{i}^{*}\right)$ and is taken with respect to private information of his rivals $\epsilon_{-i}^{*}$. By A1, $E\left[h_{i}\left(D_{-i}\right) \mid X, \epsilon_{i}^{*}\right]$ must be a function of $X$ alone. Thus in any $\mathrm{BNE}$, the joint distribution of $D_{-i}$ conditional on $i$ 's information $\left(x, \varepsilon_{i}^{*}\right)$ and the profile of strategies takes the form

$$
\operatorname{Pr}\left\{D_{-i}=d_{-i} \mid x, \varepsilon_{i}^{*}\right\}=\operatorname{Pr}\left\{s_{-i}\left(X, \epsilon_{-i}^{*}\right)=d_{-i} \mid x\right\}=\Pi_{j \neq i}\left(p_{j}(x)\right)^{d_{j}}\left(1-p_{j}(x)\right)^{1-d_{j}},
$$

for all $d_{-i} \in\{0,1\}^{N-1}$, where $p_{i}(x) \equiv \operatorname{Pr}\left\{u_{i}^{*}\left(X, \epsilon_{i}\right)+\delta_{i}^{*}(X) E\left[h_{i}\left(s_{-i}\left(X, \epsilon_{-i}\right)\right) \mid X\right] \geq 0 \mid X=x\right\}$ is $i$ 's probability of choosing 1 given $x$.

For the special case where $h_{i}\left(D_{-i}\right)=\sum_{j \neq i} D_{j}$ for all $i$, the profile of BNE strategies can be characterized by a vector of choice probabilities $\left\{p_{i}(x)\right\}_{i \leq N}$ such that:

$$
p_{i}(x)=\operatorname{Pr}\left\{u_{i}^{*}\left(X, \epsilon_{i}^{*}\right)+\delta_{i}^{*}(X) \sum_{j \neq i} p_{j}(X) \geq 0 \mid X=x\right\} \text { for all } i \in N .
$$

Note this characterization only involves a vector of marginal rather than joint probabilities. The existence of pure-strategy BNE given any $x$ follows from the Brouwer's Fixed Point Theorem and the continuity of $F_{\epsilon^{*} \mid X}$ in A1.

In general, the model can admit multiple BNE since the system (3) could admit multiple solutions. So in the DGP players' choices observed under some state $x$ could potentially be rationalized by more than one BNE. In Sections 3 and 6, we first identify and estimate the model assuming players' choices are rationalized by a single BNE for each state $x$ in data (either because their specific model only admits unique equilibrium, or because agents employ some equilibrium selection mechanism possibly unknown to the researcher). Later in Section 8 we extend our use of excluded regressors to obtain identification under multiple BNE. This organization of the paper allows us to separate our main identification strategy from the added complications involved in dealing with multiple equilibria, and permits direct comparison with existing results that assume a single BNE, such as Bajari, Hong, Krainer and Nekipelov (2010), Aradillas-Lopez (2010) and Wan and Xu (2010).

\section{Nonparametric Identification}

We consider nonparametric identification assuming a large cross-section of independent games, each involving $N$ players, with the fixed structure $\left\{u_{i}^{*}, \delta_{i}^{*}\right\}_{i \in N}$ and $F_{\epsilon^{*} \mid X}$ underlying all games observed 
in data. ${ }^{6}$ The econometrician observes players' actions and the states in $x$, but does not observe $\left\{\varepsilon_{i}^{*}\right\}_{i \in N}$.

A2 (Unique Equilibrium) In data, players' choices under each state $x$ are rationalized by a single BNE at that state.

This assumption will be relaxed later, essentially by restricting attention to a subset of states for which it holds. For each state $x$ A2 could hold either because the model only admits a unique BNE under that state, or because the equilibrium selection mechanism is degenerate at a single BNE under that state. Without A2, the joint distribution of choices observable from data would be a mixture of several choice probabilities, with each implied by one of the multiple BNE. The key role of A2 is to ensure the vector of conditional expectations of $h_{i}\left(D_{-i}\right)$ given $X$ as directly identified from data satisfies the system in (1)-(2). When $h\left(D_{-i}\right)=\sum_{j \neq i} D_{j}$, A2 ensures the conditional choice probabilities of each player (denoted by $\left\{p_{i}^{*}(x)\right\}_{i \in N}$ ) as directly identified from data can solve (3). As such, (3) reveals how the variation in excluded regressors affects the observed choice probabilities, which provides the foundation of our identification strategies.

A3 (Excluded Regressors) The J-vector of states in $x$ is partitioned into two vectors $x_{e} \equiv\left(x_{i}\right)_{i \in N}$ and $\tilde{x}$ such that (i) for all $i$ and $\left(x, \varepsilon_{i}^{*}\right), \delta_{i}^{*}(x)=\delta_{i}(\tilde{x})$ and $u_{i}^{*}\left(x, \varepsilon_{i}^{*}\right)=\alpha_{i} x_{i}+u_{i}\left(\tilde{x}, \varepsilon_{i}^{*}\right)$ for $\alpha_{i} \in$ $\{-1,1\}$ and some unknown functions $u_{i}$ and $\delta_{i}$, where $u_{i}$ is continuous in $\epsilon_{i}^{*}$ at all $\tilde{x}$; (ii) for all $i$, $\epsilon_{i}^{*}$ is independent from $X_{e}$ given any $\tilde{x}$; and (iii) given any $\tilde{x}$, the distribution of $X_{e}$ is absolutely continuous w.r.t. the Lebesgue measure and has positive Radon-Nikodym densities a.e. over the support.

Hereafter we refer to the $N$-vector $X_{e} \equiv\left(X_{i}\right)_{i \in N}$ as excluded regressors, and the remaining ( $J-$ $N)$-vector of regressors $\tilde{X}$ as non-excluded regressors. The main restriction on excluded regressors is that they do not appear in the interaction effects $\delta_{i}^{*}(x)$, and each only appears additively in one baseline payoff $u_{i}^{*}\left(x, \varepsilon_{i}^{*}\right)$. The scale of each coefficient $\alpha_{i}$ in the baseline payoff $u_{i}^{*}\left(x, \varepsilon_{i}^{*}\right)=$ $\alpha_{i} x_{i}+u_{i}\left(\tilde{x}, \varepsilon_{i}^{*}\right)$ is normalized to $\left|\alpha_{i}\right|=1$. This is the free scale normalization that is available in all binary choice threshold crossing models.

A3 extends the notion of continuity and conditional independence of a scalar special regressor in single-agent models as in Lewbel $(1998,2000)$ to the context with strategic interactions between multiple decision-makers. Identification using excluded regressors in $X_{e}$ in the game-theoretic setup differs qualitatively from the use of special regressors in single-agent cases, because in any equilibrium $X_{e}$ still affect all players' decisions through its impact on the joint distribution of actions.

\footnotetext{
${ }^{6}$ It is not necessary for all games observed in data to have the same group of physical players. Rather, it is only required that the $N$ players in the data have the same underlying set of preferences given by $\left(u_{i}^{*}, \delta_{i}^{*}\right)_{i \in N}$.
} 


\subsection{Identification overview and intuition}

To summarize our identification strategy, consider the special case of $N=2$ players indexed by $i \in\{1,2\}$. When we refer to one player as $i$, the other will be denoted $j$. For this special case assume $h_{i}\left(D_{-i}\right)=D_{j}$. Here the vector $x$ consists of the excluded regressors $x_{1}$ and $x_{2}$ along with the vector of remaining, non-excluded regressors $\tilde{x}$. Let $\mathcal{S}_{i} \equiv-u_{i}\left(\tilde{X}, \epsilon_{i}^{*}\right)$, where $u_{i}\left(\tilde{X}, \epsilon_{i}^{*}\right)$ is the part of player $i$ 's payoffs that does not depend on excluded regressors, and let $F_{\mathcal{S}_{i} \mid \tilde{x}}$ denote the conditional c.d.f. of $\mathcal{S}_{i}$.

Given A1,2,3, the equation (3) simplifies in this two player case to

$$
p_{i}^{*}(x)=F_{\mathcal{S}_{i} \mid \tilde{x}}\left[\alpha_{i} x_{i}+\delta_{i}(\tilde{x}) p_{j}^{*}(x)\right] .
$$

Let $p_{i, j}^{*}(x) \equiv \partial p_{i}^{*}(X) /\left.\partial X_{j}\right|_{X=x}$. Taking partial derivatives of equation (4) with respect to $x_{i}$ and $x_{j}$ gives

$$
\begin{gathered}
p_{i, i}^{*}(x)=\left[\alpha_{i}+\delta_{i}(\tilde{x}) p_{j, i}^{*}(x)\right] \tilde{f}_{i}(x) \\
p_{i, j}^{*}(x)=\delta_{i}(\tilde{x}) p_{j, j}^{*}(x) \tilde{f}_{i}(x)
\end{gathered}
$$

where $\tilde{f}_{i}(x)$ is the density of $\mathcal{S}_{i} \mid \tilde{x}$ evaluated at $\alpha_{i} x_{i}+\delta_{i}(\tilde{x}) p_{j}^{*}(x)$. Since choices are observable, choice probabilities $p_{i}^{*}(x)$ and their derivatives $p_{i, j}^{*}(x)$ are identified.

The first goal is to identify the signs of the excluded regressor marginal effects $\alpha_{i}$ (recalling that their magnitudes are normalized to one) and the interaction terms $\delta_{i}(\tilde{x})$. Solve equation (6) for $\delta_{i}(\tilde{x})$, substitute the result into equation (5), and solve for $\alpha_{i}$ to obtain $\alpha_{i} \tilde{f}_{i}(x)=$ $p_{i, i}^{*}(x)-p_{i, j}^{*}(x) p_{j, i}^{*}(x) / p_{j, j}^{*}(x)$. Since the density $\tilde{f}_{i}(x)$ must be nonnegative and somewhere strictly positive, this identifies the sign $\alpha_{i}$ in terms of identified choice probability derivatives. Next, the interaction term $\delta_{i}(\tilde{x})$ is identified by dividing equation (5) by equation (6) to obtain $p_{i, i}^{*}(x) / p_{i, j}^{*}(x)=$ $\left[\alpha_{i}+\delta_{i}(\tilde{x}) p_{j, i}^{*}(x)\right] / \delta_{i}(\tilde{x}) p_{j, j}^{*}(x)$, and solving this equation for $\delta_{i}(\tilde{x})$.

A technical condition called NDS that we provide later defines a set $\omega$ of values of $x$ for which these constructions can be done avoiding the subtle problem of division by zero. Estimation of $\alpha_{i}$ and $\delta_{i}(\tilde{x})$ can directly follow these constructions, based on nonparametric regression estimates of $p_{i}^{*}(x)$, then averaging the expression for $\alpha_{i}$ over all $x \in \omega$ for $\alpha_{i}$ and averaging the expression for $\delta_{i}(\tilde{x})$ over values the excluded regressors take on in $\omega$ conditional on $\tilde{x}$. This averaging exploits over-identifying information in our model to increase efficiency.

The remaining step is to identify the distribution function $F_{\mathcal{S}_{i} \mid \tilde{x}}$ and its features. For each $i$ and $x$, define a "generated special regressor" as $v_{i} \equiv V_{i}(x) \equiv \alpha_{i} x_{i}+\delta_{i}(\tilde{x}) p_{j}^{*}(x)$. These $V_{i}(x)$ are identified since $\alpha_{i}$ and $\delta_{i}(\tilde{x})$ have been identified. It then follows from the BNE and equation (1) that each player $i$ 's strategy is to choose

$$
D_{i}=1\left\{\alpha_{i} X_{i}+\delta_{i}(\tilde{X}) p_{j}^{*}(X)+u_{i}\left(\tilde{X}, \epsilon_{i}^{*}\right) \geq 0\right\}=1\left\{V_{i} \geq \mathcal{S}_{i}\right\} .
$$


This has the form of a binary choice threshold crossing model, with unknown function $\mathcal{S}_{i}=$ $-u_{i}\left(\tilde{X}, \epsilon_{i}^{*}\right)$ and error $\epsilon_{i}^{*}$ having an unknown distribution with unknown heteroskaedasticity. It follows from the excluded regressors $X_{e}$ being conditionally independent of $\epsilon_{i}$ that $V_{i}$ and $\mathcal{S}_{i}$ are conditionally independent of each other, conditioning on $\tilde{x}$. The main insight is that therefore $V_{i}$ takes the form of a special regressor as in Lewbel $(1998,2000)$, which provides the required identification. In particular, the conditional independence makes $E\left(D_{i} \mid v_{i}, \tilde{x}\right)=F_{\mathcal{S}_{i} \mid \tilde{x}}\left(v_{i}\right)$, so $F_{\mathcal{S}_{i} \mid \tilde{x}}$ is identified at every value $V_{i}$ can take on, and is identified everywhere if $V_{i}$ has sufficiently large support.

Define $\tilde{u}_{i}(\tilde{x}) \equiv E\left(u_{i}\left(\tilde{X}, \epsilon_{i}^{*}\right) \mid \tilde{x}\right)=E\left(-\mathcal{S}_{i} \mid \tilde{x}\right)$. Then by definition the conditional mean of player $i$ 's baseline payoff function is $E\left[u_{i}^{*}\left(X, \epsilon_{i}^{*}\right) \mid x\right]=\alpha_{i} x_{i}+\tilde{u}_{i}(\tilde{x})$, and all other moments of the distribution of payoff functions likewise depend only on $\alpha_{i} x_{i}$ and on conditional moments of $\mathcal{S}_{i}$. So a goal is to estimate $\tilde{u}_{i}(\tilde{x})$, and more generally estimate conditional moments or quantiles of $\mathcal{S}_{i}$. Nonparametric estimators for conditional moments of $\mathcal{S}_{i}$ and their limiting distribution are provided by Lewbel, Linton, and McFadden (2011), except that they assumed an ordinary binary choice model where $V_{i}$ is observed (even determined by experimental design), whereas in our case $V_{i}$ is an estimated generated regressor, constructed from estimates of $\alpha_{i}, \delta_{i}(\tilde{x})$, and $p_{j}^{*}(x)$. We will later provide alternative estimators that take the estimation error in $V_{i}$ into account. Note that we cannot avoid this problem by taking the excluded regressor $x_{i}$ itself to be the special regressor because $x_{i}$ also appears in $p_{j}^{*}(x)$.

The rest of this section formalizes these constructions, and generalizes them to games with more than two players.

\subsection{Identifying $\alpha_{i}$ and $\delta_{i}$}

First consider identifying the marginal effects of excluded regressors $\left(\alpha_{i}\right)$, and the state-dependent component in interaction effects $\delta_{i}(\tilde{x})$. We begin by deriving the general game analog to equation (4).

For any $x$, let $\phi_{i}^{*}(x)$ denote the conditional expectation of $h_{i}\left(D_{-i}\right)$ given $x$, which is identifiable directly from data (with $h_{i}$ known to all $i$ and econometricians). When $h_{i}\left(D_{-i}\right)=\sum_{j \neq i} D_{j}$, $\phi_{i}^{*}(x) \equiv \sum_{j \neq i} p_{j}^{*}(x)$. For all $i, j$, let $\phi_{i, j}^{*}(x) \equiv \partial \phi_{i}^{*}(X) /\left.\partial X_{j}\right|_{X=x}$. Let $\mathcal{A}$ denote a $N$-vector with ordered coordinates $\left(\alpha_{1}, \alpha_{2}, . ., \alpha_{N}\right)$. For any $x$, define four $N$-vectors $\mathcal{W}_{1}(x), \mathcal{W}_{2}(x), \mathcal{V}_{1}(x), \mathcal{V}_{2}(x)$ whose ordered coordinates are $\left(p_{i, i}^{*}(x)\right)_{i \in N},\left(p_{1,2}^{*}(x), p_{2,3}^{*}(x), . ., p_{N-1, N}^{*}(x), p_{N, 1}^{*}(x)\right),\left(\phi_{i, i}^{*}(x)\right)_{i \in N}$ and $\left(\phi_{1,2}^{*}(x), \phi_{2,3}^{*}(x), . ., \phi_{N-1, N}^{*}(x), \phi_{N, 1}^{*}(x)\right)$ respectively. For any two vectors $R \equiv\left(r_{1}, r_{2}, . ., r_{N}\right)$ and $R^{\prime} \equiv\left(r_{1}^{\prime}, r_{2}^{\prime}, . ., r_{N}^{\prime}\right)$, let " $R . R^{\prime \prime}$ " and " $R . / R^{\prime \prime}$ " denote component-wise multiplication and divisions (i.e. $R . R^{\prime}=\left(r_{1} r_{1}^{\prime}, r_{2} r_{2}^{\prime}, . ., r_{N} r_{N}^{\prime}\right)$ and $\left.R . / R^{\prime}=\left(r_{1} / r_{1}^{\prime}, r_{2} / r_{2}^{\prime}, . ., r_{N} / r_{N}^{\prime}\right)\right)$.

Definition 1 A set $\omega$ satisfies the Non-degenerate and Non-singular (NDS) condition if it 
is in the interior of $\Omega_{X}$ and for all $x \in \omega$, (i) $0<p_{i}^{*}(x)<1$ for all $i$; and (ii) all coordinates in $\mathcal{W}_{2}(x), \mathcal{V}_{2}(x)$ and $\mathcal{V}_{2}(x) . \mathcal{W}_{1}(x)-\mathcal{V}_{1}(x) . \mathcal{W}_{2}(x)$ are non-zero.

The $N D S$ condition holds for $\omega$ when the support of $\mathcal{S}_{i}=-u_{i}\left(\tilde{x}, \epsilon_{i}^{*}\right)$ includes the support of $\alpha_{i} x_{i}+h_{i}\left(D_{-i}\right) \delta_{i}(\tilde{x})$ in its interior for any $x \equiv\left(x_{e}, \tilde{x}\right) \in \omega$. This happens when $x_{i}$ takes moderate values while $\epsilon_{i}^{*}$ varies sufficiently conditional on $\tilde{x}$ to generate a large support of $\mathcal{S}_{i}$. In any BNE and for any $x$,

$$
p_{i}^{*}(x)=F_{\mathcal{S}_{i} \mid \tilde{x}}\left(\alpha_{i} x_{i}+\delta_{i}(\tilde{x}) \phi_{i}^{*}(x)\right)
$$

for all $i \in N$. For any $\tilde{x}$, the smoothness of $F_{\epsilon^{*} \mid \tilde{x}}$ in $\mathrm{A} 1$ and the continuity of $u_{i}$ in $\epsilon_{i}^{*}$ given $\tilde{x}$ in A3 imply partial derivatives of $p_{i}^{*}, \phi_{i}^{*}$ with respect to excluded regressors exist over $\omega$. To have all coordinates in $\mathcal{W}_{2}(x), \mathcal{V}_{2}(x)$ and $\mathcal{V}_{2}(x) . \mathcal{W}_{1}(x)-\mathcal{V}_{1}(x) \cdot \mathcal{W}_{2}(x)$ be non-zero as NDS requires, $\delta_{i}(\tilde{x})$ must necessarily be non-zero for all $i$. This rules out uninteresting cases with no strategic interaction between players. ${ }^{7}$

Theorem 1 Suppose A1-3 hold and $\omega$ satisfies NDS. Then (i) $\left(\alpha_{1}, ., \alpha_{N}\right)$ are identified as signs of the ordered coordinates of

$$
E\left[\left(\mathcal{W}_{1}(X)-\mathcal{W}_{2}(X) \cdot \mathcal{V}_{1}(X) \cdot / \mathcal{V}_{2}(X)\right) 1\{X \in \omega\}\right]
$$

(ii) For any $\tilde{x}$ such that $\left\{x_{e}:\left(x_{e}, \tilde{x}\right) \in \omega\right\} \neq \varnothing$, the vector $\left(\delta_{1}(\tilde{x}), ., \delta_{N}(\tilde{x})\right)$ is identified as:

$$
\mathcal{A} . E\left[\mathcal{W}_{2}(X) \cdot /\left(\mathcal{V}_{2}(X) \cdot \mathcal{W}_{1}(X)-\mathcal{V}_{1}(X) \cdot \mathcal{W}_{2}(X)\right) \mid X \in \omega, \tilde{X}=\tilde{x}\right]
$$

Remark 1.1 For the special case of $N=2$ with with $h_{1}\left(D_{-1}\right)=D_{2}$ and $h_{2}\left(D_{-2}\right)=D_{1}$, this theorem is simplified to the identification argument described in the previous subsection.

Remark 1.2 Our identification of $\left(\alpha_{i}\right)_{i \in N}$ in Theorem 1 does not depend on identification at infinity (i.e. does not need to send the excluded regressor $x_{i}$ to positive or negative infinity in order to recover the sign of $\alpha_{i}$ from the limit of $i$ 's choice probabilities). Thus our argument does not hinge on observing states that are far out in the tails of the distribution. The same is true for identification of $\left(\delta_{i}(\tilde{x})\right)_{i \in N}$.

Remark 1.3 If $\left(\delta_{i}(\tilde{x})\right)_{i \in N}$ consists of a vector of constants (i.e. $\delta_{i}(\tilde{x})=\delta_{i}$ for all $\left.i, \tilde{x}\right)$, then $\left(\delta_{1}, ., \delta_{N}\right)$ are identified as $\mathcal{A} . E\left[\mathcal{W}_{2}(x) . /\left(\mathcal{V}_{2}(x) . \mathcal{W}_{1}(x)-\mathcal{V}_{1}(x) . \mathcal{W}_{2}(x)\right) \mid X \in \omega\right]$ by pooling data from all games with states in $\omega$. In the earlier two person game this reduces to

$$
\delta_{1}=\alpha_{1} E\left[\left(p_{1,1}^{*}(X) p_{2,2}^{*}(X)-p_{2,1}^{*}(X) p_{1,2}^{*}(X)\right)^{-1} p_{1,2}^{*}(X) \mid X \in \omega\right] .
$$

\footnotetext{
${ }^{7}$ To see this, note if $\delta_{i}(\tilde{x})=0$ for some $i$, then $p_{i}^{*}(x)$ would be independent from $X_{j}$ for all $j \neq i$ in any BNE and some of the diagonal entries in $\mathcal{W}_{2}$ (and $\mathcal{V}_{2}$ ) would be zero for any $x$. Other than this, the NDS condition not restrict how the non-excluded states enter $\delta_{i}(\tilde{x})$ at all. The condition that all diagonal entries in $\mathcal{V}_{2} . \mathcal{W}_{1}-\mathcal{V}_{1} . \mathcal{W}_{2}$ be non-zero can be verified from data, because these entries only involve functions that are directly identifiable.
} 
and similarly for $\delta_{2}$.

Remark 1.4 With more than two players, there exists additional information that can be used to identify $\alpha_{i}$ and $\delta_{i}$, based on using different partial derivatives from those in $\mathcal{W}_{2}$ and $\mathcal{V}_{2}$. For example, the proof can be modified to replace $\mathcal{W}_{2}$ with $\left(p_{1, N}^{*}, p_{2,1}^{*}, p_{3,2}^{*}, \ldots, p_{N-1, N-2}^{*}, p_{N, N-1}^{*}\right)$ and $\mathcal{V}_{2}$ defined accordingly. This is an additional source of over-identification that might be exploited to improve efficiency in estimation.

\subsection{Recovering distributions of $\mathcal{S}_{i}$ given non-excluded regressors}

For any $i$ and $x \equiv\left(x_{e}, \tilde{x}\right)$, our "generated special regressor" is defined as:

$$
V_{i}\left(x ; \alpha_{i}, \delta_{i}, \phi_{i}^{*}\right) \equiv \alpha_{i} x_{i}+\delta_{i}(\tilde{x}) \phi_{i}^{*}(x)
$$

For any $\tilde{x}$ with $\left(\delta_{i}(\tilde{x})\right)_{i \in N}$ identified, such a generated regressor can be constructed from the distribution of actions and states observed from data. Denote the support of $V_{i}$ conditional on $\tilde{X}=\tilde{x}$ by $\Omega_{V_{i} \mid \tilde{x}}$. That is, $\Omega_{V_{i} \mid \tilde{x}} \equiv\left\{t \in \mathbb{R}: \exists x_{e} \in \Omega_{X_{e} \mid \tilde{x}}\right.$ s.t. $\left.\alpha_{i} x_{i}+\delta_{i}(\tilde{x}) \phi_{i}^{*}(x)=t\right\}$.

Theorem 2 Suppose A1-3 hold and $\alpha_{i}$ and $\delta_{i}(\tilde{x})$ are both identified at $\tilde{x}$. Then $F_{\mathcal{S}_{i} \mid \tilde{x}}$ is identified over $\Omega_{V_{i} \mid \tilde{x}}$.

To prove Theorem 2, observe that for a fixed $\tilde{x}$, A3 guarantees that variation in $X_{e}$ does not affect the distribution of $\mathcal{S}_{i}$ given $\tilde{x}$. Given the unique BNE in data from A2, the choice probabilities identified from data can be related to the distribution of $\mathcal{S}_{i}$ given $\tilde{x}$ as:

$$
\operatorname{Pr}\left(D_{i}=1 \mid X=\left(x_{e}, \tilde{x}\right)\right)=\operatorname{Pr}\left(\mathcal{S}_{i} \leq V_{i}\left(x_{e}, \tilde{x}\right) \mid \tilde{X}=\tilde{x}\right)
$$

for all $i$ (where the equality follows from independence between $X_{e}$ and $\epsilon_{i}^{*}$ given $\tilde{x}$ in A3). Because the left-hand side of (10) is directly identifiable, we can recover $F_{\mathcal{S}_{i} \mid \tilde{x}}(t)$ for all $t$ on the support of $V_{i}(X)$ given $\tilde{x}$. It also follows that the conditional quantiles of $u_{i}\left(\tilde{X}, \epsilon_{i}^{*}\right)$ given $\tilde{X}=\tilde{x}$ can also be identified as $\inf \left\{t: \operatorname{Pr}\left(-\mathcal{S}_{i} \leq t \mid \tilde{x}\right) \geq \alpha\right\}$ for all $\alpha \in\left\{t \in(0,1): \exists x\right.$ such that $\operatorname{Pr}\left(D_{i}=1 \mid x\right)=$ $t\}$. Since $\alpha_{i}$ is identified, identification of $F_{\mathcal{S}_{i} \mid \tilde{x}}$ implies identification of the conditional distribution of baseline probabilities $u_{i}^{*}\left(x, \epsilon_{i}^{*}\right)=\alpha_{i} x_{i}+u_{i}\left(\tilde{x}, \epsilon_{i}^{*}\right)$ given $x$.

\subsection{Identifying $u_{i}$ under mean independence}

A4 (Reparametrization) For all $i, E\left[u_{i}\left(\tilde{X}, \epsilon_{i}^{*}\right) \mid \tilde{x}\right]$ is bounded for all $\tilde{x}$, so that $u_{i}\left(\tilde{X}, \epsilon_{i}^{*}\right)$ can be reparametrized as $\tilde{u}_{i}(\tilde{X})-\epsilon_{i}$, with $\epsilon_{i} \equiv \tilde{u}_{i}(\tilde{X})-u_{i}\left(\tilde{X}, \epsilon_{i}^{*}\right)$ and $\tilde{u}_{i}(\tilde{X}) \equiv E\left[u_{i}\left(\tilde{X}, \epsilon_{i}^{*}\right) \mid \tilde{X}\right]$. 
Note that A4 is a free location normalization provided the boundedness condition holds. By construction, $E\left[\epsilon_{i} \mid \tilde{x}\right]=0$ for all $i$ and $\tilde{x}$; and the conditional distribution $F_{\epsilon \mid X}$ satisfies previous restrictions on $F_{\epsilon^{*} \mid X}$ (that is, A1 and condition (ii) in A3 holds with $\epsilon^{*}$ replaced by $\epsilon$ ).

A5 (Large Support and Positive Density) For any $i$ and $\tilde{x}$, (i) the support of $V_{i}(X)$ given $\tilde{x}$, denoted $\Omega_{V_{i} \mid \tilde{x}}$, includes the support of $\mathcal{S}_{i}$ given $\tilde{x}$; and (ii) the density of $V_{i}(X)$ given $\tilde{x}$ is positive almost surely w.r.t. Lebesgue Measure over $\Omega_{V_{i} \mid \tilde{x}}$.

The next corollary establishes the identification of $\tilde{u}_{i}(\tilde{x})$, and hence of the conditional mean of baseline payoffs. The "large support" assumption (condition (i)) in A5 is key for identification. This is analogous to the large support condition for special regressors in single-agent models in Lewbel (2000). In the special case of the two player game considered earlier, A5-(i) holds if the support of $X_{i}$ given $\tilde{x}$ is large enough relative to $\delta_{i}(\tilde{x})$ and the support of $\epsilon_{i}$ given $\tilde{x}$. We include detailed primitive conditions that are sufficient for A5 in Appendix B. Also note the large support condition A5-(i) can be checked using data. Specifically, $\Omega_{\mathcal{S}_{i} \mid \tilde{x}} \subseteq \Omega_{V_{i} \mid \tilde{x}}$ if and only if the supreme and the infimum of $\operatorname{Pr}\left(D_{i}=1 \mid V_{i}=v, \tilde{X}=\tilde{x}\right)$ over $v \in \Omega_{V_{i} \mid \tilde{x}}$ are respectively 1 and 0 .

Corollary 1 (Theorem 2) Under A1-4 and 5(i), $\tilde{u}_{i}(\tilde{x})$ is identified as $E\left[-\mathcal{S}_{i} \mid \tilde{x}\right]$.

For a given $\tilde{x}$, Theorem 2 implies we can recover the distribution $F_{\mathcal{S}_{i} \mid \tilde{x}}$ over its full support, as long as the support of the generated regressor $V_{i}$ given $\tilde{x}$ covers the support of $\mathcal{S}_{i}$ given $\tilde{x}$. This then suffices to identify $\tilde{u}_{i}(\tilde{x})$ since $\tilde{u}_{i}(\tilde{x})=E\left[-\mathcal{S}_{i} \mid \tilde{x}\right]$. Corollary 1 then follows immediately given that the distribution of $\mathcal{S}_{i}$ given $\tilde{x}$ implies the identification of $E\left[-\mathcal{S}_{i} \mid \tilde{x}\right]$.

With $\alpha_{i}, \delta_{i}(\tilde{x})$ and $\tilde{u}_{i}(\tilde{x})$ identified at $\tilde{x}$, the distribution of $\epsilon_{i}$ given $\tilde{x}$ is also identified, and can be recovered as

$$
\begin{aligned}
& E\left(D_{i} \mid V_{i}=v, \tilde{X}=\tilde{x}\right)=\operatorname{Pr}\left(\epsilon_{i} \leq \tilde{u}_{i}(\tilde{X})+V_{i} \mid v, \tilde{x}\right)=F_{\epsilon_{i} \mid \tilde{x}}\left(\tilde{u}_{i}(\tilde{x})+v\right) \\
\Longrightarrow & F_{\epsilon_{i} \mid \tilde{x}}(e)=E\left[D_{i} \mid V_{i}=e-\tilde{u}_{i}(\tilde{x}), \tilde{X}=\tilde{x}\right]
\end{aligned}
$$

for all $e$ on the support $\Omega_{\epsilon_{i} \mid \tilde{x}}$. By A5, the support of $V_{i}$ given $\tilde{x}$ is sufficiently large to ensure that $F_{\epsilon_{i} \mid \tilde{x}}(e)$ is identified for all $e$ on the support $\Omega_{\epsilon_{i} \mid \tilde{x}}$. Thus it is possible for econometricians to predict equilibrium outcomes under known counterfactual changes of the error distribution.

The remainder of this subsection introduces a useful shortcut for constructing $\tilde{u}_{i}(\tilde{x})$ from observable distributions. Such a shortcut allows us to back out $\tilde{u}_{i}(\tilde{x})$ without having to recover the whole distribution $F_{\mathcal{S}_{i} \mid \tilde{x}}$ under the mild conditions of positive densities in A5-(ii). Let $c^{*}$ be a constant that lies in the interior of $\Omega_{V_{i} \mid \tilde{x}}$. For all $i$ and $x=\left(x_{e}, \tilde{x}\right)$ define

$$
Y_{i}^{*}\left(d_{i}, x\right) \equiv \frac{d_{i}-1\left\{V_{i}(x) \geq c^{*}\right\}}{f_{V_{i} \mid \tilde{x}}\left(V_{i}(x)\right)}
$$


The choice of $c^{*}$ is feasible in estimation, for the support $\Omega_{V_{i} \mid \tilde{x}}$ is known as long as $\alpha_{i}, \delta_{i}(\tilde{x})$ are identified. Such a choice of $c^{*}$ is also allowed to depend on $\tilde{x}$.

Theorem 3 Suppose A1-5 hold, and $\alpha_{i}$ and $\delta_{i}(\tilde{x})$ are both identified at $\tilde{x}$. Then:

$$
\tilde{u}_{i}(\tilde{x})=E\left[Y_{i}^{*} \mid \tilde{X}=\tilde{x}\right]-c^{*}
$$

for any $c^{*}$ in the interior of $\Omega_{V_{i} \mid \tilde{x}}$.

Proof of Theorem 3. For all $i$ and $\tilde{x}$,

$$
\begin{aligned}
& E\left(Y_{i}^{*} \mid \tilde{x}\right)=E\left[E\left(Y_{i}^{*} \mid V_{i}, \tilde{X}\right) \mid \tilde{X}=\tilde{x}\right]=\int_{\Omega_{V_{i} \mid \tilde{x}}} \frac{E\left[D_{i} \mid v_{i}, \tilde{x}\right]-1\left\{v_{i}>c^{*}\right\}}{f_{V_{i}}\left(v_{i} \mid \tilde{x}\right)} f_{V_{i}}\left(v_{i} \mid \tilde{x}\right) d v_{i} \\
= & \int_{\Omega_{V_{i} \mid \tilde{x}}} E\left[1\left\{\epsilon_{i} \leq v_{i}+\tilde{u}_{i}(\tilde{x})\right\}-1\left\{v_{i}>c^{*}\right\} \mid v_{i}, \tilde{x}\right] d v_{i} \\
= & \int_{\Omega_{V_{i} \mid \tilde{x}}} \int_{\Omega_{\epsilon_{i} \mid \tilde{x}}} 1\left\{\varepsilon_{i} \leq v_{i}+\tilde{u}_{i}(\tilde{x})\right\}-1\left\{v_{i}>c^{*}\right\} d F_{\epsilon_{i}}\left(\varepsilon_{i} \mid \tilde{x}\right) d v_{i} \\
= & \int_{\Omega_{\epsilon_{i} \mid \tilde{x}}} \int_{\Omega_{V_{i} \mid \tilde{x}}} 1\left\{v_{i} \geq s_{i}\right\}-1\left\{v_{i}>c^{*}\right\} d v_{i} d F_{\epsilon_{i}}\left(\varepsilon_{i} \mid \tilde{x}\right)
\end{aligned}
$$

where $s_{i}$ is a shorthand for $-\tilde{u}_{i}(\tilde{x})+\varepsilon_{i}$. The first equality follows from the Law of Iterated Expectation, and the second and the third from the definitions of $Y_{i}^{*}, V_{i}$ and positive densities in A5-(ii) respectively. The fourth follows from the independence between $\epsilon_{i}$ and $X_{e}$ given $\tilde{x}$, which implies $F_{\epsilon_{i}}\left(. \mid v_{i}, \tilde{x}\right)=F_{\epsilon_{i}}(. \mid \tilde{x})$ for all $v_{i}, \tilde{x}$. The last equality follows from a change of the orders of integration, which is due to the Fubini's Theorem. The last expression on the right-hand side of (12) can be written as

$$
\begin{aligned}
& \int_{\Omega_{\epsilon_{i} \mid \tilde{x}}} \int_{\Omega_{V_{i} \mid \tilde{x}}} 1\left\{s_{i} \leq v_{i}<c^{*}\right\} 1\left\{s_{i} \leq c^{*}\right\}-1\left\{s_{i} \geq v_{i}>c^{*}\right\} 1\left\{s_{i}>c^{*}\right\} d v_{i} d F_{\epsilon_{i}}\left(\varepsilon_{i} \mid \tilde{x}\right) \\
= & \int_{\Omega_{\epsilon_{i} \mid \tilde{x}}}\left(1\left\{s_{i} \leq c^{*}\right\} \int_{s_{i}}^{c^{*}} d v_{i}-1\left\{s_{i}>c^{*}\right\} \int_{c^{*}}^{s_{i}} d v_{i}\right) d F_{\epsilon_{i}}\left(\varepsilon_{i} \mid \tilde{x}\right)=\int_{\Omega_{\epsilon_{i} \mid \tilde{x}}}\left(c^{*}-s_{i}\right) d F_{\epsilon_{i}}\left(\varepsilon_{i} \mid \tilde{x}\right) \\
= & c^{*}+\int_{\Omega_{\epsilon_{i} \mid \tilde{x}}}\left(\tilde{u}_{i}(\tilde{x})-\varepsilon_{i}\right) d F_{\epsilon_{i}}\left(\varepsilon_{i} \mid \tilde{x}\right)=c^{*}+\tilde{u}_{i}(\tilde{x})
\end{aligned}
$$

where the second equality uses the large support condition in A5-(i). Hence $E\left[Y_{i}^{*} \mid \tilde{X}=\tilde{x}\right]-c^{*}=$ $\tilde{u}_{i}(\tilde{x})$ Q.E.D.

\section{Fixed Cost Components as Excluded Regressors in Entry Games}

In this section, we show how some components in firms' fixed costs in classical entry games satisfy properties required to be excluded regressors in our model. We also provide a context for economic interpretation of these properties. 
Consider a two stage entry game with two players $i \in\{1,2\}$. When we refer to one player as $i$, the other will be denoted $j$. In the first stage of the game firms decide whether to enter. In the second stage each player observes the other's entry decision and hence knows the market structure (monopoly or duopoly). If duopoly occurs, then in the second stage firms engage in Cournot competition, choosing output quantities. The inverse market demand function face by firm $i$ is denoted $\psi^{M}\left(q_{i} ; \tilde{x}\right)$ if there is monopoly, and $\psi^{D}\left(q_{i}, q_{j} ; \tilde{x}\right)$ if there is duopoly, where $q_{i}$ denotes quantities produced by $i$ and $\tilde{x}$ a vector of market- or firm-level characteristics observed by both firms and the econometrician. That the inverse market demand functions only depend on the commonly observed $\tilde{x}$ reflects the assumption that researchers can obtain the same information about commonly observed state variables as consumers in the market.

Costs for firms are given by $c_{i}\left(q_{i} ; \tilde{x}\right)+x_{i}+\varepsilon_{i}$ for $i \in\{1,2\}$, where the function $c_{i}$ specifies $i$ 's variable costs, the scalar $x_{i}$ is a commonly observed component of $i$ 's fixed cost, and $\varepsilon_{i}$ is a component of $i$ 's fixed cost that is privately observed by $i$. The fixed costs $x_{i}+\varepsilon_{i}$ (and any other fixed costs that may be absorbed in $c_{i}$ function) are paid after entry but before the choice of production quantities. Both $\left(x_{1}, x_{2}\right)$ and $\tilde{x}$ are known to both firms as they make entry decisions in the first stage, and can be observed by researchers in data. A firm that decides not to enter receives zero profit in the second stage.

If only firm $i$ enters in the first stage, then its profit is given by $\psi^{M}\left(q^{*} ; \tilde{x}\right) q^{*}-c_{i}\left(q^{*} ; \tilde{x}\right)-x_{i}-$ $\varepsilon_{i}$, where $q^{*}$ solves

$$
\frac{\partial}{\partial q} \psi^{M}\left(q^{*} ; \tilde{x}\right) q^{*}+\psi^{M}\left(q^{*} ; \tilde{x}\right)=\frac{\partial}{\partial q} c_{i}\left(q^{*} ; \tilde{x}\right)
$$

assuming an interior solution exists. If there is a duopoly in the second stage, then $i$ 's profit is $\psi^{D}\left(q_{i}^{*}, q_{j}^{*}\right) q_{i}^{*}-c_{i}\left(q_{i}^{*} ; \tilde{x}\right)-x_{i}-\varepsilon_{i}$ where $\left(q_{i}^{*}, q_{j}^{*}\right)$ solves

$$
\begin{aligned}
\frac{\partial}{\partial q_{i}} \psi^{D}\left(q_{i}^{*}, q_{j}^{*} ; \tilde{x}\right) q_{i}+\psi^{D}\left(q_{i}^{*}, q_{j}^{*} ; \tilde{x}\right) & =\frac{\partial}{\partial q_{i}} c_{i}\left(q_{i}^{*} ; \tilde{x}\right) \text { and } \\
\frac{\partial}{\partial q_{j}} \psi^{D}\left(q_{i}^{*}, q_{j}^{*} ; \tilde{x}\right) q_{j}+\psi^{D}\left(q_{i}^{*}, q_{j}^{*} ; \tilde{x}\right) & =\frac{\partial}{\partial q_{j}} c_{j}\left(q_{j}^{*} ; \tilde{x}\right),
\end{aligned}
$$

assuming interior solutions exist. The non-entrant's profit is 0 . The entrants' choices of outputs are functions of $\tilde{x}$ alone in both cases. This is because by definition both the commonly observed and the idiosyncratic (and privately known) component of the fixed costs $\left(x_{i}\right.$ and $\left.\varepsilon_{i}\right)$ do not vary with output. Hence an entrant's profit under either structure takes the form $\pi_{i}^{s}(\tilde{x})-x_{i}-\varepsilon_{i}$, with $s \in\{D, M\}$, with $\pi_{i}^{s}$ being the difference between firm $i$ 's revenues and variable costs under market structure $s$.

In the first stage, firms simultaneously decide whether to enter or not, based on a calculation of the expected profit from entry conditional on the public information $X \equiv\left(\tilde{X}, X_{1}, X_{2}\right)$ and private information $\varepsilon_{i}$. The following matrix summarizes profits in all scenarios, taking into account firms' Cournot Nash strategies under duopoly and profit maximization under monopoly (Firm 1 is the 
row player, whose profit is the first entry in each cell):

\begin{tabular}{|c|c|c|}
\hline & Enter & Stay out \\
\hline \hline Enter & $\left(\pi_{1}^{D}(\tilde{X})-X_{1}-\epsilon_{1}, \pi_{2}^{D}(\tilde{X})-X_{2}-\epsilon_{2}\right)$ & $\left(\pi_{1}^{M}(\tilde{X})-X_{1}-\epsilon_{1}, 0\right)$ \\
\hline Stay out & $\left(0, \pi_{2}^{M}(\tilde{X})-X_{2}-\epsilon_{2}\right)$ & $(0,0)$ \\
\hline
\end{tabular}

It follows that $i$ 's expected profits from entry are:

$$
\pi_{i}^{M}(\tilde{x})+p_{j}(x) \delta_{i}(\tilde{x})-x_{i}-\varepsilon_{i}
$$

where $\delta_{i}(\tilde{x}) \equiv \pi_{i}^{D}(\tilde{x})-\pi_{i}^{M}(\tilde{x})$, and $p_{j}(x)$ is the probability that firm $j$ decides to enter conditional on $i$ 's information set $\left(x, \varepsilon_{i}\right)$. That $p_{j}$ does not depend on $\varepsilon_{i}$ is due to the assumed independence between private information conditional on $\tilde{x}$ in A1. The expected profits from staying out is zero. Hence, in any Bayesian Nash equilibrium, $i$ decides to enter if and only if the expression in (13) is greater than zero, and $\operatorname{Pr}\left(D_{1}=d_{1}, D_{2}=d_{2} \mid x\right)=\Pi_{i=1,2} p_{i}(x)^{d_{i}}\left(1-p_{i}(x)\right)^{1-d_{i}}$, where

$$
p_{i}(x)=\operatorname{Pr}\left\{\epsilon_{i} \leq \pi_{i}^{M}(\tilde{x})+p_{j}(x) \delta_{i}(\tilde{x})-x_{i} \mid x\right\}
$$

for $i=1,2$. This fits in the class of games considered in the paper, with $V_{i}(X) \equiv p_{j}(X) \delta_{i}(\tilde{X})-X_{i}$ being the generated special regressor.

Examples of fixed cost components $\left(x_{1}, x_{2}\right)$ that could be public knowledge include lump-sum expenses such as equipment costs, maintenance fees and patent/license fees, while $\left(\epsilon_{1}, \epsilon_{2}\right)$ could be idiosyncratic administrative overhead such as firms' office rental or supplies. The non-excluded regressors $\tilde{x}$ would include market or industry level demand and cost shifters. In these examples the fixed cost components $\left(\epsilon_{1}, \epsilon_{2}\right)$ and $\left(X_{1}, X_{2}\right)$ are incurred by different aspects of the production process, making it plausible that they are independent of each other (after conditioning on the given market and industry conditions $\tilde{x}$ ), thereby satisfying the conditional independence in A3.

The large support assumption required for identifying $\tilde{u}_{i}(\tilde{x})$ in Section 3.4 also holds, as long as the support for publicly known fixed costs is large relative to that of privately known fixed cost components. For example, a set of sufficient (but not necessary) conditions for the large support assumption to hold at a given $\tilde{x}$ are: (a) the support of $\epsilon_{i}$ given $\tilde{x}$ is included in $[0, \bar{\varepsilon}]$ with $\bar{\varepsilon} \leq \pi_{i}^{M}(\tilde{x})$; (b) there exists $\left(x_{i}, x_{j}\right)$ with $p_{j}\left(x_{i}, x_{j}, \tilde{x}\right)=0$ and $\pi_{i}^{M}(\tilde{x})-\bar{\varepsilon} \geq x_{i}$; and (c) there exists $\left(\hat{x}_{i}, \hat{x}_{j}\right)$ with $p_{j}\left(\hat{x}_{i}, \hat{x}_{j}, \tilde{x}\right)=1$ and $\pi_{i}^{D}(\tilde{x}) \leq x_{i}$. In economic terms, (a) means monopoly profits are sufficiently high before subtracting commonly known fixed costs $X_{i}$; while (b) and (c) require that $x_{j}$ can be relatively high for firm $j$ while $x_{i}$ is relatively low for firm $i$; and vice versa. ${ }^{8}$

More generally, the large support assumption has an immediate economic interpretation. It means that, conditional on commonly observed demand shifters (non-excluded states in $\tilde{X}$ ), a firm

\footnotetext{
${ }^{8} \mathrm{We}$ are grateful to an anonymous referee for pointing this out.
} 
$i$ may not find it profitable to enter even when private fixed costs $\epsilon_{i}$ are as low as possible. This can happen if $X_{i}$ (the publicly known component of fixed cost) is too high, or the competition is too intense (in the sense that the other firm's equilibrium entry probability $p_{j}$ is too high). Large support also means that firm $i$ could decide to enter even when $\epsilon_{i}$ is as high as maximum possible, because $X_{i}$ and $p_{j}(X)$ can be sufficiently low while the market demand is sufficiently high to make entry attractive. These assumptions on the supports of $X_{i}$ and $\epsilon_{i}$ are particularly plausible in applications where publicly known components of fixed costs are relatively large, e.g., the fixed costs in acquiring land, buildings, and equipment and acquiring license/patents could be much more substantial than those incurred by idiosyncratic supplies and overhead. Finally, note that the economic implications of the large support condition can in principle be verified by checking whether firms' entry probabilities are degenerate at 0 and 1 for sufficiently high or low levels of the publicly known components of fixed costs.

\section{$5 \quad$ A Simplified Estimator for $\tilde{u}_{i}$}

Estimation for payoffs could be done in principle using sample analogs of Theorem 3. Nonetheless we introduce a refined and simplified argument for identifying $\tilde{u}_{i}$ in this subsection, in order to simplify the limiting distribution theory for semiparametric estimators. We then build on a sample analog of the approach in this subsection to construct a relatively easier estimator in Section 6, and

establish its limiting distribution. For any $i \in N$, let $x_{e,-i}$ denote $\left(x_{j}\right)_{j \in N \backslash\{i\}}$ and let $x_{-i}$ denote $\left(x_{e,-i}, \tilde{x}\right)$.

A5' For any $i$ and $\tilde{x}$, there exists some $x_{-i}$ such that (i) the support of $V_{i}$ given $x_{-i}$ includes the support of $\mathcal{S}_{i}$ given $\tilde{x}$; (ii) the conditional density of $X_{i}$ given $x_{-i}$ exists and is positive almost everywhere over its full support; and (iii) the sign of $\partial V_{i}(X) /\left.\partial X_{i}\right|_{X=\left(x_{i}, x_{-i}\right)}$ equals the sign of $\alpha_{i}$ for all $x_{i} \in \Omega_{X_{i} \mid x_{-i}}$.

The large support condition in A5' is stronger than that in A5. To see this, note the support of $F_{\mathcal{S}_{i} \mid x}$ does not vary with $x_{e}$ under the conditional independence of excluded regressors in A3. While condition (i) in A5 and A5' both require generated special regressors to vary sufficiently and cover the same support of $F_{\mathcal{S}_{i} \mid \tilde{x}}$, the latter is more stringent in that it requires such variation be generated only by variation in $X_{i}$ while $x_{-i}$ is fixed. In comparison, the former fixes $\tilde{x}$ and allow variations in $V_{i}$ to be generated by all excluded regressors in $X_{e}$. This stronger support condition (i) in A5' also provides a source of over-identification of $\tilde{u}_{i}(\tilde{x})$. Similar to A5, the large support condition in A5' can in principle be checked using observable distributions. That is, $\Omega_{\mathcal{S}_{i} \mid \tilde{x}} \subseteq \Omega_{V_{i} \mid x_{-i}}$ if and only if the supreme and infimum of $\operatorname{Pr}\left(D_{i}=1 \mid X_{i}=t, X_{-i}=x_{-i}\right)$ over $t \in \Omega_{X_{i} \mid x_{-i}}$ are 1 and 0 respectively.

The last condition in A5' requires the generated special regressor associated with $i$ to be 
monotone in the excluded regressor $X_{i}$ given $\left(x_{e,-i}, \tilde{x}\right)$. It holds when $X_{i}$ 's direct marginal effect on $i$ 's latent utility (i.e. $\alpha_{i}$ ) is not offset by its indirect marginal effect (i.e. $\delta_{i}(\tilde{x}) \phi_{i, i}^{*}\left(x_{i}, x_{-i}\right)$ ) for all $x_{i} \in \Omega_{X_{i} \mid x_{-}}$. This condition is needed for the alternative method for recovering $\tilde{u}_{i}$ below (Corollary 2), which builds on changing variables between excluded regressors and generated special regressors. Such a monotonicity condition is not needed for the identification argument in Section 3.4 (Theorem 3).

Condition (iii) in A5' holds under several scenarios with mild conditions. One such scenario is when $x_{e,-i}$ takes extreme values. To see this, consider the case with $N=2$ and $h_{i}\left(D_{-i}\right)=D_{j}$. The marginal effect of $X_{i}$ on $V_{i}$ at $x=\left(x_{i}, x_{j}, \tilde{x}\right)$ is $\alpha_{i}+\delta_{i}(\tilde{x}) \partial p_{j}^{*}(X) /\left.\partial X_{i}\right|_{X=x}$, where $\alpha_{i}$ and $\delta_{i}(\tilde{x})$ are both finite constants given $\tilde{x}$. With $x_{j}$ taking extremely large or small values, the impact of $x_{i}$ on $p_{j}^{*}$ in BNE gets close to 0 . Thus the sign of $\alpha_{i}+\delta_{i}(\tilde{x}) p_{j, i}^{*}(x)$ will be identical to the sign of $\alpha_{i}$ over $\Omega_{X_{i} \mid x_{-i}}$ for such $x_{j}$ and $\tilde{x}$. In another scenario, A5'-(iii) can even hold uniformly over the entire support of $X$, provided both $\delta_{i}(\tilde{x})$ and the conditional density of players' private information $f_{\epsilon_{i} \mid \tilde{x}}$ are bounded above by small constants. In Appendix B, we formalize in details these sufficient primitive conditions for the monotonicity in A5'-(iii).

We now show how conditions in A5' can be used to provide a short-cut for identifying $\tilde{u}_{i}(\tilde{x})$. Fix $i$ and $\tilde{x}$, and consider any $x_{e,-i}$ that satisfies A5' for $i$ and $\tilde{x}$. Let $\underline{v_{i}}\left(x_{-i}\right)$ and $\overline{v_{i}}\left(x_{-i}\right) \in \mathbb{R} \cup\{-\infty,+\infty\}$ denote the infimum and the supremum of the conditional support $\Omega_{V_{i} \mid x_{-i}}$. Let $H$ be a continuous cumulative distribution (to be chosen by the econometrician) with a bounded interval support that is a subset of $\Omega_{V_{i} \mid x_{-i}}$. The choice of $H$ is allowed to depend on both $i$ and $x_{-i}$, though for simplicity we will suppress any such dependence in notation. Choosing such a distribution $H$ with the required support is feasible in estimation, since $\alpha_{i}$ and $\delta_{i}(\tilde{x})$ (and consequently conditional support $\Omega_{V_{i} \mid \tilde{x}}$ is identified. Let $\mu_{H}$ denote the expectation of $H$, which is known because $H$ is chosen by the econometrician, and which could potentially depend on $x_{-i}$. We discuss how to choose $H$ in estimation in Section 6.2. For any $i$ and $x$ define

$$
Y_{i, H} \equiv \frac{\left[d_{i}-H\left(V_{i}(x)\right)\right]\left[1+\alpha_{i} \delta_{i}(\tilde{x}) \phi_{i, i}^{*}(x)\right]}{f_{X_{i}}\left(x_{i} \mid x_{-i}\right)}
$$

where $\alpha_{i}+\delta_{i}(\tilde{x}) \phi_{i, i}^{*}(x)$ by construction is the marginal effect of the excluded regressor $x_{i}$ on the generated special regressor $V_{i}(x)$. Note $Y_{i, H}$ is well-defined under A5', for $f_{X_{i} \mid x_{-i}}$ is positive almost everywhere over $\Omega_{X_{i} \mid x_{-i}}$.

Corollary 2 (Theorem 3) Suppose A1-4 hold, and both $\alpha_{i}$ and $\delta_{i}(\tilde{x})$ are identified at $\tilde{x}$. For any $i$ and $x_{-i}=\left(x_{e,-i}, \tilde{x}\right)$ such that A5' holds,

$$
\tilde{u}_{i}(\tilde{x})=E\left[Y_{i, H} \mid x_{-i}\right]-\mu_{H} .
$$

Remark 4.1 If zero is in the support of $V_{i}$ given $x_{-i}$ then Corollary 2 holds taking $H$ to be the degenerate distribution function $H(V)=I\{V \geq 0\}$ and $\mu_{H}=0$. Corollary 2 generalizes the special 
regressor results in Lewbel (2000) and Lewbel, Linton, and McFadden (2011) by allowing $H($.$) to$ be an arbitrary distribution function instead of a degenerate distribution function, and by using the density of an excluded regressor rather than that of the special regressor in the denominator of the endogenous variable constructed. Both modifications simplify the limiting distribution theory in our context where the special regressor is also a generated regressor. In particular, choosing $H$ to be continuously differentiable allows us to take standard Taylor expansions to linearize the estimator as a function of the estimated $V_{i}$ around the true $V_{i}$. Dividing by the density of $X_{i}$ instead of the density of $V_{i}$ requires estimating the density of an observed regressor instead of the estimating the density of an estimated regressor.

Remark 4.2 It is clear from Corollary 2 that $\tilde{u}_{i}(\tilde{x})$ can be recovered using any $(N-1)$-vector $x_{e,-i}$ such that the support of $V_{i}$ given $x_{-i}=\left(x_{e,-i}, \tilde{x}\right)$ is large enough and $V_{i}(X)$ is monotone in $x_{i}$ given $x_{-i}$. As such, $\tilde{u}_{i}(\tilde{x})$ is over-identified. The source of over-identification is due to the stronger support condition required in condition (i) of A5'.

Remark 4.3 The large support condition (i) and the monotonicity condition (iii) in A5' could be checked from observable distribution. This is because $\operatorname{Pr}\left(D_{i}=1 \mid V_{i}=v, x_{-i}\right)$ is identified over $v \in \Omega_{V_{i} \mid x_{-i}}$ for any $x_{-i}$, and the marginal impact of excluded regressors on generated special regressors are identified.

\section{Semiparametric Estimation of Index Payoffs}

We estimate a two-player semi-parametric model, where players have constant interaction effects and index payoffs. That is, for $i=1,2$ and $j \equiv 3-i, h_{i}\left(D_{j}\right)=D_{j}, \tilde{u}_{i}(\tilde{X})=\tilde{X}^{\prime} \beta_{i}$ and $\delta_{i}(\tilde{X})=\delta_{i}$, where $\beta_{i}, \delta_{i}$ are constant parameters to be inferred. The specification is similar to Aradillas-Lopez (2010), except the independence between $\left(\epsilon_{i}\right)_{i \in N}$ and $X$ therein is replaced here by the weaker assumption of conditional independence between $\left(\epsilon_{1}, \epsilon_{2}\right)$ and $\left(X_{1}, X_{2}\right)$ given $\tilde{X}$.

We first show how to relate $\alpha_{i}, \delta_{i}, \beta_{i}$ to observable distributions in the semiparametric model. Given identification has been established for the general non-parametric case, our focus here is to propose convenient algebraic links between parameters and observable distributions under additional parametric restrictions. Consider any $\omega$ such that $0<p_{i}^{*}(X), p_{j}^{*}(X)<1$ and all coordinates in $\mathcal{W}_{2}(x), \mathcal{V}_{2}(x)$ and $\mathcal{V}_{2}(x) . \mathcal{W}_{1}(x)-\mathcal{V}_{1}(x) . \mathcal{W}_{2}(x)$ are non-zero almost everywhere on $\omega$. For $i=1,2$, $\alpha_{i}$ are identified as

$$
\alpha_{i}=\operatorname{sign}\left\{E\left[\left(p_{i, i}^{*}(X)-\frac{p_{i, j}^{*}(X) p_{j, i}^{*}(X)}{p_{j, j}^{*}(X)}\right) 1\{X \in \omega\}\right]\right\}
$$

and $\delta_{i}$ are identified as in equation (9) respectively. To attain identification of $\beta_{i}$ in this semi- 
parametric model based on Corollary 2, define for any $i$ and $x$

$$
Y_{i, H} \equiv \frac{\left[d_{i}-H\left(V_{i}(x)\right)\right]\left[1+\alpha_{i} \delta_{i} p_{j, i}^{*}(x)\right]}{f_{X_{i}}\left(x_{i} \mid x_{-i}\right)},
$$

where $V_{i}(x) \equiv \alpha_{i} x_{i}+\delta_{i} p_{j}^{*}(x)$, and $H$ is an arbitrary continuously differentiable distribution with a support contained in the support of $V_{i}(X)$ given $x_{-i}$ and an expectation equal to $\mu_{H}$. We will choose $H$ explicitly while estimating $\beta_{i}$ in Section 6.2 below. We maintain the following assumption throughout this section.

A5" (i) Given any $i$ and $\tilde{x}$, the support $\Omega_{V_{i} \mid x_{-i}}$ includes the support $\Omega_{\mathcal{S}_{i} \mid \tilde{x}}$ for all $x_{-i} \in \Omega_{X_{-i} \mid \tilde{x}}$; (ii) Given any $i$ and $\tilde{x}$, the density of $X_{i}$ given $x_{-i}$ is positive almost surely for all $x_{-i} \in \Omega_{X_{-i} \mid \tilde{x}}$. (iii) The sign of $\partial V_{i}(X) /\left.\partial X_{i}\right|_{X=x}$ equals the sign $\alpha_{i}$ for all $x \in \Omega_{X}$.

Condition A5" is more restrictive than A5' (stated in Section 5), in that A5" requires the set of $x_{-i}$ satisfying the three conditions in A5' to be the whole support of $X_{-i}$ given $\tilde{x}$. Intuitively, A5" can be satisfied when (i) conditional on any $x_{-i}$, the support of $X_{i}$ is sufficiently large relative to that of $\mathcal{S}_{i}$ given $\tilde{x}$; and $(i i) \delta_{i}(\tilde{x})$ and the density of $\epsilon_{i}$ given $\tilde{x}$ are both bounded above by constants that are relative small (in the sense stated in Appendix B). The condition that $f_{\epsilon_{i} \mid \tilde{x}}$ is bounded above by small constant is plausible especially in cases where the support of $\epsilon$ and $X_{e}$ are both large given $\tilde{x}$. We formalize these restrictions on model primitives which imply A5" in Appendix B.

Corollary 3 (Theorem 3) Suppose A1-4 and A5" hold and $\tilde{u}_{i}(\tilde{x})=\tilde{x}^{\prime} \beta_{i}, \delta_{i}(\tilde{x})=\delta_{i}$ for $i=1,2$. Suppose $\alpha_{i}, \delta_{i}$ are identified for both $i$. If $E\left(\tilde{X} \tilde{X}^{\prime}\right)$ has full rank, then

$$
\beta_{i}=\left[E\left(\tilde{X} \tilde{X}^{\prime}\right)\right]^{-1} E\left[\tilde{X}\left(Y_{i, H}-\mu_{H}\right)\right] .
$$

To prove Corollary 3 , apply the proof of Corollary 2 with $\tilde{u}_{i}(\tilde{X})=\tilde{X}^{\prime} \beta_{i}$ to get $\tilde{x}^{\prime} \beta_{i}=E\left[Y_{i, H} \mid\right.$

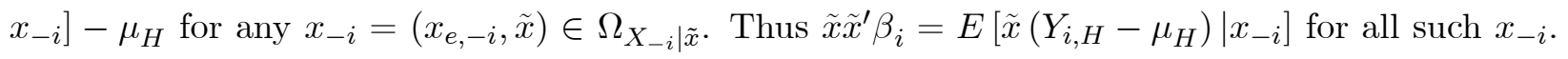
Integrating out $X_{-i}$ on both sides proves (17).

Sections 6.1 and 6.2 below define semiparametric estimators for $\alpha_{i}, \beta_{i}, \delta_{i}$ and derive their asymptotic distributions. Recovering these parameters requires conditioning on a set of states $\omega$ in (16) and (9). Because marginal effects of excluded regressors $X_{i}$ on the equilibrium choice probabilities $\left(p_{i}^{*}, p_{j}^{*}\right)$ are identified from data, the set can be estimated using sample analogs of the choice probabilities and the generated special regressors. For instance, $\omega$ can be estimated by $\hat{\omega}=\left\{x: \hat{p}_{i}(x)\right.$, $\hat{p}_{j}(x) \in(c, 1-c) ;\left|\hat{p}_{i, i}(x)\right|,\left|\hat{p}_{j, j}(x)\right|>c$ and $\left.\left|\hat{p}_{i, i}(x) \hat{p}_{j, j}(x)-\hat{p}_{i, j}(x) \hat{p}_{j, i}(x)\right|>c\right\}$, where $\hat{p}_{i}, \hat{p}_{j}, \hat{p}_{i, i}, \hat{p}_{j, j}$ are kernel estimators to be defined in Section 6.1 and $c$ is some constant close to 0. As $c$ is strictly above $0, \operatorname{Pr}\{\hat{\omega} \subset \omega\} \rightarrow 1$ by construction. Thus estimation errors in $\hat{\omega}$ has no bearing on the 
limiting distribution of estimators for the other parameters. In what follows, we do not account for estimation error in $\omega$ while establishing limiting distributions of our estimators for $\alpha_{i}, \delta_{i}$ and $\beta_{i}$. Such a task is left for future research. This choice allows us to better focus on dealing with estimation errors in $\hat{p}_{i}, \hat{p}_{j}, \hat{p}_{i, i}, \hat{p}_{i, j}$ as well as in $\hat{\delta}_{i}$ in our multi-step estimators below.

\subsection{Estimation of $\alpha_{i}$ and $\delta_{i}$}

We consider the case where $X$ consists of $J_{c}$ continuous and $J_{d}$ discrete coordinates (denoted by $X_{c} \equiv\left(X_{e}, \tilde{X}_{c}\right)$ and $\tilde{X}_{d}$ respectively). Econometricians observe $G$ independent games in data, with each indexed by $g$. We show $\alpha_{i}$ can be estimated at an arbitrarily fast rate and $\delta_{i}$ at the root-N rate. Let $\gamma$ denote a vector of functions $\left(\gamma_{0}, \gamma_{1}, \gamma_{2}\right)$, where $\gamma_{0}(x) \equiv f\left(x_{c} \mid \tilde{x}_{d}\right) f_{d}\left(\tilde{x}_{d}\right)$ and $\gamma_{k}(x) \equiv E\left[D_{k} \mid x\right] f\left(x_{c} \mid \tilde{x}_{d}\right) f_{d}\left(\tilde{x}_{d}\right)$ for $k=1,2$, with $f(. \mid),. E(. \mid$.$) being conditional densities and$ expectations, and $f_{d}($.$) being a probability mass function for \tilde{X}_{d}$ respectively. Define $\gamma_{k, i}(x) \equiv \frac{\partial \gamma_{k}(x)}{\partial X_{i}}$ for $k \in\{0,1,2\}$ and $i \in\{1,2\}$. Define a 9-by-1 vector:

$$
\gamma_{(1)} \equiv\left(\gamma_{0}, \gamma_{1}, \gamma_{2}, \gamma_{0,1}, \gamma_{0,2}, \gamma_{1,1}, \gamma_{1,2}, \gamma_{2,1}, \gamma_{2,2}\right)
$$

Let $\gamma_{(1)}^{*}$ and $\hat{\gamma}_{(1)}$ denote true parameters in the data-generating process and their corresponding kernel estimates respectively. (For notation simplicity, we sometimes suppress their arguments $X$ when there is no ambiguity.) We use the sup-norm $\sup _{x \in \Omega_{X}}\|$.$\| for spaces of functions with domain$ $\Omega_{X}$.

For $i=1,2$, let $j \equiv 3-i$ and define

$$
A^{i} \equiv E\left[m_{A}^{i}\left(X ; \gamma_{(1)}^{*}\right) 1\{X \in \omega\}\right] \text { and } \Delta^{i} \equiv E\left[m_{\Delta}^{i}\left(X ; \gamma_{(1)}^{*}\right) \mid X \in \omega\right]
$$

where

$$
\begin{aligned}
& m_{A}^{i}\left(x ; \gamma_{(1)}\right) \equiv \frac{\gamma_{i, i}(x) \gamma_{0}(x)-\gamma_{i}(x) \gamma_{0, i}(x)}{\gamma_{0}(x) \gamma_{0}(x)}-\frac{\gamma_{i, j}(x) \gamma_{0}(x)-\gamma_{i}(x) \gamma_{0, j}(x)}{\gamma_{0}(x) \gamma_{0}(x)} \frac{\gamma_{j, i}(x) \gamma_{0}(x)-\gamma_{j}(x) \gamma_{0, i}(x)}{\gamma_{j, j}(x) \gamma_{0}(x)-\gamma_{j}(x) \gamma_{0, j}(x)} \\
& m_{\Delta}^{i}\left(x ; \gamma_{(1)}\right) \equiv\left(\frac{\gamma_{i, i}(x) \gamma_{0}(x)-\gamma_{i}(x) \gamma_{0, i}(x)}{\gamma_{i, j}(x) \gamma_{0}(x)-\gamma_{i}(x) \gamma_{0, j}(x)}-\frac{\gamma_{j, i}(x) \gamma_{0}(x)-\gamma_{j}(x) \gamma_{0, i}(x)}{\gamma_{j, j}(x) \gamma_{0}(x)-\gamma_{j}(x) \gamma_{0, j}(x)}\right)^{-1}\left(\frac{\gamma_{j, j} \gamma_{0}-\gamma_{j} \gamma_{0, j}}{\gamma_{0}(x) \gamma_{0}(x)}\right)^{-1}
\end{aligned}
$$

Estimate $A^{i}, \Delta^{i}$ by their sample analogs as

$$
\begin{aligned}
& \hat{A}^{i} \equiv \frac{1}{G} \sum_{g} w_{g} m_{A}^{i}\left(x_{g} ; \hat{\gamma}_{(1)}\right) ; \\
& \hat{\Delta}^{i} \equiv\left(\sum_{g} w_{g}\right)^{-1} \sum_{g} w_{g} m_{\Delta}^{i}\left(x_{g} ; \hat{\gamma}_{(1)}\right),
\end{aligned}
$$

where $w_{g}$ is a short-hand for $1\left\{x_{g} \in \omega\right\}$. Let $K($.$) be a product kernel for continuous coordinates$ in $X_{c}$, and $\sigma$ be a sequence of bandwidths with $\sigma \rightarrow 0$ as $G \rightarrow+\infty$. To simplify exposition, define $d_{g, 0} \equiv 1$ for all $g \leq G$. Kernel estimates for $\gamma_{k}^{*}$ and its partial derivatives with respect to excluded regressors are

$$
\begin{aligned}
& \hat{\gamma}_{k}\left(x_{c}, \tilde{x}_{d}\right) \equiv \frac{1}{G} \sum_{g} d_{g, k} K_{\sigma}\left(x_{g, c}-x_{c}\right) 1\left\{\tilde{x}_{g, d}=\tilde{x}_{d}\right\} \\
& \hat{\gamma}_{k, i}\left(x_{c}, \tilde{x}_{d}\right) \equiv \frac{1}{G} \sum_{g} d_{g, k} K_{\sigma, i}\left(x_{g, c}-x_{c}\right) 1\left\{\tilde{x}_{g, d}=\tilde{x}_{d}\right\}
\end{aligned}
$$


for $k=0,1,2$ and $i=1,2$, where $K_{\sigma}(.) \equiv \sigma^{-J_{c}} K(. / \sigma), K_{\sigma, i}(.) \equiv \sigma^{-J_{c}} \partial K(. / \sigma) / \partial X_{i}{ }^{9} \quad$ Our estimators for $\alpha_{i}, \delta_{i}$ are

$$
\hat{\alpha}_{i}=\operatorname{sign}\left(\hat{A}^{i}\right) ; \hat{\delta}_{i}=\hat{\alpha}_{i} \hat{\Delta}^{i} .
$$

Let $F_{Z}^{*}, f_{Z}^{*}$ denote the true distribution and the density of $Z \equiv\left(X, D_{1}, D_{2}\right)$ in the data-generating process.

Assumption $\mathbf{S}$ (i) $\boldsymbol{\gamma}^{*}$ is continuously differentiable in $x_{c}$ to an order of $\bar{m} \geq 2$ given any $\tilde{x}_{d}$, and the derivatives are continuous and uniformly bounded over an open set containing $\omega$. (ii) $\boldsymbol{\gamma}_{(1)}^{*}$ is bounded away from 0 over $\omega$. (iii) Let $V A R_{u_{i}}(x)$ be the variance of $u_{i}=d_{i}-p_{i}^{*}(x)$ given $x$. There exists $\xi>0$ such that $\left[V A R_{u_{i}}(x)\right]^{1+\xi} f^{*}\left(x_{c} \mid \tilde{x}_{d}\right)$ is uniformly bounded over $\omega$. For any $\tilde{x}_{d}$, both $p_{i}^{*}(x)^{2} f^{*}\left(x_{c} \mid \tilde{x}_{d}\right)$ and $V A R_{u_{i}}(x) f^{*}\left(x_{c} \mid \tilde{x}_{d}\right)$ are continuous in $x_{c}$ and uniformly bounded over $\omega$.

Assumption K The kernel $K($.$) satisfies: (i) K($.$) is bounded and differentiable in X_{e}$ of order $\bar{m}$ and the partial derivatives are bounded over $\omega$; (ii) $\int|K(u)| d u<\infty, \int K(u) d u=1, K($.) has zero moments up to the order of $m$ (where $m \leq \bar{m}$ ), and $\int\|u\|^{m}|K(u)| d u<\infty$; and (iii) $K($.$) is$ zero outside a bounded set.

Assumption B $\sqrt{G} \sigma^{m} \rightarrow 0$ and $\left(\frac{\sqrt{G}}{\ln G}\right) \sigma^{\left(J_{c}+2\right)} \rightarrow+\infty$ as $G \rightarrow+\infty$.

A sequence of bandwidths $\sigma$ that satisfies Assumption B exists, as long as the parameter for smoothness $m$ is sufficiently large relative to $J_{c}$. Under Assumptions S, K and B, $\sup _{x \in \omega} \| \hat{\gamma}_{(1)}(x)-$ $\gamma_{(1)}^{*}(x) \|=o_{p}\left(G^{-1 / 4}\right)$. This ensures the remainder term from linearization of $\frac{1}{G} \sum_{g} w_{g} m_{A}^{i}\left(x_{g} ; \hat{\gamma}_{(1)}\right)$ at $\gamma_{(1)}^{*}$ diminishes at a rate faster than $\sqrt{G}$.

Assumption $\mathbf{W}$ The set $\omega$ is convex and contained in an open subset of $\Omega_{X}$.

The convexity of $\omega$ implies that for all $i$ the the set $\left\{x_{i}:\left(x_{i}, x_{-i}\right) \in \omega\right\}$ must be an interval. Denote end-points of the interval by $l_{i}\left(x_{-i}\right)$ and $h_{i}\left(x_{-i}\right)$ for $i$ and $x_{-i}$. This property implies for any function $\mathcal{G}(x)$, the expression $\int 1\{x \in \omega\} \mathcal{G}(x) d F_{X}^{*}$ can be written as $\iint_{l_{i}\left(x_{-i}\right)}^{h_{i}\left(x_{-i}\right)} \mathcal{G}(x) \gamma_{0}^{*}(x) d x_{i} d x_{-i}$, which comes in handy as we derive the correction terms in the limiting distribution of $\sqrt{G}\left(\hat{A}^{i}-A^{i}\right)$ and $\sqrt{G}\left(\hat{\Delta}^{i}-\Delta^{i}\right)$ due to the use of kernel estimates $\hat{\gamma}_{(1)}$. That $\omega$ is contained in an open subset of $\Omega_{X}$ is necessary for derivatives of $\gamma_{(1)}, \gamma_{(1)}^{*}$ with respect to $x$ to be well-defined over $\omega$.

To specify correction terms in the limiting distribution, we need to introduce additional notations. Let $w_{x} \equiv 1\{x \in \omega\}$ for the convex set $\omega$ satisfying NDS. For $i=1,2$, let $\tilde{D}_{i, A}(x)$ (and $\left.\tilde{D}_{i, \Delta}(x)\right)$ denote two 9-by-1 vectors that consist of derivatives of $m_{A}^{i}\left(x ; \gamma_{(1)}\right)$ (and $m_{\Delta}^{i}\left(x ; \gamma_{(1)}\right)$ respectively) with respect to the ordered vector $\left(\gamma_{0}, \gamma_{i}, \gamma_{j}, \gamma_{0, i}, \gamma_{0, j}, \gamma_{i, i}, \gamma_{i, j}, \gamma_{j, i}, \gamma_{j, j}\right)$ at $\gamma_{(1)}^{*}$.

\footnotetext{
${ }^{9}$ Alternatively, we can also replace the indicator functions in $\hat{\gamma}_{k}$ and $\hat{\gamma}_{k, i}$ with smoothing product kernels for the discrete covariations as well. Denote such a joint product kernel for all coordinates in $X$ as $\tilde{K}$. Bierens (1985) showed uniform convergence of $\hat{\gamma}_{k}$ in probability of can be established as long as $\sqrt{G} \int \sup _{\left|u_{d}\right|>\zeta / \sigma}\left|\tilde{K}\left(u_{c}, u_{d}\right)\right| d u_{c} \rightarrow 0$ for all $\zeta>0$.
} 
Let $\tilde{D}_{i, A, k}$ (and $\tilde{D}_{i, \Delta, k}$ ) denote the $k$-th coordinate in $\tilde{D}_{i, A}$ (and $\tilde{D}_{i, \Delta}$ ) for all $k$. For $s=i, j$, let $\tilde{D}_{i, A, k}^{(s)}(x)$ (and $\tilde{D}_{i, \Delta, k}^{(s)}(x)$ ) denote the derivative of $\tilde{D}_{i, A, k}(X) \gamma_{0}^{*}(X)$ (and $\tilde{D}_{i, \Delta, k}(X) \gamma_{0}^{*}(X)$ ) with respect to the excluded regressor $X_{s}$ at $x$. We include the detailed forms of $\tilde{D}_{i, A}, \tilde{D}_{i, \Delta}, \tilde{D}_{i, A}^{(s)}, \tilde{D}_{i, \Delta}^{(s)}$ in the supplement of this article (Lewbel and Tang (2012)).

Let $\psi_{A}^{i} \equiv\left(\psi_{A, 0}^{i}, \psi_{A, i}^{i}, \psi_{A, j}^{i}\right)$, where

$$
\begin{aligned}
\psi_{A, 0}^{i}\left(x ; \gamma_{(1)}^{*}\right) & \equiv w_{x}\left[\tilde{D}_{i, A, 1}(x) \gamma_{0}^{*}(x)-\tilde{D}_{i, A, 4}^{(i)}(x)-\tilde{D}_{i, A, 5}^{(j)}(x)\right]+\mathcal{I}_{A, 1}^{i}(x) \\
\psi_{A, i}^{i}\left(x ; \gamma_{(1)}^{*}\right) & \equiv w_{x}\left[\tilde{D}_{i, A, 2}(x) \gamma_{0}^{*}(x)-\tilde{D}_{i, A, 6}^{(i)}(x)-\tilde{D}_{i, A, 7}^{(j)}(x)\right]+\mathcal{I}_{A, 2}^{i}(x) ; \\
\psi_{A, j}^{i}\left(x ; \gamma_{(1)}^{*}\right) & \equiv w_{x}\left[\tilde{D}_{i, A, 3}(x) \gamma_{0}^{*}(x)-\tilde{D}_{i, A, 8}^{(i)}(x)-\tilde{D}_{i, A, 9}^{(j)}(x)\right]+\mathcal{I}_{A, 3}^{i}(x),
\end{aligned}
$$

with

$$
\mathcal{I}_{A, 1}^{i}(x) \equiv\left(\begin{array}{c}
1\left\{x_{i}=h_{i}\left(x_{-i}\right)\right\} \tilde{D}_{i, A, 4}(x) \gamma_{0}^{*}(x)-1\left\{x_{i}=l_{i}\left(x_{-i}\right)\right\} \tilde{D}_{i, A, 4}(x) \gamma_{0}^{*}(x) \\
+1\left\{x_{j}=h_{j}\left(x_{-j}\right)\right\} \tilde{D}_{i, A, 5}(x) \gamma_{0}^{*}(x)-1\left\{x_{j}=l_{j}\left(x_{-j}\right)\right\} \tilde{D}_{i, A, 5}(x) \gamma_{0}^{*}(x)
\end{array}\right) .
$$

The term $\mathcal{I}_{A, 2}^{i}(x)$ is defined by replacing $\tilde{D}_{i, A, 4}, \tilde{D}_{i, A, 5}$ in (20) with $\tilde{D}_{i, A, 6}, \tilde{D}_{i, A, 7}$ respectively. Likewise, $\mathcal{I}_{A, 3}^{i}(x)$ is defined by replacing $\tilde{D}_{i, A, 4}, \tilde{D}_{i, A, 5}$ in (20) with $\tilde{D}_{i, A, 8}, \tilde{D}_{i, A, 9}$ respectively. Similarly, define $\psi_{\Delta}^{i} \equiv\left(\psi_{\Delta, 0}^{i}, \psi_{\Delta, i}^{i}, \psi_{\Delta, j}^{i}\right)$ by replacing $\tilde{D}_{i, A}, \tilde{D}_{i, A}^{(s)}, \mathcal{I}_{A}^{i}$ in $\psi_{A, s}^{i}$ with $\tilde{D}_{i, \Delta,}, \tilde{D}_{i, \Delta}^{(s)}, \mathcal{I}_{\Delta}^{i}$ respectively, where $\mathcal{I}_{\Delta}^{i} \equiv\left(\mathcal{I}_{\Delta, 1}^{i}, \mathcal{I}_{\Delta, 2}^{i}, \mathcal{I}_{\Delta, 3}^{i}\right)$ is defined by replacing $\tilde{D}_{i, A, k}$ in the definition of $\mathcal{I}_{A}^{i}$ with $\tilde{D}_{i, \Delta, k}$ for all $k$.

Assumption $\mathbf{D}(i)$ For both $i$ and $s=1,2, \tilde{D}_{i, A}, \tilde{D}_{i, \Delta}, \tilde{D}_{i, A}^{(s)}, \tilde{D}_{i, \Delta}^{(s)}$ are continuous and bounded over an open set containing $\omega$. (ii) The second order derivatives of $m_{A}^{i}\left(x ; \gamma_{(1)}\right)$ and $m_{\Delta}^{i}\left(x ; \gamma_{(1)}\right)$ with respect to $\gamma_{(1)}$ are bounded at $\gamma_{(1)}^{*}$ over an open set containing $\omega$. (iii) For both $i$, there exists constants $c_{A}^{i}, c_{\Delta}^{i}>0$ such that $E\left[\sup _{\|\eta\| \leq c_{A}^{i}}\left\|\psi_{A, s}^{i}(x+\eta)\right\|^{4}\right]<\infty$ and $E\left[\sup _{\|\eta\| \leq c_{\Delta}^{i}}\left\|\psi_{\Delta, s}^{i}(x+\eta)\right\|^{4}\right]$ $<\infty$ for $s=0,1,2$.

As before, we let $D \equiv\left[D_{0}, D_{1}, D_{2}\right]^{\prime}$ (with $D_{0} \equiv 1$ ) in order to simplify notations, and use lower case $d$ and $d_{1}, d_{2}$ for corresponding realized values. Conditions D-(i),(ii) ensure the remainder term after linearizing the sample moments around the true functions $\gamma_{(1)}^{*}$ in DGP vanishes at a rate faster than $\sqrt{G}$. Applying the V-statistic projection theorem (Lemma 8.4 in Newey and McFadden (1994)), we also use these conditions to show that the difference between $\frac{1}{G} \sum_{g} w_{g} m_{A}^{i}\left(x_{g} ; \hat{\gamma}_{(1)}\right)$ and $\frac{1}{G} \sum_{g} w_{g} m_{A}^{i}\left(x_{g} ; \gamma_{(1)}^{*}\right)$ can be written as $\int w_{x}\left(\hat{\gamma}_{(1)}-\gamma_{(1)}^{*}\right)^{\prime} \tilde{D}_{i, A} d F_{X}^{*}$ plus a term that is $o_{p}\left(G^{-1 / 2}\right)$, where $F_{X}^{*}$ is the true marginal distribution of $X$ in the DGP. Furthermore, by definition and the smoothness properties in $\mathrm{S}$, for any $\gamma$ that is twice continuously differentiable in excluded regressors $X_{e}, \psi_{A}^{i} \equiv\left(\psi_{A, 0}^{i}, \psi_{A, 1}^{i}, \psi_{A, 2}^{i}\right)$ satisfies:

$$
\int w_{x}\left(\gamma_{(1)}\right)^{\prime} \tilde{D}_{i, A} d F_{X}^{*}=\int \sum_{s=0,1,2} \psi_{A, s}^{i}(x) \gamma_{s}(x) d x
$$

Using (21), the difference $\int w_{x}\left(\hat{\gamma}_{(1)}-\gamma_{(1)}^{*}\right)^{\prime} \tilde{D}_{i, A} d F_{X}^{*}$ can be written as $\int \tilde{\nu}_{A}(z) d \tilde{F}_{Z}$, where $\tilde{\nu}_{A}(z) \equiv \psi_{A}^{i}(x) d-E\left[\psi_{A}^{i}(X) D\right]$ and $\tilde{F}_{Z}$ is some smoothed version of the empirical distribution 
of $Z \equiv\left(X, D_{i}, D_{j}\right)$. Condition (iii) in Assumption D is used for showing the difference between $\int \tilde{\nu}_{A}(z) d \tilde{F}_{Z}$ and $\frac{1}{G} \sum_{g \leq G} \tilde{\nu}_{A}\left(z_{g}\right)$ is $o_{p}\left(G^{-1 / 2}\right)$. Thus the difference between $\frac{1}{G} \sum_{g} w_{g} m_{A}^{i}\left(x_{g} ; \hat{\gamma}_{(1)}\right)$ and $\frac{1}{G} \sum_{g}\left[w_{g} m_{A}^{i}\left(x_{g} ; \gamma_{(1)}^{*}\right)+\tilde{\nu}_{A}\left(z_{g}\right)\right]$ is $o_{p}\left(G^{-1 / 2}\right)$. Under the same conditions and steps for derivations, a similar result holds with $m_{A}^{i}, \tilde{D}_{i, A}$ and $\psi_{A, s}^{i}$ replaced by $m_{\Delta}^{i}, \tilde{D}_{i, \Delta}$ and $\psi_{\Delta, s}^{i}$ respectively. For condition (iii) in Assumption $\mathrm{D}$ to hold, it is sufficient that for $i=1,2$, there exists constants $c_{A}^{i}, c_{\Delta}^{i}>0$ with $E\left[\sup _{\|\eta\| \leq c_{A}^{i}}\left\|\tilde{D}_{i, A, s}(x+\eta)\right\|^{4}\right]<\infty$ and $E\left[\sup _{\|\eta\| \leq c_{\Delta}^{i}}\left\|\tilde{D}_{i, \Delta, s}(x+\eta)\right\|^{4}\right]<\infty$ for $s=0,1,2$, and $E\left[\sup _{\|\eta\| \leq c_{A}^{i}}\left\|\tilde{D}_{i, A, k}^{(s)}(x+\eta)\right\|^{4}\right]<\infty$ and $E\left[\sup _{\|\eta\| \leq c_{\Delta}^{i}}\left\|\tilde{D}_{i, \Delta, k}^{(s)}(x+\eta)\right\|^{4}\right]<\infty$ for $s=1,2$ and $k \in\{4,5,6,7\}$.

Assumption $\mathbf{R}(i)$ There exists an open neighborhood $\mathcal{N}_{\gamma}$ around $\gamma_{(1)}^{*}$ with $E\left[\sup _{\gamma_{(1)} \in \mathcal{N}_{\gamma}} \| w_{X} m_{\Delta}^{i}(X\right.$; $\left.\left.\gamma_{(1)}\right) \|\right]<\infty$. (ii) $E\left[\left\|w_{X} m_{A}^{i}\left(X ; \gamma_{(1)}^{*}\right)+\tilde{\nu}_{A}(Z)\right\|^{2}\right]<\infty$ and $E\left[\left\|w_{X}\left[m_{\Delta}^{i}\left(X ; \gamma_{(1)}^{*}\right)-\Delta^{i}\right]+\tilde{\nu}_{\Delta}(Z)\right\|^{2}\right]$ $<\infty$.

Condition (i) in Assumption R ensures $\frac{1}{G} \sum_{g \leq G} w_{g} m_{\Delta}^{i}\left(X ; \hat{\gamma}_{(1)}\right) \stackrel{p}{\longrightarrow} E\left[w_{X} m_{\Delta}^{i}\left(X ; \boldsymbol{\gamma}_{(1)}^{*}\right)\right]$ when $\hat{\gamma}_{(1)} \stackrel{p}{\longrightarrow} \gamma_{(1)}^{*}$. This is useful for deriving the correction term that results from the use of sample proportions $\frac{1}{G} \sum_{g} w_{g}$ instead of the population probability $\rho_{0} \equiv E\left(w_{X}\right) \equiv \operatorname{Pr}(X \in \omega)$ while estimating $\Delta^{i}$. Condition (ii) in Assumption $\mathrm{R}$ is necessary for the Central Limit Theorem to apply.

Lemma 1 Suppose $S, K, B, D, W$ and $R$ hold. Then for $i=1,2$,

$$
\begin{aligned}
& \sqrt{G}\left(\hat{A}^{i}-A^{i}\right) \stackrel{d}{\rightarrow} \mathcal{N}\left(0, \operatorname{Var}\left[w_{X} m_{A}^{i}\left(X ; \gamma_{(1)}^{*}\right)+\tilde{\nu}_{A}^{i}(Z)\right]\right) \\
& \sqrt{G}\left(\hat{\Delta}^{i}-\Delta^{i}\right) \stackrel{d}{\longrightarrow} \mathcal{N}\left(0, \rho_{0}^{-2} \operatorname{Var}\left[w_{X}\left(m_{\Delta}^{i}\left(X ; \gamma_{(1)}^{*}\right)-\Delta^{i}\right)+\tilde{\nu}_{\Delta}^{i}(Z)\right]\right)
\end{aligned}
$$

where $\tilde{\nu}_{A}^{i}(z) \equiv \psi_{A}^{i}(x) d-E\left[\psi_{A}^{i}(X) D\right]$ and $\tilde{\nu}_{\Delta}^{i}(z) \equiv \psi_{\Delta}^{i}(x) d-E\left[\psi_{\Delta}^{i}(X) D\right]$.

Lemma 1 follows from the steps in Section 8 of Newey and McFadden (1994) delineated above. The proof of this lemma is included in the supplement of this paper (Lewbel and Tang (2012)). It follows from Lemma 1 and Slutsky's Theorem that $\hat{\delta}_{i}$ converges to $\delta_{i}$ at the parametric rate.

Theorem 4 Suppose A1-4 and Assumptions $S, K, B, W, D$ and $R$ hold. Then for $i=1,2$, $\operatorname{Pr}\left(\hat{\alpha}_{i}=\alpha_{i}\right) \rightarrow 1$ and $\sqrt{G}\left(\hat{\delta}_{i}-\delta_{i}\right) \stackrel{d}{\rightarrow} \mathcal{N}\left(0, \Sigma_{\Delta}^{i}\right)$, where $\Sigma_{\Delta}^{i}$ is the limiting variance of $\sqrt{G}\left(\hat{\Delta}^{i}-\Delta^{i}\right)$ in (22).

Proof of Theorem 4 is included in the supplement of this paper. The rate of convergence for each $\hat{\alpha}_{i}$ is arbitrarily fast because each is defined as the sign of an estimator that converges at the root- $\mathrm{N}$ rate to its population counterpart. The parametric rate can be attained by $\hat{\delta}_{i}$ because it takes the form of a sample moment involving sufficiently regular preliminary nonparametric estimates. Both properties come in handy as we derive the limiting behavior of our estimator for $\beta_{i}$. 


\subsection{Estimation of $\beta_{i}$}

Since $\operatorname{Pr}\left(\hat{\alpha}_{i}=\alpha\right) \rightarrow 1$, we derive asymptotic properties of the estimator for $\beta_{i}$ treating $\alpha_{i}$ as if it is known. In some applications $\alpha_{i}$ 's are in fact known a priori, as in the entry game example where $X_{i}$ (observed components in fixed costs) must negatively affect profits. Without loss of generality, let $\alpha_{i}=-1$ in what follows as would be the case in the entry game.

Let $v_{i}^{l}<v_{i}^{h}$ be any two values that known to be in the support of $V_{i}(X)$ given $x_{-i}=\left(x_{e,-i}, \tilde{x}\right)$. The dependence of $v_{i}^{l}, v_{i}^{h}$ (and the choice of the smooth distribution $H$ ) on $x_{-i}$ is suppressed to simplify the notation throughout this section. Choices of $v_{i}^{l}, v_{i}^{h}$ are feasible in estimation as the support $\Omega_{V_{i} \mid x_{-i}}$ can be estimated by inf and sup of $-t+\hat{\delta}_{i} \hat{p}_{j, i}\left(t, x_{-i}\right)$ for $t \in \Omega_{X_{i} \mid x_{-i}}$. We take $v_{i}^{l}, v_{i}^{h}$ as known while deriving the asymptotic distribution of $\hat{\beta}_{i}$. Define a smooth distribution by

$$
H(v) \equiv \mathcal{K}\left(2\left(\frac{v-v_{i}^{l}}{v_{i}^{h}-v_{i}^{l}}\right)-1\right) \text { for all } v \in\left[v_{i}^{l}, v_{i}^{h}\right],
$$

where $\mathcal{K}$ denotes the integrated bi-weight kernel function. That is, $\mathcal{K}(u)=0$ if $u<-1 ; \mathcal{K}(u)=1$ if $u>1$; and $\mathcal{K}(u)=\frac{1}{16}\left(8+15 u-10 u^{3}+3 u^{5}\right)$ for $-1 \leq u \leq 1$. The integrated bi-weight kernel is continuously differentiable with its first-order derivative $\mathcal{K}^{\prime}(u)$ equal to 0 if $|u|>1$ and equal to the bi-weight kernel $\frac{15}{16}\left(1-u^{2}\right)^{2}$ otherwise. This distribution $H$ depends on $x_{-i}$ through its support $\left[v_{i}^{l}, v_{i}^{h}\right]$, and it is symmetric around its expectation $\mu_{H} \equiv \frac{1}{2}\left(v_{i}^{h}+v_{i}^{l}\right)$. This choice of $H$ is not necessary, but is convenient for our estimator and satisfies all the required conditions.

For $i=1,2$ and $j \equiv 3-i$, define:

$$
\begin{aligned}
\hat{p}_{j}(x) & \equiv \hat{\gamma}_{j}(x) / \hat{\gamma}_{0}(x) ; \quad \hat{p}_{j, i}(x) \equiv\left[\hat{\gamma}_{0}(x)\right]^{-2}\left[\hat{\gamma}_{j, i}(x) \hat{\gamma}_{0}(x)-\hat{\gamma}_{j}(x) \hat{\gamma}_{0, i}(x)\right] \\
\widehat{\mathcal{H}}_{i}(x) & \equiv H\left(-x_{i}+\hat{\delta}_{i} \hat{p}_{j}(x)\right) ; \text { and } \widehat{\mathcal{V}}_{(i)}(x) \equiv 1-\hat{\delta}_{i} \hat{p}_{j, i}(x) .
\end{aligned}
$$

We use $\widehat{\mathcal{H}}_{i}(x)$ as an estimator for $H\left(V_{i}(x)\right)$ and $\widehat{\mathcal{V}}_{(i)}(x)$ an estimator for $-\partial V_{i}(x) / \partial X_{i}$ in the case with $\alpha_{i}=-1$.

For $i=1,2$ and $j=3-i$, let $p_{j} \equiv \gamma_{j} / \gamma_{0}$ and $p_{j, i} \equiv\left(\gamma_{j, i} \gamma_{0}-\gamma_{j} \gamma_{0, i}\right) / \gamma_{0}^{2}$; and $\gamma_{(1, i)} \equiv$ $\left(\gamma_{0}, \gamma_{j}, \gamma_{0, i}, \gamma_{j, i}\right)$ denote a subvector of $\gamma_{(1)}$. For any $x$, the conditional density $f\left(x_{i} \mid x_{-i}\right)$ is a functional of $\gamma_{0}$ as $\gamma_{0}\left(x_{i}, x_{-i}\right) / \int \gamma_{0}\left(t, x_{-i}\right) d t$. Let $\gamma_{(1, i)}^{*}, p_{j}^{*}, p_{j, i}^{*}$ denote true functions in the datagenerating process, and $\hat{\gamma}_{(1, i)}, \hat{p}_{j}, \hat{p}_{j, i}$ to denote kernel estimates.For each game $g \leq G$ and both $i=1,2$, define

$$
\hat{y}_{g, i} \equiv \frac{\left[d_{g, i}-\widehat{\mathcal{H}}_{i}\left(x_{g}\right)\right] \widehat{\mathcal{V}}_{(i)}\left(x_{g}\right) \int \hat{\gamma}_{0}\left(t, x_{g,-i}\right) d t}{\hat{\gamma}_{0}\left(x_{g}\right)} .
$$

Our estimator for $\beta_{i}$ is given by $\hat{\beta}_{i} \equiv\left(\sum_{g} \tilde{x}_{g}^{\prime} \tilde{x}_{g}\right)^{-1}\left(\sum_{g} \tilde{x}_{g}^{\prime}\left(\hat{y}_{g, i}-\mu_{H}\right)\right)$.

Assumption S' (a) Assumption $S$ holds with the set $\omega$ therein replaced by $\Omega_{X}$. (b) the true density $f^{*}\left(x_{i} \mid x_{-i}\right)$ is bounded above and away from zero by some positive constant over $\Omega_{X}$. 
Along with kernel and bandwidth conditions in Assumptions $\mathrm{K}$ and B, conditions (a) and (b) in Assumption S' ensure $\sup _{x \in \Omega_{X}}\left\|\hat{\gamma}_{(1, i)}-\gamma_{(1, i)}^{*}\right\|$ converges in probability to 0 at rates faster than $G^{1 / 4}$. Bounding $\gamma^{*}(x)$ and $f^{*}\left(x_{i} \mid x_{-i}\right)$ away from zero over the support $\Omega_{X}$ and $\Omega_{X_{i} \mid x_{-i}}$ helps attain the stochastic boundedness of $\sqrt{G}\left(\hat{\beta}_{i}-\beta_{i}\right)$.

With the excluded regressors $X_{e}$ continuously distributed with positive densities almost everywhere, this requires the support of excluded regressors given $X_{-i}$ to be bounded. Thus in order for the support of $V_{i}$ given $x_{-i}$ to cover that of $-\tilde{u}_{i}(\tilde{x})+\epsilon_{i}$, it is necessary that the support of $\epsilon$ given $\tilde{X}$ is also bounded. (See the discussion in the next section regarding outcomes if boundedness is violated.) Condition (a) in Assumption S' also implies $p_{j, i}^{*}(x)$ is bounded above over $\Omega_{X}$ for $i=1,2$ and $j=3-i$.

Define $m_{B}^{i}\left(z ; \delta_{i}, \gamma_{(1, i)}\right)$ for $i=1,2$ as

$$
m_{B}^{i}\left(z ; \delta_{i}, \gamma_{(1, i)}\right) \equiv \tilde{x}^{\prime}\left\{\left[d_{i}-H\left(-x_{i}+\delta_{i} p_{j}(x)\right)\right] \frac{1-\delta_{i} p_{j, i}(x)}{f\left(x_{i} \mid x_{-i}\right)}-\mu_{H}\right\},
$$

with $z \equiv\left(d_{1}, d_{2}, x\right)$ and $p_{j}, p_{j, i}, f_{X_{i} \mid x_{-i}}$ being functions of $\gamma_{(1, i)}$ defined above. By definition,

$$
\hat{\beta}_{i}=\left(\sum_{g} \tilde{x}_{g}^{\prime} \tilde{x}_{g}\right)^{-1} \sum_{g} m_{B}^{i}\left(z_{g} ; \hat{\delta}_{i}, \hat{\gamma}_{(1, i)}\right) .
$$

For $i=1,2$ and $j=3-i$, define

$$
\begin{aligned}
& \left.\tilde{D}_{i, B, \delta}\left(z ; \delta_{i}, \gamma_{(1, i)}\right) \equiv \frac{\partial}{\partial \tilde{\delta}_{i}} m_{B}^{i}\left(z ; \tilde{\delta}_{i}, \gamma_{(1, i)}\right)\right|_{\tilde{\delta}_{i}=\delta_{i}} \\
= & \tilde{x}^{\prime}\left\{-H^{\prime}\left(-x_{i}+\delta_{i} p_{j}(x)\right) \frac{p_{j}(x)\left[1-\delta_{i} p_{j, i}(x)\right]}{f\left(x_{i} \mid x_{-i}\right)}-\left[d_{i}-H\left(-x_{i}+\delta_{i} p_{j}(x)\right)\right] \frac{p_{j, i}(x)}{f\left(x_{i} \mid x_{-i}\right)}\right\} .
\end{aligned}
$$

For $i=1,2$ and $j=3-i$, denote partial derivatives of $m_{B}^{i}\left(z ; \delta_{i}, \gamma_{(1, i)}\right)$ with respect to components of $\gamma_{(1, i)}(x)$ at $\delta_{i}$ and $\gamma_{(1, i)}^{*}(x)$ as:

$$
\begin{aligned}
\tilde{D}_{i, B, 1}(z) & \equiv \frac{\partial m_{B}^{i}\left(z ; \delta_{i}, \gamma_{(1, i)}^{*}\right)}{\partial p_{j}^{*}(x)} \frac{\partial p_{j}^{*}(x)}{\partial \gamma_{0}(x)}+\frac{\partial m_{B}^{i}\left(z ; \delta_{i}, \gamma_{(1, i)}^{*}\right)}{\partial p_{j, i}^{*}(x)} \frac{\partial p_{j, i}^{*}(x)}{\partial \gamma_{0}(x)} \\
\tilde{D}_{i, B, 2}(z) & \equiv \frac{\partial m_{B}^{i}\left(z ; \delta_{i}, \gamma_{(1, i)}^{*}\right)}{\partial p_{j}^{*}(x)} \frac{\partial p_{j}^{*}(x)}{\partial \gamma_{j}(x)}+\frac{\partial m_{B}^{i}\left(z ; \delta_{i}, \gamma_{(1, i)}^{*}\right)}{\partial p_{j, i}^{*}(x)} \frac{\partial p_{j, i}^{*}(x)}{\partial \gamma_{j}(x)} \\
\tilde{D}_{i, B, 3}(z) & \equiv \frac{\partial m_{B}^{i}\left(z ; \delta_{i}, \gamma_{(1, i)}^{*}\right)}{\partial p_{j, i}^{*}(x)} \frac{\partial p_{j, i}^{*}(x)}{\partial \gamma_{0, i}(x)}, \text { and } \tilde{D}_{i, B, 4}(z) \equiv \frac{\partial m_{B}^{i}\left(z ; \delta_{i}, \gamma_{(1, i)}^{*}\right)}{\partial p_{j, i}^{*}(x)} \frac{\partial p_{j, i}^{*}(x)}{\partial \gamma_{j, i}(x)}
\end{aligned}
$$

Let $\tilde{D}_{i, B}(z)$ denote a four-by-one vector with its $k$-th coordinate being $\tilde{D}_{i, B, k}(z)$ for $k=1,2,3,4$ and $i=1,2$. For any $i$ and $z$, define

$$
M_{B, \gamma}^{i}\left(z, \gamma_{(1, i)}\right)=\gamma_{(1, i)}(x)^{\prime} \tilde{D}_{i, B}(z)
$$

Note $\tilde{D}_{i, B}$ on the right-hand side of $(23)$ is evaluated at the true parameters $\delta_{i}$ and $\gamma_{(1, i)}^{*}$. 
For both $i$, let $\bar{D}_{i, B, k}(x) \equiv E\left[\tilde{D}_{i, B, k}(Z) \mid x\right]$ for all $k$, and $\bar{D}_{i, B, k}^{(s)}(x) \equiv \frac{\partial\left[\bar{D}_{i, B, k}(x) \gamma_{0}^{*}(x)\right]}{\partial X_{s}}$ for $s=1,2$ and $k=3,4$. Define

$$
\begin{aligned}
& \psi_{B, 0}^{i}(x) \equiv \bar{D}_{i, B, 1}(x) \gamma_{0}^{*}(x)-\bar{D}_{i, B, 3}^{(i)}(x)+\mathcal{I}_{B, 1}^{i}(x) \\
& \psi_{B, j}^{i}(x) \equiv \bar{D}_{i, B, 2}(x) \gamma_{0}^{*}(x)-\bar{D}_{i, B, 4}^{(i)}(x)+\mathcal{I}_{B, 2}^{i}(x),
\end{aligned}
$$

where

$$
\begin{aligned}
& \mathcal{I}_{B, 1}^{i}(x) \equiv 1\left\{x_{i}=h_{i}^{*}\left(x_{-i}\right)\right\} \bar{D}_{i, B, 3}(x) \gamma_{0}^{*}(x)-1\left\{x_{i}=l_{i}^{*}\left(x_{-i}\right)\right\} \bar{D}_{i, B, 3}(x) \gamma_{0}^{*}(x) \\
& \mathcal{I}_{B, 2}^{i}(x) \equiv 1\left\{x_{i}=h_{i}^{*}\left(x_{-i}\right)\right\} \bar{D}_{i, B, 4}(x) \gamma_{0}^{*}(x)-1\left\{x_{i}=l_{i}^{*}\left(x_{-i}\right)\right\} \bar{D}_{i, B, 4}(x) \gamma_{0}^{*}(x),
\end{aligned}
$$

with $h_{i}^{*}\left(x_{-i}\right), l_{i}^{*}\left(x_{-i}\right)$ being the supreme and infimum of the support of $X_{i}$ given $x_{-i}$.

For $i=1,2$ and any $h$ twice continuously differentiable in $x_{c}$ over $\Omega_{X}$, define

$$
M_{B, f}^{i}(z ; h) \equiv \frac{C_{i}^{*}(z) \int h\left(t, x_{-i}\right) d t}{\gamma_{0}^{*}(x)}-\frac{C_{i}^{*}(z) h(x) \int \gamma_{0}^{*}\left(t, x_{-i}\right) d t}{\gamma_{0}^{*}(x)^{2}}
$$

where

$$
C_{i}^{*}(z) \equiv \tilde{x}^{\prime}\left[d_{i}-H\left(-x_{i}+\delta_{i} p_{j}^{*}(x)\right)\right]\left[1-\delta_{i} p_{j, i}^{*}(x)\right] .
$$

The functional $M_{B, f}^{i}(z ; h)$ is the Frechet derivative of $m_{B}^{i}\left(z ; \delta_{i}, \gamma_{(1, i)}\right)$ with respect to $\gamma_{(1, i)}$ at $\gamma_{(1, i)}^{*}$ in DGP.Define

$$
\psi_{B, f}^{i}(x) \equiv E\left(\frac{C_{i}^{*}(Z)}{\gamma_{0}^{*}(X)} \mid x_{-i}\right) \gamma_{0}^{*}\left(x_{-i}\right)-\frac{E\left(C_{i}^{*}(Z) \mid x\right)}{\gamma_{0}^{*}(x)} \int \gamma_{0}^{*}\left(t, x_{-i}\right) d t,
$$

where the integral is over the support $\Omega_{X_{i} \mid x_{-i}}$, and we also use $\gamma_{0}^{*}\left(x_{-i}\right)$ to denote the true density of $X_{-i}$. Our proof builds on the observation that $\int M_{B, f}^{i}(z ; h) d F_{Z}^{*}=\int \psi_{B, f}^{i}(x) h(x) d x$.

We now define the major components in the limiting distribution of $\sqrt{G}\left(\hat{\beta}_{i}-\beta_{i}\right)$ :

$$
\begin{aligned}
\tilde{\nu}_{B}^{i}\left(z_{g}\right) & \equiv \psi_{B, 0}^{i}\left(x_{g}\right)+\psi_{B, f}^{i}\left(x_{g}\right)+\psi_{B, j}^{i}\left(x_{g}\right) d_{g, j}-E\left[\psi_{B, 0}^{i}(X)+\psi_{B, f}^{i}(X)+\psi_{B, j}^{i}(X) D_{j}\right] ; \\
\Psi_{\delta}^{i}\left(z_{g}\right) & \equiv-\frac{1}{\rho_{0}}\left[w_{g}\left(m_{\Delta}^{i}\left(x_{g} ; \gamma_{(1, i)}^{*}\right)-\Delta^{i}\right)+\tilde{\nu}_{\Delta}^{i}\left(z_{g}\right)\right] ; \mathcal{M}_{i, \delta}^{*} \equiv E\left[\tilde{D}_{i, B, \delta}\left(Z ; \delta_{i}, \gamma_{(1, i)}^{*}\right)\right] ; \\
\Psi_{B}^{i}(z) & \equiv m_{B}^{i}\left(z ; \delta_{i}, \gamma_{(1, i)}^{*}\right)+\tilde{\nu}_{B}^{i}(z)+\mathcal{M}_{i, \delta}^{*} \Psi_{\delta}^{i}(z) .
\end{aligned}
$$

The key step in finding the limiting distribution of $\sqrt{G}\left(\hat{\beta}_{i}-\beta_{i}\right)$ is to show $\frac{1}{G} \sum_{g} m_{B}^{i}\left(z_{g} ; \hat{\delta}_{i}, \hat{\gamma}_{(1, i)}\right)$ $=\frac{1}{G} \sum_{g} \Psi_{B}^{i}\left(z_{g}\right)+o_{p}\left(G^{-1 / 2}\right)$. Specifically, the difference between $\frac{1}{G} \sum_{g} m_{B}^{i}\left(z_{g} ; \hat{\delta}_{i}, \hat{\gamma}_{(1, i)}\right)$ and the infeasible moment $\frac{1}{G} \sum_{g} m_{B}^{i}\left(z_{g} ; \delta_{i}, \gamma_{(1, i)}^{*}\right)$ is a sample average of some correction terms $\tilde{\nu}_{B}^{i}(z)+$ $\mathcal{M}_{i, \delta}^{*} \Psi_{\delta}^{i}(z)$ plus $o_{p}\left(G^{-\frac{1}{2}}\right)$. The form of these correction terms depends on $\gamma_{(1)}^{*}$ in the DGP as well as how $\hat{\delta}_{i}, \hat{\gamma}_{(1)}$ enter the moment function $m_{B}^{i}$.

Assumption D' (i) For $i=1,2$, the second order derivatives of $m_{B}^{i}\left(z ; \delta_{i}, \gamma_{(1, i)}\right)$ with respect to $\gamma_{(1, i)}(x)$ at $\gamma_{(1, i)}^{*}(x)$ are continuous and bounded over the support of $Z$ (i.e. $\left.\{1,0\}^{2} \otimes \Omega_{X}\right)$. (ii) 
For both $i$ and $s=1,2, \tilde{D}_{i, B}^{(s)}$, are continuous and bounded over $\{1,0\}^{2} \otimes \Omega_{X}$. (iii) For $i=1,2$ and $j=3-i$, there exists $c>0$ such that $E\left[\sup _{\|\eta\| \leq c}\left\|\psi_{B, s}^{i}(x+\eta)\right\|^{4}\right]<\infty$ for $s=0, j$ and $E\left[\sup _{\|\eta\| \leq c}\left\|\psi_{B, f}^{i}(x+\eta)\right\|^{4}\right]<\infty$.

Assumption $\mathbf{W}^{\prime}$ For any $i$ and $x_{-i}$, the support of $X_{i}$ given $x_{-i}$ is convex and closed.

Assumption R' (i) There exists open neighborhoods $\mathcal{N}_{\delta}, \mathcal{N}_{i}$ around $\delta_{i}^{*}, \gamma_{(1, i)}^{*}$ respectively such that $E\left[\sup _{\delta_{i} \in \mathcal{N}_{\delta}, \gamma_{(1, i)} \in \mathcal{N}_{i}}\left\|\tilde{D}_{i, B, \delta}\left(Z ; \delta_{i}, \gamma_{(1, i)}\right)\right\|\right]<\infty$. (ii) $E\left[\left\|\Psi_{B}^{i}(Z)-\tilde{X}^{\prime} \tilde{X} \beta_{i}\right\|^{2}\right]<+\infty$. (iii) $E\left[\tilde{X}^{\prime} \tilde{X}\right]$ is non-singular.

Condition R'-(i) is used for showing the difference between $\frac{1}{G} \sum_{g} m_{B}^{i}\left(z_{g} ; \hat{\delta}_{i}, \hat{\gamma}_{(1, i)}\right)$ and $\frac{1}{G} \sum_{g} m_{B}^{i}\left(z_{g}\right.$; $\left.\delta_{i}, \hat{\gamma}_{(1, i)}\right)$ is represented by a sample average of a certain function plus an $o_{p}\left(G^{-\frac{1}{2}}\right)$ term. Under R'(i), this function takes the form of the product of $E\left[\tilde{D}_{i, B, \delta}\left(z ; \delta_{i}, \gamma_{(1, i)}^{*}\right)\right]$ and the influence function that leads to the limiting distribution of $\sqrt{G}\left(\hat{\delta}_{i}-\delta_{i}\right)$.

Similar to the case with $\hat{\Delta}^{i}$, we can apply the linearization argument and the V-statistic Projection Theorem to show that, under conditions in D'-(i) and (ii), $\frac{1}{G} \sum_{g}\left[m_{B}^{i}\left(x_{g} ; \delta_{i}, \hat{\gamma}_{(1)}\right)\right.$ $\left.m_{B}^{i}\left(x_{g} ; \delta_{i}, \gamma_{(1)}^{*}\right)\right]$ can be written as $\int M_{B, \gamma}^{i}\left(z, \hat{\gamma}_{(1, i)}-\gamma_{(1, i)}^{*}\right)+M_{B, f}^{i}\left(z ; \hat{\gamma}_{0}-\gamma_{0}^{*}\right) d F_{Z}^{*}$ plus $o_{p}\left(G^{-\frac{1}{2}}\right)$, where $F_{Z}^{*}$ is the true distribution of $Z$ in the DGP. Again by definition and smoothness properties in S', for any $\gamma_{(1, i)}$ that is twice continuously differentiable in excluded regressors, the functions $\psi_{B, 0}^{i}, \psi_{B, j}^{i}$ and $\psi_{B, f}^{i}$ can be shown to satisfy:

$$
\int M_{B, \gamma}^{i}\left(z ; \gamma_{(1, i)}\right)+M_{B, f}^{i}\left(z ; \gamma_{0}\right) d F_{Z}^{*}=\int\left[\psi_{B, 0}^{i}(x)+\psi_{B, f}^{i}(x)\right] \gamma_{0}(x)+\psi_{B, j}^{i}(x) \gamma_{j}(x) d x
$$

using an argument of integration by parts.

Using this equation, the difference $\int M_{B, \gamma}^{i}\left(z ; \hat{\gamma}_{(1, i)}-\gamma_{(1, i)}^{*}\right)+M_{B, f}^{i}\left(z ; \hat{\gamma}_{(1, i)}-\gamma_{(1, i)}^{*}\right) d F_{Z}^{*}$ can be expressed as $\int \tilde{\nu}_{B}^{i}(z) d \tilde{F}_{Z}$, where $\tilde{\nu}_{B}^{i}(z)$ was defined above and $\tilde{F}_{Z}$ is some smoothed version of the empirical distribution of $Z$ as mentioned in the proof of asymptotic properties of $\hat{\delta}_{i}$. Condition D'-(iii) is then used for showing the difference between $\int \tilde{\nu}_{B}^{i}(z) d \tilde{F}_{Z}$ and $\frac{1}{G} \sum_{g} \tilde{\nu}_{B}^{i}\left(z_{g}\right)$ is $o_{p}\left(G^{-\frac{1}{2}}\right)$. Thus the difference between $\frac{1}{G} \sum_{g} m_{B}^{i}\left(z_{g} ; \delta_{i}, \hat{\gamma}_{(1)}\right)$ and $\frac{1}{G} \sum_{g}\left[m_{B}^{i}\left(z_{g} ; \delta_{i}, \gamma_{(1)}^{*}\right)+\tilde{\nu}_{B}^{i}\left(z_{g}\right)\right]$ is $o_{p}\left(G^{-1 / 2}\right)$. For D'-(iii) to hold, it suffices to have that, for $i=1,2$, there exists $c_{i}>0$ such that $E\left[\sup _{\|\eta\| \leq c_{i}}\right.$ $\left.\left\|\bar{D}_{i, B}(x+\eta)\right\|^{4}\right]<\infty$ and $E\left[\sup _{\|\eta\| \leq c_{i}}\left\|\bar{D}_{i, B}^{(s)}(x+\eta)\right\|^{4}\right]<\infty$. Condition R'-(ii) ensures the Central Limit Theorem can be applied. Condition R'-(iii) is the standard full-rank condition necessary for consistency of regressor estimators. Proof of the following theorem is included in the supplement of this paper.

Theorem 5 Under $A 1-4, A 5^{\prime \prime}$ and $S^{\prime}, K, B, D^{\prime}, W^{\prime}, R, \sqrt{G}\left(\hat{\beta}_{i}-\beta_{i}\right) \stackrel{d}{\rightarrow} N\left(0, \Sigma_{B}^{i}\right)$ for $i=1,2$, where

$$
\Sigma_{B}^{i} \equiv\left[E\left(\tilde{X}^{\prime} \tilde{X}\right)^{-1}\right] \operatorname{Var}\left(\Psi_{B}^{i}(Z)-\tilde{X}^{\prime} \tilde{X} \beta_{i}\right)\left[E\left(\tilde{X}^{\prime} \tilde{X}\right)^{-1}\right]^{\prime}
$$


Estimation errors in $\hat{\delta}_{i}$ and $\hat{p}_{j}$ affect the distribution of $\hat{\beta}_{i}$ in general. The optimal rate of convergence of $\hat{p}_{j}$ is generally slower than $\sqrt{G}$ because $\hat{p}_{j}$ depends on the number of continuous coordinates in $X$, but $\hat{\beta}_{i}$ can still converge at the parametric rate because $\hat{\beta}_{i}$ takes the form of a sample average.

\subsection{Discussion of $\beta_{i}$ Estimation Rates}

To obtain a parametric convergence rate for $\hat{\beta}_{i}$, Assumption S' assumes that excluded regressors have bounded support, and that support of $V_{i}$ given some $x_{-i}=\left(x_{e,-i}, \tilde{x}\right)$ includes that of $-\tilde{x} \beta_{i}+\epsilon_{i}$ given $\tilde{x}$. This necessarily requires the support of $\epsilon_{i}$ given $\tilde{x}$ to be bounded. As discussed earlier, such boundedness is a sensible assumption in the entry game example.

Lewbel (2000) shows that special regressor estimates can converge at parametric rates $(\sqrt{G}$ in the current context) with unbounded support, but doing so requires that that the special regressor have very thick tails. It should therefore be possible to relax the bounded support restriction in Assumption S'. However, Khan and Tamer's (2010) impossibility result shows that, in the unboundedness case, the tails would need to be thick enough to give the special regressor infinite variance. ${ }^{10}$ Moreover our estimator would need to be modified to incorporate asymptotic trimming or some similar device to deal with the denominator of $Y_{i}$ going to zero in the tails.

More generally, without bounded support the rate of convergence of $\hat{\beta}_{i}$ depends on the tail behavior of the distributions of $V_{i}$ given $x_{-i}$. Robust inference of $\hat{\beta}_{i}$ independent of the tail behaviors might be conducted in our context using a "rate-adaptive" approach as discussed in Andrews and Schafgans (1993) and Khan and Tamer (2010). This would involve performing inference on a studentized version of $\hat{\beta}_{i}$. More speculatively, it may also be possible to attain parametric rates of convergence without these tail thickness constraints by adapting some version of tail symmetry as in Magnac and Maurin (2007) to our game context. We leave these topics to future research.

\section{Monte Carlo Simulations}

In this section we present evidence for the performance of the estimators in 2-by-2 entry games with linear payoffs. For $i=1,2$, the payoff for Firm $i$ from entry $\left(D_{i}=1\right)$ is $\beta_{i}^{0}+\beta_{i}^{1} \tilde{X}-X_{i}+\delta_{i} D_{3-i}-\epsilon_{i}$,

\footnotetext{
${ }^{10}$ More precisely, Khan and Tamer (2010) showed for a single-agent, binary regression with a special regressor that the semiparametric efficiency bound for linear coefficients are not finite as long as the second moment of all regressors (including the special regressors) are finite. For a further simplified model where $Y=1\{\alpha+v+\varepsilon \geq 0\}$ (with $v \perp \varepsilon, \alpha$ constant and both $v, \varepsilon$ distributed over the real-line), they showed that the rate of convergence for an inverse-density-weighted estimator can vary between $N^{-1 / 4}$ and the parametric rate, depending on the relative tail behaviors.
} 
where $D_{3-i}$ is the entry decision of $i$ 's competitor. The payoff from staying out is 0 for both $i=1,2$. The non-excluded regressor $\tilde{X}$ is discrete with $\operatorname{Pr}(\tilde{X}=1 / 2)=\operatorname{Pr}(\tilde{X}=1)=1 / 2$. The true parameters in the data-generating process (DGP) are set to $\left[\beta_{1}^{0}, \beta_{1}^{1}\right]=[1.8,0.5],\left[\beta_{2}^{0}, \beta_{2}^{1}\right]=[1.6,0.8]$ and $\left[\delta_{1}, \delta_{2}\right]=[-1.3,-1.3]$ For both $i=1,2$, the support of the observable part of the fixed costs (i.e. excluded regressors $X_{i}$ ) is $[0,5]$ and the supports for the unobservable part of the fixed costs (i.e. $\left.\epsilon_{i}\right)$ is $[-2,2]$. All variables $\left(\tilde{X}, X_{1}, X_{2}, \epsilon_{1}, \epsilon_{2}\right)$ are mutually independent. To illustrate how the semiparametric estimator performs under various distributions of unobservable states, we experiment with two DGP designs: one in which both $X_{i}$ and $\epsilon_{i}$ are uniformly distributed (Uniform Design); and one in which $X_{i}$ is distributed with symmetric, bell-shaped density $f_{X_{i}}(t)=\frac{3}{8}\left(1-\left(\frac{2 t}{5}-\right.\right.$ $\left.1)^{2}\right)^{2}$ over $[0,5]$ while $\epsilon_{i}$ is distributed with symmetric, bell-shaped density $f_{\epsilon_{i}}(t)=\frac{15}{32}\left(1-\frac{t^{2}}{4}\right)^{2}$ over $[-2,2]$. That is, both $X_{i}, \epsilon_{i}$ are linear transformation of a random variable whose density is given by the Quartic (Bi-weight) kernel, so we will refer to the second design as the $B W K$ design. By construction, the conditional independence, additive separability, large support, and monotonicity conditions are all satisfied by these designs.

For each design, we simulate $S=300$ samples and calculate summary statistics from empirical distributions of estimators $\hat{\delta}_{i}$ from these samples. These statistics include the mean, standard deviation (Std.Dev), $25 \%$ percentile $(L Q)$, median, $75 \%$ percentile $(H Q)$, root of mean squared error $(R M S E)$ and median of absolute error $(M A E)$. Both $R M S E$ and $M A E$ are estimated using the empirical distribution of estimators and the knowledge of our true parameters in the design.

Table 1(a) reports performance of $\left(\hat{\delta}_{1}, \hat{\delta}_{2}\right)$ under the uniform design. The two statistics reported in each cell of Table 1(a) correspond to those of $\left[\hat{\delta}_{1}, \hat{\delta}_{2}\right]$ respectively. Each row of Table 1(a) reports Each row relates to a different sample size $G$ (either 5,000 or 10,000) and certain choices of bandwidth $\sigma$ for estimating $p_{i}^{*}, p_{j}^{*}$ and their partial derivatives w.r.t. $X_{i}, X_{j}$. We use the tri-weight kernel function (i.e. $\left.K(t)=\frac{35}{32}\left(1-t^{2}\right)^{3} 1\{|t| \leq 1\}\right)$ in estimation. We choose the bandwidth (b.w.) through cross-validation by minimizing the Expectation of Average Squared Error (MASE) in the estimation of $p_{i}^{*}$ and $p_{j}^{*}$. This is done by choosing the bandwidths that minimize the Estimated Predication Error (EPE) for estimating $p_{i}^{*}, p_{j}^{*} .{ }^{11}$ To study how robust the performance of our estimator is against various bandwidths, we report summary statistics for our estimator under both intentional under-smoothing (with a bandwidth equal to half of b.w.) and over-smoothing (with a bandwidth equal to 1.5 times of b.w.). To implement our estimator, we estimate the set of states $\omega$ satisfying NDS by $\left\{x: \hat{p}_{i}(x), \hat{p}_{j}(x) \in(0,1), \hat{p}_{i, i}(x), \hat{p}_{j, j}(x) \neq 0\right.$ and $\left.\hat{p}_{i, i}(x) \hat{p}_{j, j}(x) \neq \hat{p}_{i, j}(x) \hat{p}_{j, i}(x)\right\}$.

\footnotetext{
${ }^{11}$ See Page 119 of Pagan and Ullah (1999) for definition of EPE and MASE.
} 
Table 1(a): Estimator for $\left(\delta_{1}, \delta_{2}\right)$ (Uniform Design)

\begin{tabular}{|c|c|ccccccc|}
\hline$G$ & & Mean & Std.Dev. & LQ & Median & HQ & RMSE & MAE \\
\hline \hline \multirow{3}{*}{$5 k$} & b.w. & {$[-1.397,-1.384]$} & {$[0.163,0.174]$} & {$[-1.514,-1.499]$} & {$[-1.387,-1.386]$} & {$[-1.294,-1.267]$} & {$[0.189,0.193]$} & {$[0.123,0.122]$} \\
& $\frac{1}{2}$ b.w. & {$[-1.430,-1.433]$} & {$[0.282,0.292]$} & {$[-1.606,-1.621]$} & {$[-1.408,-1.404]$} & {$[-1.257,-1.232]$} & {$[0.310,0.320]$} & {$[0.196,0.199]$} \\
& $\frac{3}{2}$ b.w. & {$[-1.442,-1.429]$} & {$[0.141,0.148]$} & {$[-1.536,-1.529]$} & {$[-1.436,-1.425]$} & {$[-1.344,-1.324]$} & {$[0.200,0.196]$} & {$[0.144,0.139]$} \\
\hline \hline \multirow{3}{*}{$10 k$} & b.w. & {$[-1.380,-1.379]$} & {$[0.113,0.119]$} & {$[-1.456,-1.457]$} & {$[-1.381,-1.374]$} & {$[-1.298,-1.294]$} & {$[0.138,0.143]$} & {$[0.096,0.098]$} \\
& $\frac{1}{2}$ b.w. & {$[-1.390,-1.394]$} & {$[0.187,0.185]$} & {$[-1.519,-1.520]$} & {$[-1.376,-1.386]$} & {$[-1.256,-1.254]$} & {$[0.207,0.207]$} & {$[0.134,0.129]$} \\
& $\frac{3}{2}$ b.w. & {$[-1.424,-1.420]$} & {$[0.098,0.102]$} & {$[-1.488,-1.487]$} & {$[-1.421,-1.417]$} & {$[-1.352,-1.349]$} & {$[0.158,0.157]$} & {$[0.121,0.117]$} \\
\hline
\end{tabular}

The column of RMSE in Table 1(a) shows that the mean squared error (MSE) in estimation diminishes as the sample size increases from $G=5000$ to $G=10000$. There is also evidence for improvement of the estimator in terms median absolute errors as $G$ increases. The trade-off between variance and bias of the estimator as bandwidth changes is also evident from the first two columns in Table 1(a). The choice of bandwidth has a moderate effect on estimator performance.

Table 1(b): Estimator for $\left(\delta_{1}, \delta_{2}\right)(B W K$ Design $)$

\begin{tabular}{|c|c|ccccccc|}
\hline \multirow{2}{*}{$G$} & & Mean & Std.Dev. & LQ & Median & $H Q$ & RMSE & MAE \\
\hline \hline \multirow{5}{*}{$5 k$} & b.w. & {$[-1.347,-1.342]$} & {$[0.095,0.090]$} & {$[-1.402,-1.410]$} & {$[-1.343,-1.335]$} & {$[-1.287,-1.282]$} & {$[0.105,0.100]$} & {$[0.071,0.062]$} \\
& $\frac{1}{2}$ b.w. & {$[-1.343,-1.343]$} & {$[0.120,0.112]$} & {$[-1.418,-1.424]$} & {$[-1.342,-1.345]$} & {$[-1.263,-1.266]$} & {$[0.127,0.120]$} & {$[0.084,0.081]$} \\
& $\frac{3}{2}$ b.w. & {$[-1.406,-1.440]$} & {$[0.092,0.090]$} & {$[-1.462,-1.464]$} & {$[-1.404,-1.391]$} & {$[-1.346,-1.344]$} & {$[0.141,0.135]$} & {$[0.108,0.095]$} \\
\hline \hline \multirow{5}{*}{$10 k$} & b.w. & {$[-1.317,-1.320]$} & {$[0.061,0.061]$} & {$[-1.361,-1.356]$} & {$[-1.312,-1.314]$} & {$[-1.276,-1.276]$} & {$[0.063,0.064]$} & {$[0.042,0.038]$} \\
& $\frac{1}{2}$ b.w. & {$[-1.309,-1.311]$} & {$[0.076,0.077]$} & {$[-1.361,-1.357]$} & {$[-1.305,-1.300]$} & {$[-1.264,-1.263]$} & {$[0.076,0.077]$} & {$[0.048,0.045]$} \\
& $\frac{3}{2}$ b.w. & {$[-1.365,-1.368]$} & {$[0.058,0.060]$} & {$[-1.407,-1.404]$} & {$[-1.361,-1.365]$} & {$[-1.325,-1.325]$} & {$[0.087,0.090]$} & {$[0.061,0.066]$} \\
\hline
\end{tabular}

Table 1(b) reports the same statistics for the BWK design where both $X_{i}, \epsilon_{i}$ are distributed with bell-shaped densities. Again there is strong evidence for convergence of the estimator in terms of MSE (and MAE). A comparison between panels (a) and (b) in Table 1 suggests the estimators for $\delta_{i}, \delta_{j}$ perform better under the BWK design. First, for a given sample size, the RMSE is smaller for the BWK design. Second, as sample size increases, improvement of performance (as measured by percentage reductions in RMSE) is larger for the BWK design. This difference is due in part to the fact that, for a given sample size, the BWK design puts less probability mass towards tails of the density of $X_{i}$. To see this, note that by construction, the large support property of the model implies $p_{i}, p_{j}$ could hit the boundaries (0 and 1) for large or small values of $X_{i}, X_{j}$ in the tails. Therefore states with such extreme values of $x_{i}, x_{j}$ are likely to be trimmed as we construct $\hat{\delta}_{i}, \hat{\delta}_{j}$ conditioning on states in $\omega$ (i.e. those satisfying the NDS condition). The uniform distribution assigns higher probability mass towards the tails than bi-weight kernel densities. Thus, for a fixed $N$, the uniform design tends to trim away more observations than BWK design while estimating $\delta_{i}, \delta_{j}$. Note that while these trimmed out tail observations do not contribute towards estimation of $\hat{\delta}_{i}, \hat{\delta}_{j}$, their presence is required for parametric rate convergence of $\hat{\beta}_{i}^{0}, \hat{\beta}_{i}^{1}$. 
Next, we report performance of estimators $\left(\hat{\beta}_{i}^{0}, \hat{\beta}_{i}^{1}\right)_{i=1,2}$ in Table 2, where we experiment with the same set of bandwidths as in Table 1. Besides, we report in Table 2 the performance of an "infeasible version" of our estimator where the preliminary estimates for $p_{i}, p_{j}$ and their partial derivatives w.r.t. excluded regressors are replaced by true values in the DGP. Panels (a) and (b) show summary statistics from the estimates in $S=300$ simulated samples under the uniform design.

Table 2(a): Estimator for $\left(\beta_{i}^{0}, \beta_{i}^{1}\right)$ (Uniform Design, $G=5 k$ )

\begin{tabular}{|c|c|ccccccc|}
\hline & & Mean & Std.Dev. & $L Q$ & Median & $H Q$ & RMSE & MAE \\
\hline \hline Infeasible & {$\left[\hat{\beta}_{1}^{0}, \hat{\beta}_{1}^{1}\right]$} & {$[1.818,0.487]$} & {$[0.088,0.116]$} & {$[1.756,0.408]$} & {$[1.819,0.483]$} & {$[1.879,0.564]$} & {$[0.090,0.117]$} & {$[0.062,0.081]$} \\
& {$\left[\hat{\beta}_{2}^{0}, \hat{\beta}_{2}^{1}\right]$} & {$[1.617,0.786]$} & {$[0.081,0.105]$} & {$[1.560,0.710]$} & {$[1.622,0.785]$} & {$[1.677,0.855]$} & {$[0.083,0.105]$} & {$[0.060,0.073]$} \\
\hline \hline b.w. & {$\left[\hat{\beta}_{1}^{0}, \hat{\beta}_{1}^{1}\right]$} & {$[1.835,0.510]$} & {$[0.095,0.120]$} & {$[1.774,0.427]$} & {$[1.839,0.502]$} & {$[1.900,0.599]$} & {$[0.101,0.120]$} & {$[0.066,0.085]$} \\
& {$\left[\hat{\beta}_{2}^{0}, \hat{\beta}_{2}^{1}\right]$} & {$[1.636,0.801]$} & {$[0.096,0.111]$} & {$[1.571,0.713]$} & {$[1.635,0.801]$} & {$[1.706,0.884]$} & {$[0.102,0.111]$} & {$[0.079,0.085]$} \\
\hline \hline$\frac{1}{2} b . w$. & {$\left[\hat{\beta}_{1}^{0}, \hat{\beta}_{1}^{1}\right]$} & {$[1.875,0.490]$} & {$[0.104,0.123]$} & {$[1.816,0.403]$} & {$[1.873,0.482]$} & {$[1.946,0.574]$} & {$[0.128,0.123]$} & {$[0.088,0.090]$} \\
& {$\left[\hat{\beta}_{2}^{0}, \hat{\beta}_{2}^{1}\right]$} & {$[1.685,0.770]$} & {$[0.107,0.114]$} & {$[1.604,0.682]$} & {$[1.689,0.770]$} & {$[1.764,0.853]$} & {$[0.136,0.117]$} & {$[0.102,0.086]$} \\
\hline \hline$\frac{3}{2}$ b.w. & {$\left[\hat{\beta}_{1}^{0}, \hat{\beta}_{1}^{1}\right]$} & {$[1.815,0.533]$} & {$[0.095,0.123]$} & {$[1.751,0.446]$} & {$[1.812,0.523]$} & {$[1.887,0.620]$} & {$[0.096,0.127]$} & {$[0.068,0.089]$} \\
& {$\left[\hat{\beta}_{2}^{0}, \hat{\beta}_{2}^{1}\right]$} & {$[1.613,0.826]$} & {$[0.097,0.112]$} & {$[1.544,0.737]$} & {$[1.617,0.826]$} & {$[1.684,0.911]$} & {$[0.098,0.115]$} & {$[0.074,0.079]$} \\
\hline
\end{tabular}

Table 2(b): Estimator for $\left(\beta_{i}^{0}, \beta_{i}^{1}\right)$ (Uniform Design, $G=10 k$ )

\begin{tabular}{|c|c|ccccccc|}
\hline & & Mean & Std.Dev. & $L Q$ & Median & $H Q$ & RMSE & MAE \\
\hline \hline \multirow{2}{*}{ Infeasible } & {$\left[\hat{\beta}_{1}^{0}, \hat{\beta}_{1}^{1}\right]$} & {$[1.816,0.492]$} & {$[0.059,0.076]$} & {$[1.777,0.440]$} & {$[1.811,0.496]$} & {$[1.855,0.542]$} & {$[0.061,0.076]$} & {$[0.040,0.052]$} \\
& {$\left[\hat{\beta}_{2}^{0}, \hat{\beta}_{2}^{1}\right]$} & {$[1.627,0.776]$} & {$[0.063,0.078]$} & {$[1.587,0.725]$} & {$[1.629,0.773]$} & {$[1.667,0.826]$} & {$[0.069,0.081]$} & {$[0.046,0.051]$} \\
\hline \hline b.w. & {$\left[\hat{\beta}_{1}^{0}, \hat{\beta}_{1}^{1}\right]$} & {$[1.823,0.525]$} & {$[0.067,0.083]$} & {$[1.779,0.477]$} & {$[1.822,0.531]$} & {$[1.866,0.577]$} & {$[0.071,0.087]$} & {$[0.047,0.059]$} \\
& {$\left[\hat{\beta}_{2}^{0}, \hat{\beta}_{2}^{1}\right]$} & {$[1.631,0.811]$} & {$[0.073,0.085]$} & {$[1.584,0.754]$} & {$[1.634,0.809]$} & {$[1.676,0.864]$} & {$[0.079,0.086]$} & {$[0.056,0.053]$} \\
\hline \hline$\frac{1}{2}$ b.w. & {$\left[\hat{\beta}_{1}^{0}, \hat{\beta}_{1}^{1}\right]$} & {$[1.847,0.497]$} & {$[0.069,0.082]$} & {$[1.801,0.451]$} & {$[1.849,0.500]$} & {$[1.888,0.548]$} & {$[0.083,0.081]$} & {$[0.057,0.049]$} \\
& {$\left[\hat{\beta}_{2}^{0}, \hat{\beta}_{2}^{1}\right]$} & {$[1.658,0.780]$} & {$[0.074,0.085]$} & {$[1.611,0.730]$} & {$[1.658,0.782]$} & {$[1.700,0.831]$} & {$[0.094,0.087]$} & {$[0.067,0.059]$} \\
\hline \hline$\frac{3}{2}$ b.w. & {$\left[\hat{\beta}_{1}^{0}, \hat{\beta}_{1}^{1}\right]$} & {$[1.790,0.575]$} & {$[0.070,0.087]$} & {$[1.745,0.522]$} & {$[1.792,0.582]$} & {$[1.837,0.631]$} & {$[0.071,0.115]$} & {$[0.049,0.090]$} \\
& {$\left[\hat{\beta}_{2}^{0}, \hat{\beta}_{2}^{1}\right]$} & {$[1.599,0.860]$} & {$[0.076,0.089]$} & {$[1.548,0.803]$} & {$[1.604,0.858]$} & {$[1.644,0.918]$} & {$[0.075,0.107]$} & {$[0.049,0.070]$} \\
\hline
\end{tabular}

Similar to Table 1, these panels in Table 2 show some evidence that estimators are converging (with MSE diminishing) as sample size increases. In Table 2(a) and 2(b), the choices of bandwidth now seem to have less impact on estimator performance relative to Table 1 . Furthermore, the trade-off between bias and variances of $\hat{\beta}_{i}^{0}, \hat{\beta}_{i}^{1}$ as bandwidth varies is also less evident than in Table 1. This may be partly explained by the fact that the first-stage estimates $\hat{\delta}_{i}$ and $\hat{\delta}_{j}$ (which itself is a sample moment involving preliminary estimates of nuisance parameters $\hat{p}_{i}, \hat{p}_{j}, \hat{p}_{i, j}, \hat{p}_{j, i}$ etc) now enter $\hat{\beta}_{1}^{0}, \hat{\beta}_{1}^{1}$ through sample moments. While estimating $\beta_{i}^{0}, \beta_{i}^{1}$, these moments are then averaged over all observed states in data, including those not necessarily satisfying NDS. This second-round averaging, which involves more observations from data, possibly further mitigates the impact of bandwidths for nuisance estimates $\left(\hat{p}_{i}, \hat{p}_{j}\right.$ etc) on final estimates. It is also worth noting that the performance of the feasible estimator is somewhat comparable to that of the infeasible estimators in 
terms of MSE and MAE. Again, this is as expected, because the final estimator for $\beta_{i}^{0}, \beta_{i}^{1}$ requires further averaging of sample moments that involve preliminary estimates.

Table 2(c): Estimator for $\left(\beta_{i}^{0}, \beta_{i}^{1}\right)$ (BWK Design, $G=5 k$ )

\begin{tabular}{|c|c|ccccccc|}
\hline & & Mean & Std.Dev. & LQ & Median & $H Q$ & RMSE & MAE \\
\hline \hline \multirow{2}{*}{ Infeasible } & {$\left[\hat{\beta}_{1}^{0}, \hat{\beta}_{1}^{1}\right]$} & {$[1.811,0.513]$} & {$[0.072,0.095]$} & {$[1.757,0.448]$} & {$[1.813,0.516]$} & {$[1.860,0.573]$} & {$[0.072,0.096]$} & {$[0.051,0.064]$} \\
& {$\left[\hat{\beta}_{2}^{0}, \hat{\beta}_{2}^{1}\right]$} & {$[1.618,0.804]$} & {$[0.064,0.084]$} & {$[1.574,0.747]$} & {$[1.620,0.801]$} & {$[1.661,0.858]$} & {$[0.066,0.083]$} & {$[0.046,0.055]$} \\
\hline \hline b.w. & {$\left[\hat{\beta}_{1}^{0}, \hat{\beta}_{1}^{1}\right]$} & {$[1.813,0.513]$} & {$[0.074,0.096]$} & {$[1.773,0.456]$} & {$[1.814,0.514]$} & {$[1.859,0.576]$} & {$[0.075,0.097]$} & {$[0.045,0.055]$} \\
& {$\left[\hat{\beta}_{2}^{0}, \hat{\beta}_{2}^{1}\right]$} & {$[1.619,0.797]$} & {$[0.069,0.085]$} & {$[1.569,0.739]$} & {$[1.618,0.795]$} & {$[1.665,0.854]$} & {$[0.071,0.085]$} & {$[0.051,0.058]$} \\
\hline \hline$\frac{1}{2}$ b.w. & {$\left[\hat{\beta}_{1}^{0}, \hat{\beta}_{1}^{1}\right]$} & {$[1.835,0.487]$} & {$[0.073,0.097]$} & {$[1.784,0.421$} & {$[1.835,0.494]$} & {$[1.881,0.544]$} & {$[0.081,0.098]$} & {$[0.058,0.061]$} \\
& {$\left[\hat{\beta}_{2}^{0}, \hat{\beta}_{2}^{1}\right]$} & {$[1.641,0.776]$} & {$[0.073,0.089]$} & {$[1.593,0.716]$} & {$[1.643,0.774]$} & {$[1.688,0.831]$} & {$[0.083,0.092]$} & {$[0.061,0.066]$} \\
\hline \hline$\frac{3}{2}$ b.w. & {$\left[\hat{\beta}_{1}^{0}, \hat{\beta}_{1}^{1}\right]$} & {$[1.796,0.574]$} & {$[0.074,0.098]$} & {$[1.742,0.503]$} & {$[1.796,0.576]$} & {$[1.848,0.637]$} & {$[0.074,0.123]$} & {$[0.053,0.090]$} \\
& {$\left[\hat{\beta}_{2}^{0}, \hat{\beta}_{2}^{1}\right]$} & {$[1.619,0.835]$} & {$[0.071,0.088]$} & {$[1.564,0.775]$} & {$[1.620,0.832]$} & {$[1.665,0.892]$} & {$[0.074,0.095]$} & {$[0.053,0.056]$} \\
\hline
\end{tabular}

Table 2(d): Estimator for $\left(\beta_{i}^{0}, \beta_{i}^{1}\right)$ (BWK Design, $\left.G=10 k\right)$

\begin{tabular}{|c|c|ccccccc|}
\hline & & Mean & Std.Dev. & LQ & Median & $H Q$ & RMSE & MAE \\
\hline \hline \multirow{2}{*}{ Infeasible } & {$\left[\hat{\beta}_{1}^{0}, \hat{\beta}_{1}^{1}\right]$} & {$[1.805,0.521]$} & {$[0.052,0.067]$} & {$[1.778,0.484]$} & {$[1.811,0.523]$} & {$[1.842,0.561]$} & {$[0.052,0.070]$} & {$[0.030,0.044]$} \\
& {$\left[\hat{\beta}_{2}^{0}, \hat{\beta}_{2}^{1}\right]$} & {$[1.616,0.809]$} & {$[0.053,0.062]$} & {$[1.587,0.768]$} & {$[1.619,0.803]$} & {$[1.649,0.847]$} & {$[0.055,0.062]$} & {$[0.038,0.039]$} \\
\hline \hline b.w. & {$\left[\hat{\beta}_{1}^{0}, \hat{\beta}_{1}^{1}\right]$} & {$[1.810,0.507]$} & {$[0.052,0.067]$} & {$[1.773,0.469]$} & {$[1.812,0.506]$} & {$[1.845,0.552]$} & {$[0.053,0.067]$} & {$[0.037,0.046]$} \\
& {$\left[\hat{\beta}_{2}^{0}, \hat{\beta}_{2}^{1}\right]$} & {$[1.618,0.796]$} & {$[0.052,0.065]$} & {$[1.581,0.749]$} & {$[1.620,0.793]$} & {$[1.654,0.838]$} & {$[0.055,0.065]$} & {$[0.038,0.044]$} \\
\hline \hline$\frac{1}{2} b . w$. & {$\left[\hat{\beta}_{1}^{0}, \hat{\beta}_{1}^{1}\right]$} & {$[1.827,0.487]$} & {$[0.054,0.070]$} & {$[1.789,0.442]$} & {$[1.828,0.490]$} & {$[1.865,0.532]$} & {$[0.060,0.071]$} & {$[0.042,0.045]$} \\
& {$\left[\hat{\beta}_{2}^{0}, \hat{\beta}_{2}^{1}\right]$} & {$[1.632,0.780]$} & {$[0.055,0.070]$} & {$[1.592,0.735]$} & {$[1.635,0.781]$} & {$[1.670,0.825]$} & {$[0.063,0.070]$} & {$[0.043,0.050]$} \\
\hline \hline$\frac{3}{2} b . w$. & {$\left[\hat{\beta}_{1}^{0}, \hat{\beta}_{1}^{1}\right]$} & {$[1.808,0.527]$} & {$[0.056,0.067]$} & {$[1.766,0.489]$} & {$[1.808,0.527]$} & {$[1.841,0.571]$} & {$[0.056,0.072]$} & {$[0.036,0.050]$} \\
& {$\left[\hat{\beta}_{2}^{0}, \hat{\beta}_{2}^{1}\right]$} & {$[1.619,0.807]$} & {$[0.052,0.064]$} & {$[1.582,0.764]$} & {$[1.621,0.804]$} & {$[1.655,0.849]$} & {$[0.055,0.065]$} & {$[0.040,0.043]$} \\
\hline
\end{tabular}

The remaining two panels 2(c) and 2(d) report estimator performance for the BWK design. These two panels exhibit similar patterns as noted for Table 2(a) and 2(b). The performance of $\left(\hat{\beta}_{i}^{0}, \hat{\beta}_{i}^{1}\right)$ under the BWK design is slightly better than that in the uniform design in terms of both MSE and MAE. The uniform design has more observations in the data that are closer to the boundary of supports than the BWK, which, for a given accuracy of first stage estimates, should have made estimation of $\beta^{0}, \beta_{i}^{1}$ more accurate under the uniform design. However, this effect was offset by the impact of distributions of $X_{i}, \epsilon_{i}$ on first-stage estimators $\hat{\delta}_{i}, \hat{\delta}_{j}$, which were more precisely estimated in the uniform design.

\section{Extension: Multiple Bayesian Nash Equilibria}

As discussed in Section 2, the model may admit multiple BNE in general. Preceding sections maintained the assumption that choices under each state are generated by a single BNE in the 
data-generating process (A2). This section shows how to extend our identification and estimation strategies to allow for multiple BNE in some states.

Our approach differs qualitatively from other methods such as those surveyed in the introduction, and only requires fairly mild nonparametric restrictions. Consider the possibly unknown set of all states in which choices observed in data are rationalized by a single BNE. Denote this set by $\omega^{*} \subseteq \Omega_{X}$. Note $\omega^{*}$ not only includes those states in which the system of equations in (1) has a unique solution, but also includes those in which (1) admits multiple solutions in $\left\{s_{i}(x, .)\right\}_{i \in N}$ but for which some (possibly unknown to the researcher) equilibrium selection mechanism in data is degenerate at only one of them.

We first modify previous arguments to identify $\alpha_{i}$ and interaction effects $\delta_{i}(\tilde{x})$ by conditioning on states being in a known subset $\omega^{\prime}$ of $\omega^{*}$. Furthermore, provided the excluded regressors $X_{e}$ vary sufficiently over $\omega^{\prime}$, we similarly extend previous arguments to identify the baseline payoffs. These extensions of results in Sections 3.2 and 3.4 are provided in Section 8.1. Also, in Appendix B we provide sufficient conditions to guarantee that a non-empty set $\omega^{\prime}$ exists which contains enough states, and hence is sufficiently large enough, to allow identification of players' payoffs in the model.

Implementation of this identification strategy assumes knowledge of some sufficiently large subset $\omega^{\prime}$ of $\omega^{*}$. Our final results show that this assumption is testable, that is, given a sufficiently large candidate set of states $\bar{\omega}$, we can use our data to test if $\bar{\omega}$ is a subset of $\omega^{*}$, and hence test if $\bar{\omega}$ is a valid choice of a set of states $\omega^{\prime}$ to use in our identification method.

\subsection{Identification under multiple equilibria in some states}

Corollary 4 (Theorem 1)Suppose A1,3 hold and let $\omega^{\prime}$ be an open subset of $\omega^{*}$ that satisfies NDS. Then (i) $\left(\alpha_{1}, ., \alpha_{N}\right)$ is identified as in (7) in Theorem 1 with $\omega$ replaced by $\omega^{\prime}$. (ii) For all $\tilde{x}$ such that $\operatorname{Pr}\left(X \in \omega^{\prime} \mid \tilde{X}=\tilde{x}\right)>0,\left(\delta_{1}(\tilde{x}), ., \delta_{N}(\tilde{x})\right)$ is identified as in (8) in Theorem 1 with $\omega$ replaced by $\omega^{\prime}$.

Corollary 4 identifies $\delta_{i}(\tilde{x})$ at those $\tilde{x}$ for which an open subset $\omega^{\prime} \subseteq \omega^{*}$ with $\operatorname{Pr}\left(X \in \omega^{\prime} \mid \tilde{x}\right)>0$ is known. In Appendix B, we present sufficient conditions for existence of such a $\omega^{\prime}$ that also satisfies the NDS condition. Essentially these conditions require the density of $\mathcal{S}_{i}$ given $\tilde{x}$ to be bounded away from zero over intervals between $\alpha_{i} x_{i}+\delta_{i}(\tilde{x})$ and $\alpha_{i} x_{i}$ for any $\left(x_{e}, \tilde{x}\right) \in \omega^{\prime}{ }^{12}$ The next subsection discusses how to test the assumption that a given set of states belongs to $\omega^{*}$.

\footnotetext{
${ }^{12}$ This condition is sufficient for the system in $(3)$ to admit only one solution for all $\left(x_{e}, \tilde{x}\right) \in \omega^{\prime}$ when $h_{i}\left(D_{-i}\right)=$ $\sum_{j \neq i} D_{j}$ and $u_{i}\left(x, \epsilon_{i}\right)$ is additively separable in $x$ and $\epsilon_{i}$ for both $i$. It is stronger than what we need because there can be a unique BNE in the DGP even when the system admits multiple solutions at a state $x$.
} 
Now consider extending the arguments that identify $\tilde{u}_{i}(\tilde{x})$ in Section 3 under the mean independence of $\epsilon_{i}$ to allow for multiple BNE in data. The idea is to use the variation of $X_{i}$ as before, but now only over some subset states in $\omega^{*}$.

$\mathbf{A 5}^{\prime \prime \prime}$ For any $i$ and $\tilde{x}$, there exists some $x_{-i}=\left(x_{e,-i}, \tilde{x}\right)$ and $\omega_{i} \subseteq \Omega_{X_{i} \mid x_{-i}}$ such that $(i) \omega_{i}$ is an interval with $\operatorname{Pr}\left(X_{i} \in \omega_{i} \mid x_{-i}\right)>0$ and $\omega_{i} \otimes x_{-i} \subseteq \omega^{*}$; (ii) the support of $V_{i}$ conditional on $X_{i} \in \omega_{i}$ and $x_{-i}$ includes the support of $\mathcal{S}_{i}$ given $\tilde{x}$; (iii) the density of $X_{i}$ conditional on $X_{i} \in \omega_{i}$ and $x_{-i}$ is positive a.e. on $\omega_{i}$; and (iv) the sign of $\partial V_{i}(X) /\left.\partial X_{i}\right|_{X=\left(x_{i}, x_{-i}\right)}$ is identical to the sign of $\alpha_{i}$ for all $x_{i} \in \omega_{i}$.

$\mathrm{A} 5^{\prime \prime \prime}$ is more restrictive than $\mathrm{A} 5$, because $\mathrm{A} 5^{\prime \prime \prime}$ requires the large support condition and the monotonicity condition to hold over a set of states with a unique equilibrium in DGP. The presence of excluded regressors with the assumed support properties in our model generally implies that $\omega^{*}$ is not empty. An intuitive sufficient condition for $\mathrm{A} 5^{\prime \prime \prime}$ to hold for some $i$ and $\tilde{x}$ is that the other excluded regressors (except $x_{i}$ ) take extremely large or small values so that the system (3) admits a unique solution. (To see this, consider the example of entry game. Suppose the observed component of fixed costs, or excluded regressors, for all but one firm take on extremely large values. Then the probability of entry for all but one of the firms are practically zero, yielding a unique BNE where the remaining one player with a non-extreme fixed cost component $X_{i}$ enters if and only if his monopoly profit exceeds his fixed cost of entry.)

Nonetheless, note that such extreme values are not necessary for $\omega^{*}$ to be non-empty and for $\mathrm{A} 5^{\prime \prime \prime}$ to hold. This is because the equilibrium selection mechanism may well be such that even for states with non-extreme values the data is rationalized by a single BNE. For instance, in the extreme case of $\omega^{*}=\Omega_{X}$ we would be back to the scenario as assumed in A2. Conditions (i) and (iii) in $\mathrm{A} 5^{\prime \prime \prime}$ are mild regularity conditions. Condition (iv) in $\mathrm{A} 5^{\prime \prime \prime}$ can be satisfied when $\delta_{i}(\tilde{x})$ and the density of $\epsilon_{i}$ given $\tilde{x}$ are bounded above. We formalize primitive conditions that are sufficient for the existence of such $x_{-i}$ and $\omega_{i}$ satisfying $\mathrm{A} 5^{\prime \prime \prime}$ in Appendix B.

For any $x_{-i}$ and $\omega_{i}$, define $Y_{i, H}^{\prime}$ by replacing $f\left(x_{i} \mid x_{-i}\right)$ with $f\left(x_{i} \mid x_{-i}, \omega_{i}\right)$ in the definition of $Y_{i, H}$ in (14). Choose a continuous distribution $H$ as in (14), but now require it to have an interval support contained in the support of $V_{i}$ conditional on " $X_{-i}=x_{-i}$ and $X_{i} \in \omega_{i}$ ". Let $\mu_{H}$ denote the expectation with respect to the chosen distribution $H$. Again, the potential dependence of $H$ (and $\left.\mu_{H}\right)$ on $\left(x_{-i}, \omega_{i}\right)$ is suppressed to simplify notations.

Corollary 5 (Theorem 3) Suppose A1,3,4 hold. For any $i, x_{-i}$ and $\omega_{i}$ such that A5'" holds,

$$
\tilde{u}_{i}(\tilde{x})=E\left[Y_{i, H}^{\prime} \mid X_{i} \in \omega_{i}, x_{-i}\right]-\mu_{H} .
$$

Once we condition on $x_{-i}$ and $\omega_{i}$ with $\omega_{i} \otimes x_{-i} \subseteq \omega^{*}$, the link between model primitives and 
distribution of states and actions observed from data takes the form of (1). The proof of Corollary 5 is then an extension of Corollary 2, and is omitted for brevity.

Similar to the case with unique BNE, we could also modify the identifying argument in Corollary 5 to exploit the variation of $X_{e}$ over a set of states with unique BNE and $\tilde{X}=\tilde{x}$. This would be done by replacing the denominator of (11) with $f_{V_{i}}\left(V_{i}(x) \mid X_{e} \in \omega_{e}, \tilde{x}\right)$, where $\omega_{e}$ consists of a set of $x_{e}$ such that: (a) $\operatorname{Pr}\left(X_{e} \in \omega_{e} \mid \tilde{x}\right)>0$; (b) $\omega_{e} \otimes \tilde{x} \subseteq \omega^{*}$; and (c) the support of $V_{i}$ given " $\tilde{X}=\tilde{x}$ and $X_{e} \in \omega_{e}$ " covers that of $-\tilde{u}_{i}(\tilde{x})+\epsilon_{i}$ given $\tilde{x}$.

\subsection{Testing the assumption of unique equilibrium in a subset of states}

When there may be multiple BNE in data under some states, estimation of $\alpha_{i}, \delta_{i}(\tilde{x})$ and $\tilde{u}_{i}(\tilde{x})$ requires knowledge of some subset of $\omega^{*}$. Specifically, the researcher needs to know some $\omega_{e} \subseteq \Omega_{X_{e} \mid \tilde{x}}$ with $\omega_{e} \otimes \tilde{x} \subseteq \omega^{*}$ in order to estimate $\alpha_{i}, \delta_{i}(\tilde{x})$, and some $\omega_{i} \subseteq \Omega_{X_{i} \mid x_{-i}}$ with $\omega_{i} \otimes x_{-i} \subseteq \omega^{*}$ in order to estimate $\tilde{u}_{i}(\tilde{x})$. The goal of this subsection is to discuss how to test the assumption that a given set of states the researcher chooses to use for estimation is in fact a subset of $\omega^{*}$. More specifically, given some $\bar{\omega} \subset \Omega_{X}$, we modify the method in De Paula and Tang (2011) to propose a test for the null that for almost all states in $\bar{\omega}$ the actions observed in data are rationalized by a single BNE. For brevity we focus on the semiparametric case with $\delta_{i}(\tilde{x})=\delta_{i}$ and $N=2$.

For any generic $\bar{\omega} \subset \Omega_{X}$ with $\operatorname{Pr}(X \in \bar{\omega})>0$, define:

$$
T(\bar{\omega}) \equiv E\left[1\{X \in \bar{\omega}\}\left(D_{1} D_{2}-p_{1}^{*}(X) p_{2}^{*}(X)\right)\right]
$$

The following lemma is a special case of Proposition 1 in De Paula and Tang (2011), which builds on results in Manski (1993) and Sweeting (2009).

Lemma 2 Suppose $\delta_{i}(\tilde{x})=\delta_{i} \neq 0$ for all $\tilde{x}$ and $i=1,2$. Under $A 1$,

$$
\operatorname{Pr}\left\{X \notin \omega^{*} \mid X \in \bar{\omega}\right\}>0 \text { if and only if } T(\bar{\omega}) \neq 0
$$

The intuition for (24) is that, if players' private information sets are independent conditional on $x$, then their actions must be uncorrelated if the data are rationalized by a single BNE. Any correlations between actions in data can only occur as players simultaneously move between strategies prescribed in different BNE. De Paula and Tang (2011) showed this result in a general setting where both the baseline payoffs $u_{i}\left(x, \epsilon_{i}\right)$ and the interaction effects $\delta_{i}(x)$ are unrestricted functions of states $x$. They also propose the use of a multiple testing procedure to infer the signs of strategic interaction effects in addition to the presence of multiple BNE. 
Given Lemma 2, for any $\bar{\omega}$ with $\operatorname{Pr}\{X \in \bar{\omega}\}>0$, the formulation of the null and alternative as

$$
H_{0}: \operatorname{Pr}\left\{X \in \omega^{*} \mid X \in \bar{\omega}\right\}=1 \text { vs. } H_{A}: \operatorname{Pr}\left\{X \in \omega^{*} \mid X \in \bar{\omega}\right\}<1
$$

is equivalent to the formulation

$$
H_{0}: T(\bar{\omega})=0 \quad \text { vs. } H_{A}: T(\bar{\omega}) \neq 0 .
$$

Exploiting this equivalence, we propose a test statistic based on the analog principle and derive its asymptotic distribution. To simplify exposition, we focus on the case where $\tilde{X}$ is discrete (recall that $X_{e}$ is continuous). The generalization to the case with continuous coordinates in $\tilde{X}$ is straightforward.

For a product kernel $K$ with support in $\mathbb{R}^{2}$ and bandwidth $\sigma$ (with $\sigma \rightarrow 0$ as $G \rightarrow+\infty$ ), define $K_{\sigma}(.) \equiv \sigma^{-2} K(. / \sigma)$. Let $D_{g} \equiv\left(D_{g, 0}, D_{g, 1}, D_{g, 2}\right)$ where $D_{g, 0} \equiv 1, X_{g} \equiv\left(X_{g, e}, \tilde{X}_{g}\right)$ and $X_{g, e} \equiv\left(X_{g, 1}, X_{g, 2}\right)$ denotes actions and states observed in independent games, each of which is indexed by $g$. Define the statistic

$$
\hat{T}(\bar{\omega}) \equiv \frac{1}{G} \sum_{g \leq G} 1\left\{x_{g} \in \bar{\omega}\right\}\left[d_{1, g} d_{2, g}-\hat{p}_{1}\left(x_{g}\right) \hat{p}_{2}\left(x_{g}\right)\right]
$$

with

$$
\hat{p}_{i}(x) \equiv \hat{\gamma}_{i}(x) / \hat{\gamma}_{0}(x) \text { and } \hat{\gamma}_{k}(x) \equiv \frac{1}{G} \sum_{g} d_{g, k} K_{\sigma}\left(x_{g, e}-x_{e}\right) 1\left\{\tilde{x}_{g}=\tilde{x}\right\}
$$

for $i=1,2$ and $k=0,1,2 \cdot{ }^{13}$ Define $\hat{\gamma} \equiv\left[\hat{\gamma}_{0}, \hat{\gamma}_{1}, \hat{\gamma}_{2}\right]^{\prime}$. We then have

$$
\hat{T}(\bar{\omega})=\frac{1}{G} \sum_{g} 1\left\{x_{g} \in \bar{\omega}\right\} m\left(z_{g} ; \hat{\gamma}\right)
$$

where $z \equiv\left(d_{1}, d_{2}, x\right)$, and $m(z ; \gamma) \equiv d_{1} d_{2}-\gamma_{0}^{-2}(x) \gamma_{1}(x) \gamma_{2}(x)$ for a generic vector $\gamma \equiv\left[\gamma_{0}, \gamma_{1}, \gamma_{2}\right]$. For notation simplicity, suppress the dependence of $w$ and $m$ on $\left(\tilde{x}, \omega_{e}\right)$ when there is no ambiguity. Let $\gamma^{*} \equiv\left[\gamma_{0}^{*}, \gamma_{1}^{*}, \gamma_{2}^{*}\right]^{\prime}$, where $\gamma_{0}^{*}$ denotes the true density of $X$ and $\gamma_{i}^{*} \equiv p_{i}^{*} \gamma_{0}^{*}$ for $i=1,2$ (with $p_{i}^{*}$ being choice probabilities directly identified from data). Let $F^{*}, f^{*}$ denote the true distribution and density of $z$ in the DGP.

The detailed conditions needed to obtain the asymptotic distribution of $\hat{T}(\bar{\omega})(T 1-4)$ are collected in the supplement of this article (Lewbel and Tang (2012)). These conditions include assumptions on smoothness and boundedness of relevant population moments (T1, T4), the properties of kernel functions (T2) and properties of the sequence of bandwidth (T3).

Theorem 6 Suppose assumptions T1-4 hold and $\operatorname{Pr}\{X \in \bar{\omega}\}>0$. Then

$$
\sqrt{G}\left(\hat{T}(\bar{\omega})-\mu_{\bar{\omega}}\right) \stackrel{d}{\longrightarrow} N\left(0, \Sigma_{\bar{\omega}}\right)
$$

\footnotetext{
${ }^{13}$ Alternatively, we could replace the indicator function $1\left\{\tilde{x}_{g}=\tilde{x}\right\}$ with smooth kernels while estimating $\gamma$. These joint product kernels will be defined over $R^{J}$ and satisfy $\int\left|\tilde{K}\left(t_{1}, t_{2}, \mathbf{0}\right)\right| d t_{1} d t_{2}<\infty ; \int \tilde{K}\left(t_{1}, t_{2}, \mathbf{0}\right) d t_{1} d t_{2}=1 ;$ and for any $\eta>0, \sqrt{G} \sup _{|| \tilde{t}||>\eta / \sigma} \int\left|\tilde{K}\left(t_{1}, t_{2}, \tilde{\mathbf{t}}\right)\right| d t_{1} d t_{2} \rightarrow 0$. See Bierens (1987) for details.
} 
where $\mu_{\bar{\omega}} \equiv E\left[1\{X \in \bar{\omega}\}\left(D_{1} D_{2}-p_{1}^{*}(X) p_{2}^{*}(X)\right)\right]$ and $\Sigma_{\bar{\omega}}$ is defined as

$$
\operatorname{Var}\left[1\{X \in \bar{\omega}\}\left(D_{1} D_{2}-p_{1}^{*}(X) p_{2}^{*}(X)+\frac{2 p_{1}^{*}(X) p_{2}^{*}(X)-D_{1} p_{2}^{*}(X)-D_{2} p_{1}^{*}(X)}{\gamma_{0}^{*}(X)}\right)\right] .
$$

The covariance matrix $\Sigma_{\bar{\omega}}$ above is expressed in terms of functions that are observable in the data, and so can be consistently estimated by its sample analog under standard conditions.

\section{Conclusions}

We have provided conditions for nonparametric identification of static binary games with incomplete information, and associated estimators that converge at parametric rates for a semiparametric specification of the model with linear payoffs. The point identification extends to allow for the presence of multiple Bayesian Nash equilibria in the data-generating process. We show that assumptions required for identification are plausible and have ready economic interpretations in contexts such as simultaneous market entry decisions by firms.

Our identification and estimation methods depend critically on observing excluded regressors, which are state variables that affect just one player's payoffs, and do so in an additively separable way. Like all public information affecting payoffs, excluded variables still affect all player's probabilities of actions. Nevertheless, we show that a function of excluded regressors and payoffs can be identified and constructed that play the role of special regressors for identifying the model. Full identification of the model requires relatively large variation in these excluded regressors, to give these constructed special regressors a large support.

In the entry game example, observed components of fixed costs are natural examples of excluded regressors. An obstacle to applying our results in practice is that data on components of fixed costs, while public, is often not collected, perhaps because existing empirical applications of entry games (which generally require far more stringent assumptions than our model) do not require observation of fixed cost components for identification. We hope our results will provide the incentive to collect such data in the future.

Our results may also be applicable to different types of games, e.g. they might be used to identify and estimate the outcomes of household bargaining models, given sufficient data on the components of each spouse's payoffs. 


\section{Appendix A: Proofs of Identification Results}

Proof of Theorem 1. For any $\tilde{x}$, let $\mathcal{D}(\tilde{x})$ denote a $N$-vector with ordered coordinates $\left(\delta_{1}(\tilde{x}), \delta_{2}(\tilde{x})\right.$, .., $\left.\delta_{N}(\tilde{x})\right)$. For any $x \equiv\left(x_{e}, \tilde{x}\right)$, let $\mathcal{F}(x)$ denote a $N$-vector with the $i$-th coordinate being $f_{\mathcal{S}_{i} \mid \tilde{x}}\left(\alpha_{i} x_{i}+\delta_{i}(\tilde{x}) \phi_{i}^{*}(x)\right)$. Under Assumptions 1-3, for any $x$ and all $i$,

$$
p_{i}^{*}(x)=\operatorname{Pr}\left\{\mathcal{S}_{i} \leq \alpha_{i} X_{i}+\delta_{i}(\tilde{X}) \phi_{i}^{*}(X) \mid X=x\right\} .
$$

Now for any $x$, fix $\tilde{x}$ and differentiate both sides of (26) with respect to $X_{i}$ for all $i$. This gives:

$$
\mathcal{W}_{1}(x)=\mathcal{F}(x) \cdot\left(\mathcal{A}+\mathcal{D}(\tilde{x}) \cdot \mathcal{V}_{1}(x)\right)
$$

where "." denotes component-wise multiplication. Furthermore, for $i \leq N-1$, differentiate both sides of (26) w.r.t. $X_{i+1}$ at $x$. Also differentiate both sides of (26) for $i=N$ with respect to $X_{1}$ at $x$. Stacking $N$ equations resulting from these differentiations, we have

$$
\mathcal{W}_{2}(x)=\mathcal{F}(x) \cdot \mathcal{D}(\tilde{x}) \cdot \mathcal{V}_{2}(x)
$$

For all $x \in \omega$ (where $\omega$ satisfies NDS), none of the coordinates in $\mathcal{F}(x), \mathcal{V}_{2}(x)$ and $\mathcal{V}_{2}(x) . \mathcal{W}_{1}(x)-$ $\mathcal{V}_{1}(x) . \mathcal{W}_{2}(x)$ are zero. It then follows that for any $x \in \omega$,

$$
\begin{aligned}
& \mathcal{A} . \mathcal{F}(x)=\mathcal{W}_{1}(x)-\mathcal{W}_{2}(x) . \mathcal{V}_{1}(x) . / \mathcal{V}_{2}(x) \\
& \mathcal{D}(\tilde{x})=\mathcal{A} . \mathcal{W}_{2}(x) . /\left[\mathcal{V}_{2}(x) . \mathcal{W}_{1}(x)-\mathcal{V}_{1}(x) . \mathcal{W}_{2}(x)\right]
\end{aligned}
$$

where $\mathcal{A}$ is a constant vector and "./" denotes component-wise divisions. Note $\mathcal{F}(x)$ is a vector with strictly positive coordinates under Assumption 1. Hence signs of $\alpha_{i}$ 's must be identical to those of coordinates on the right-hand side in (29) for any $x \in \omega$. Integrating out $X$ over $\omega$ using the marginal distribution of $X$ gives (7). The equation (30) suggests $\mathcal{D}_{i}(\tilde{x})$ is over-identified from the right-hand side for all $x \in \omega$. Integrating out $X$ over $\omega$ using the distribution of $X$ conditional on $\tilde{x}$ and $X \in \omega$ gives (8). Q.E.D.

Proof of Corollary 2. Fix a $x_{-i}=\left(x_{e,-i}, \tilde{x}\right)$ that satisfies A5'. Then

$$
\begin{aligned}
& E\left[Y_{i, H} \mid x_{-i}\right]=\int \frac{\left[E\left(D_{i} \mid X=x\right)-H\left(V_{i}(x)\right)\right]\left[1+\alpha_{i} \delta_{i}(\tilde{x}) \phi_{i, i}^{*}(x)\right]}{f_{X_{i}}\left(x_{i} \mid x_{-i}\right)} f_{X_{i}}\left(x_{i} \mid x_{-i}\right) d x_{i} \\
= & \int\left[E\left(D_{i} \mid V_{i}=V_{i}(x), \tilde{x}\right)-H\left(V_{i}(x)\right)\right]\left[1+\alpha_{i} \delta_{i}(\tilde{x}) \phi_{i, i}^{*}(x)\right] d x_{i}
\end{aligned}
$$

where the first equality follows from the law of iterated expectations and A5'-(ii); and the second from A3 and that $\epsilon_{i} \perp V_{i}(X)$ given $\tilde{x}$. The integrand in (31) is continuous in $V_{i}(X)$, and $V_{i}(X)$ is continuously differentiable in $X_{i}$ given $x_{-i}$ under A1 and A3, with $\left.\frac{\partial V_{i}(X)}{\partial X_{i}}\right|_{X=x}=\alpha_{i}+\delta_{i}(\tilde{x}) \phi_{i, i}^{*}(x)$. We suppress the dependence of $\bar{v}_{i}, \underline{v}_{i}, \mu_{H}$ on $x_{-i}$ to simplify notations in the proof. Consider the case 
with $\alpha_{i}=1$ so that $V_{i}$ is increasing in $x_{i}$ given $x_{-i}$ under A5'-(iii). In this case, $1+\alpha_{i} \delta_{i}(\tilde{x}) \phi_{i, i}^{*}(x)=$ $\alpha_{i}+\delta_{i}(\tilde{x}) \phi_{i, i}^{*}(x)$, and a change of variables between $v_{i}$ and $x_{i}$ while holding $x_{-i}$ fixed gives us

$$
E\left[Y_{i, H} \mid x_{-i}\right]=\int_{\underline{v}_{i}}^{\bar{v}_{i}}\left[E\left(D_{i} \mid v, \tilde{x}\right)-H(v)\right] d v=\int_{\underline{v}_{i}}^{\bar{v}_{i}}\left[E\left(D_{i} \mid v, \tilde{x}\right)-1\left\{v \geq \mu_{H}\right\}\right] d v,
$$

where the second equality uses integration by parts and the properties of $H$. It also uses that $\mu_{H}$ is in the interior of support of $H$. Then

$$
\begin{aligned}
& E\left[Y_{i, H} \mid x_{-i}\right]=\int_{\underline{v}_{i}}^{\bar{v}_{i}}\left(\int_{\Omega_{\epsilon_{i} \mid \tilde{x}}} 1\left\{\varepsilon_{i} \leq \tilde{u}_{i}(\tilde{x})+v\right\} d F\left(\varepsilon_{i} \mid \tilde{x}\right)\right)-1\left\{v \geq \mu_{H}\right\} d v \\
= & \int_{\Omega_{\epsilon_{i} \mid \tilde{x}}} \int_{\underline{v}_{i}}^{\bar{v}_{i}} 1\left\{v \geq s_{i}\right\}-1\left\{v \geq \mu_{H}\right\} d v d F\left(\varepsilon_{i} \mid \tilde{x}\right),
\end{aligned}
$$

where $s_{i} \equiv-\tilde{u}_{i}(\tilde{x})+\varepsilon_{i}$ and the last equality follows from a change of the order of integration between $v$ and $\varepsilon_{i}$ allowed under the support condition in A5'-(i) and the Fubini's Theorem. Then $E\left[Y_{i, H} \mid x_{-i}\right]$ becomes

$$
\begin{aligned}
& \int_{\Omega_{\epsilon_{i} \mid \tilde{x}}} \int_{\underline{v}_{i}}^{\bar{v}_{i}} 1\left\{s_{i} \leq v<\mu_{H}\right\} 1\left\{s_{i} \leq \mu_{H}\right\}-1\left\{\mu_{H}<v \leq s_{i}\right\} 1\left\{s_{i}>\mu_{H}\right\} d v d F\left(\epsilon_{i} \mid \tilde{x}\right) \\
= & \int_{\Omega_{\epsilon_{i} \mid \tilde{x}}}\left(1\left\{s_{i} \leq \mu_{H}\right\} \int_{s_{i}}^{\mu_{H}} d v-1\left\{s_{i}>\mu_{H}\right\} \int_{\mu_{H}}^{s_{i}} d v\right) d F\left(\epsilon_{i} \mid \tilde{x}\right) \\
= & \int_{\Omega_{\epsilon_{i} \mid \tilde{x}}}\left(\mu_{H}-s_{i}\right) d F\left(\epsilon_{i} \mid \tilde{x}\right)=\tilde{u}_{i}(\tilde{x})+\mu_{H} .
\end{aligned}
$$

Next consider the case with $\alpha_{i}=-1$ so that $V_{i}$ is decreasing in $x_{i}$ given $x_{-i}$ under A5'-(iii). By construction $1+\alpha_{i} \delta_{i}(\tilde{x}) \phi_{i, i}^{*}(x)=-\alpha_{i}-\delta_{i}(\tilde{x}) \phi_{i, i}^{*}(x)=-\left.\frac{\partial V_{i}(X)}{\partial X_{i}}\right|_{X=x}$. The proof then follows from change of variables as above. Q.E.D.

Proof of Lemma 2. Under A1,

$$
x \in \omega^{*} \text { if and only if } E\left[D_{1} D_{2} \mid X=x\right]=p_{1}^{*}(x) p_{2}^{*}(x),
$$

and $\operatorname{sign}\left(E\left[D_{1} D_{2} \mid X=x\right]-p_{1}^{*}(x) p_{2}^{*}(x)\right)=\operatorname{sign}\left(\delta_{i}\right)$ for all $x \notin \omega^{*}$. (See Proposition 1 in De Paula and Tang (2011) for details.)

First suppose $\operatorname{Pr}\left\{X \in \omega^{*} \mid X \in \bar{\omega}\right\}=1$. Integrating out $X$ in (32) given " $X \in \bar{\omega}$ " shows $E\left[D_{1} D_{2}-p_{1}^{*}(X) p_{2}^{*}(X) \mid X \in \bar{\omega}\right]$, which in turn implies $T(\bar{\omega})=0$. Next suppose $\operatorname{Pr}\left\{X \notin \omega^{*} \mid X \in\right.$ $\bar{\omega}\}>0$. Then $\operatorname{sign}\left(\delta_{1}\right)$ must necessarily equal $\operatorname{sign}\left(\delta_{2}\right)$. (Otherwise, there can't be multiple BNE at any state $x$.) The set of states in $\bar{\omega}$ can be partitioned into its intersection with $\omega^{*}$ and its intersection with the complement of $\omega^{*}$ in the support of states. For all $x$ in the latter intersection, $E\left[D_{1} D_{2} \mid x\right]-p_{1}^{*}(x) p_{2}^{*}(x)$ is non-zero and its sign is equal to $\operatorname{sign}\left(\delta_{i}\right)$ for $i=1,2$. For all $x$ in the 
former intersection (with $\left.\omega^{*}\right), E\left[D_{1} D_{2} \mid x\right]-p_{1}^{*}(x) p_{2}^{*}(x)$ is equal to 0. Applying the Law of Iterated Expectations using these two partitioning intersections shows $E\left[D_{1} D_{2}-p_{1}^{*}(X) p_{2}^{*}(X) \mid X \in \bar{\omega}\right] \neq 0$ and its sign is equal to $\operatorname{sign}\left(\delta_{i}\right)$. This in turn implies $T(\bar{\omega}) \neq 0$ with $\operatorname{sign}(T(\bar{\omega}))=\operatorname{sign}\left(\delta_{i}\right)$ for $i=1,2$. Q.E.D.

\section{Appendix B: NDS, Large Support and Monotonicity Conditions}

In this section, we provide conditions on the model primitives that are sufficient for some of the main identifying restrictions stated in the text. These include the NDS condition for identifying $\alpha_{i}$ and $\delta_{i}(\tilde{x})$; the large support condition in $\mathrm{A} 5, \mathrm{~A} 5{ }^{\prime}, \mathrm{A} 5{ }^{\prime \prime}$ and $\mathrm{A} 5^{\prime \prime \prime}$ for identifying component of the baseline payoff $\tilde{u}_{i}(\tilde{x}) ;$, as well as the monotonicity condition in A5', A5" and A5"' that the sign of marginal effects of excluded regressors on generated special regressors are identical to the sign of $\alpha_{i}$.

\section{B1. Sufficient conditions for NDS}

We now present fairly mild sufficient conditions for there to exist a subset of states $\omega \subseteq \omega^{*}$ that satisfies NDS. Consider a game with $N=2$ (with players denoted by 1 and 2) and $h_{i}\left(D_{j}\right)=D_{j}$ for $i=1,2$ and $j=3-i$. We begin by reviewing the sufficient conditions under which the system characterizing BNE in (3) admits only a unique solution at $x$. Let $p \equiv\left(p_{1}, p_{2}\right)$ and define:

$$
\left.\varphi(p ; X) \equiv\left[\begin{array}{c}
\varphi_{1}(p ; X) \\
\varphi_{2}(p ; X)
\end{array}\right] \equiv\left[\begin{array}{c}
p_{1}-F_{\mathcal{S}_{1} \mid \tilde{X}}\left(\alpha_{1} X_{1}+\delta_{1}(\tilde{X}) p_{2}\right) \\
p_{2}-F_{\mathcal{S}_{2} \mid \tilde{X}}\left(\alpha_{2} X_{2}+\delta_{2}(\tilde{X}) p_{1}\right.
\end{array}\right)\right]
$$

By Theorem 7 in Gale and Nikaido (1965) and Proposition 1 in Aradillas-Lopez (2010), the solution for $p$ in the fixed point equation $p=\varphi(p ; x)$ at any $x$ is unique if none of the principal minors of the Jacobian of $\varphi(p ; x)$ with respect to $p$ vanishes on $[0,1]^{2}$. Or equivalently, if $\Pi_{i=1,2} \delta_{i}(\tilde{x}) f_{\mathcal{S}_{i} \mid \tilde{x}}\left(\alpha_{i} x_{i}+\right.$ $\left.\delta_{i}(\tilde{x}) p_{3-i}(x)\right) \neq 1$ for all $p \in[0,1]^{2}$ at $x$. Let $I_{i}(\tilde{x})$ denote the interval between 0 and $\delta_{i}(\tilde{x})$ for any $\tilde{x}$. That is, $I_{i}(\tilde{x}) \equiv\left[0, \delta_{i}(\tilde{x})\right]$ if $\delta_{i}(\tilde{x})>0$ and $\left[\delta_{i}(\tilde{x}), 0\right]$ otherwise. For any $\tilde{x}$, define:

$$
\begin{aligned}
\omega^{a}(\tilde{x}) & \equiv\left\{x_{e}: f_{\mathcal{S}_{i} \mid \tilde{x}}\left(t+\alpha_{i} x_{i}\right) \text { bounded away from } 0 \text { for all } t \in I_{i}(\tilde{x}) \text { and } i=1,2\right\} ; \text { and } \\
\omega^{b}(\tilde{x}) & \equiv\left\{x_{e}: \begin{array}{l}
\prod_{i} \min _{t \in I_{i}(\tilde{x})} f_{\mathcal{S}_{i} \mid \tilde{x}}\left(t+\alpha_{i} x_{i}\right)>\left(\delta_{1}(\tilde{x}) \delta_{2}(\tilde{x})\right)^{-1} \\
\prod_{i} \max _{t \in I_{i}(\tilde{x})} f_{\mathcal{S}_{i} \mid \tilde{x}}\left(t+\alpha_{i} x_{i}\right)<\left(\delta_{1}(\tilde{x}) \delta_{2}(\tilde{x})\right)^{-1}
\end{array}\right\}
\end{aligned}
$$

Proposition B1 Suppose A1,3 hold with $N=2$. (a) If $\delta_{1}(\tilde{x}) \delta_{2}(\tilde{x})<0$ and $\operatorname{Pr}\left\{X_{e} \in \omega^{a}(\tilde{x}) \mid \tilde{x}\right\}>0$, then $\omega^{a}(\tilde{x}) \otimes \tilde{x} \subseteq \omega^{*}$ and satisfies NDS. (b) If $\delta_{1}(\tilde{x}) \delta_{2}(\tilde{x})>0$ and $\operatorname{Pr}\left\{X_{e} \in \omega^{a}(\tilde{x}) \cap \omega^{b}(\tilde{x}) \mid \tilde{x}\right\}>0$, then $\left(\omega^{a}(\tilde{x}) \cap \omega^{b}(\tilde{x})\right) \otimes \tilde{x} \subseteq \omega^{*}$ and satisfies NDS. 
Proof of Proposition B1. First consider $\tilde{x}$ with $\Pi_{i=1,2} \delta_{i}(\tilde{x})<0$. Then $\Pi_{i=1,2} \delta_{i}(\tilde{x}) f_{\mathcal{S}_{i} \mid \tilde{x}}\left(\alpha_{i} x_{i}+\right.$ $\left.\delta_{i}(\tilde{x}) p_{3-i}(x)\right)<0$ for all $x_{e} \in \omega^{a}(\tilde{x})$ and all $p$ on $[0,1]^{2}$. It then follows that $\left(x_{e}, \tilde{x}\right) \in \omega^{*}$ for all $x_{e} \in \omega^{a}(\tilde{x})$. That $\left.\frac{\partial p_{i}^{*}(X)}{\partial X_{i}}\right|_{X=x},\left.\frac{\partial p_{i}^{*}(X)}{\partial X_{3-i}}\right|_{X=x}$ exist for $i=1,2$ a.e. on $\omega^{a}(\tilde{x})$ follows from the exclusion restrictions and smoothness conditions in Assumption 3. We now show $\operatorname{Pr}\left\{p_{i, i}^{*}(X) p_{j, j}^{*}(X)\right.$ $\neq p_{i, j}^{*}(X) p_{j, i}^{*}(X)$ and $\left.p_{j, j}^{*}(X) \neq 0 \mid X_{e} \in \omega^{a}(\tilde{x}), \tilde{X}=\tilde{x}\right\}=1$. Suppose this probability is strictly less than 1 for $i=1$. Suppose $\operatorname{Pr}\left\{\frac{\partial p_{2}^{*}(X)}{\partial X_{2}}=0 \mid X_{e} \in \omega^{a}(\tilde{x}), \tilde{X}=\tilde{x}\right\}>0$. By (5)-(6) for $i=1$, this implies there is a set of $\left(x_{1}, x_{2}\right)$ on $\omega^{a}(\tilde{x})$ with positive probability such that both $\frac{\partial p_{2}^{*}(x)}{\partial X_{2}}$ and $\frac{\partial p_{1}^{*}(x)}{\partial X_{2}}$ are 0 . But then by (5)-(6) for $i=2$ implies the same set of $\left(x_{1}, x_{2}\right)$ must satisfy $\frac{\partial p_{1}^{*}\left(x_{1}, x_{2}, \tilde{x}\right)}{\partial X_{2}}=-\alpha_{2} / \delta_{1}(\tilde{x}) \neq 0$, because the conditional densities of $\mathcal{S}_{i}$ over the relevant section of its domain is nonzero by definition of $\omega^{a}(\tilde{x})$. Contradiction. Hence $\operatorname{Pr}\left\{\frac{\partial p_{2}^{*}(X)}{\partial X_{2}}=0 \mid X_{e} \in \omega^{a}(\tilde{x})\right.$, $\tilde{X}=\tilde{x}\}=0$. Symmetric arguments prove the same statement with $\frac{\partial p_{2}^{*}(X)}{\partial X_{2}}$ replaced by $\frac{\partial p_{1}^{*}(X)}{\partial X_{1}}$. Now suppose $\operatorname{Pr}\left\{\frac{\partial p_{i}^{*}(X)}{\partial X_{i}} \frac{\partial p_{3-i}^{*}(X)}{\partial X_{3-i}}=\frac{\partial p_{i}^{*}(X)}{\partial X_{3-i}} \frac{\partial p_{3-i}^{*}(X)}{\partial X_{i}} \mid X_{e} \in \omega^{a}(\tilde{x}), \tilde{X}=\tilde{x}\right\}>0$. By construction, for all $x_{e}$ in $\omega^{a}(\tilde{x})$, the relevant conditional densities of $\mathcal{S}_{i}$ must be positive for $i=1,2$. Hence

$$
\frac{\partial p_{1}^{*}(x)}{\partial X_{1}} \frac{\partial p_{2}^{*}(x)}{\partial X_{2}}-\frac{\partial p_{1}^{*}(x)}{\partial X_{2}} \frac{\partial p_{2}^{*}(x)}{\partial X_{1}}=\frac{\alpha_{1}}{\delta_{1}(\tilde{x})} \frac{\partial p_{1}^{*}(x)}{\partial X_{2}}
$$

for all $x_{e}$ on $\omega^{a}(\tilde{x})$. Because $\operatorname{Pr}\left\{\frac{\partial p_{2}^{*}(X)}{\partial X_{2}} \neq 0 \mid X_{e} \in \omega^{a}(\tilde{x}), \tilde{X}=\tilde{x}\right\}=1$ as argued above, (5)(6) for $i=1$ and the definition of $\omega^{a}(\tilde{x})$ implies $\operatorname{Pr}\left\{\frac{\partial p_{1}^{*}(X)}{\partial X_{2}} \neq 0 \mid X_{e} \in \omega^{a}(\tilde{x}), \tilde{X}=\tilde{x}\right\}=1$. Hence $\operatorname{Pr}\left\{p_{1,1}^{*}(X) p_{2,2}^{*}(X)=p_{1,2}^{*}(X) p_{2,1}^{*}(X) \mid X_{e} \in \omega^{a}(\tilde{x}), \tilde{X}=\tilde{x}\right\}=0$. This proves part (a). Next consider $\tilde{x}$ with $\delta_{1}(\tilde{x}) \delta_{2}(\tilde{x})>0$. Because $\omega^{a}(\tilde{x}) \cap \omega^{b}(\tilde{x}) \subseteq \omega^{a}(\tilde{x})$, arguments in the proof of part (a) applies to show that the non-zero requirements in the NDS condition must hold over $\left(\omega^{a}(\tilde{x}) \cap \omega^{b}(\tilde{x})\right) \otimes \tilde{x}$ under Assumptions 1,3. Furthermore, by construction, for any $\left(x_{1}, x_{2}\right)$ in $\omega^{b}(\tilde{x})$, either $\Pi_{i} \delta_{i}(\tilde{x}) f_{\mathcal{S}_{i} \mid \tilde{x}}\left(\alpha_{i} x_{i}+\delta_{i}(\tilde{x}) p_{3-i}(x)\right)>1$ or $\Pi_{i} \delta_{i}(\tilde{x}) f_{\mathcal{S}_{i} \mid \tilde{x}}\left(\alpha_{i} x_{i}+\delta_{i}(\tilde{x}) p_{3-i}(x)\right)<1$ for all $p \in[0,1]^{2}$. Hence the BNE (as solutions to (3)) must be unique for all states in $\omega^{b}(\tilde{x}) \otimes \tilde{x}$. This proves $\left(\omega^{a}(\tilde{x}) \cap \omega^{b}(\tilde{x})\right) \otimes \tilde{x}$. Q.E.D.

\section{B2. Sufficient conditions for various large support conditions}

We begin with sufficient conditions on model primitives that are sufficient for the large support condition in $\mathrm{A} 55^{\prime \prime \prime}$. We will then give primitive conditions that are sufficient for large support restrictions in A5' and A5". Consider the case with $N=2$. For any $\tilde{x}$, let $s_{i l}, s_{i h}$ denote the infimum and supremum of the conditional support of $\mathcal{S}_{i}$ given $\tilde{x}$ respectively, and let $\delta_{i}^{+}(\tilde{x}) \equiv$ $\max \left\{0, \delta_{i}(\tilde{x})\right\}$ and $\delta_{i}^{-}(\tilde{x}) \equiv \min \left\{0, \delta_{i}(\tilde{x})\right\}$.

Proposition B2 Let $N=2$ and $h_{i}\left(D_{j}\right)=D_{j}$ for both $i=1,2$ and $j=3-i$, and let A1,3 hold. Suppose for $i$ and $x_{-i}=\left(x_{e,-i}, \tilde{x}\right)$, there exists an interval $\omega_{i} \subseteq \Omega_{X_{i} \mid x_{-i}}$ such that $\omega_{i} \otimes x_{-i} \in \omega^{*}$ and the support of $\alpha_{i} X_{i}$ given $X_{i} \in \omega_{i}$ and $x_{-i}$ is an interval that contains $\left[s_{i l}-\delta_{i}^{+}(\tilde{x}), s_{i h}-\delta_{i}^{-}(\tilde{x})\right]$. Then the set $\omega_{i}$ satisfies (i) $\omega_{i}$ is an interval with $\operatorname{Pr}\left(X_{i} \in \omega_{i} \mid x_{-i}\right)>0$; and (ii) support of $S_{i}$ given $\tilde{x}$ is included in the support of $V_{i}$ given $x_{-i}$. 
Proof of Proposition B2. Because $\omega_{i} \otimes x_{-i} \in \omega^{*}$, the smoothness conditions in A1,3 imply for all $i$ that $p_{i}^{*}(x)$ is continuous in $x_{i}$ over $\omega_{i}$. Since $\omega_{i}$ is an interval (path-wise connected), the support of $V_{i}$ given $x_{-i}$ and $X_{i} \in \omega_{i}\left(x_{-i}\right)$ must also be an interval. By A2 and the condition in Proposition $\mathrm{C} 2, \operatorname{Pr}\left\{\alpha_{i} X_{i}+\delta_{i}^{-}(\tilde{X})>s_{i h} \mid x_{-i}, X_{i} \in \omega_{i}\right\}>0$. This implies $\operatorname{Pr}\left\{V_{i}>s_{i h} \mid x_{-i}, X_{i} \in \omega_{i}\right\}>0$. Symmetrically, $\operatorname{Pr}\left\{\alpha_{i} X_{i}+\delta_{i}^{+}(\tilde{X})<s_{i l} \mid x_{-i}, X_{i} \in \omega_{i}\right\}>0$ implies $\operatorname{Pr}\left\{V_{i}<s_{i l} \mid x_{-i}, X_{i} \in \omega_{i}\right\}>$ 0 . Since the support of $V_{i}$ conditional on $x_{-i}$ and $X_{i} \in \omega_{i}$ is an interval, the definition of $s_{i l}, s_{i h}$ suggests the conditional support of $V_{i}$ must contain the support of $\mathcal{S}_{i} \equiv-u_{i}(\tilde{X})+\epsilon_{i}$ given $\tilde{x}$. Q.E.D.

The large support condition in $\mathrm{A} 5^{\prime \prime \prime}$ holds if for all $\tilde{x}$ there exists $x_{-i}=\left(x_{e,-i}, \tilde{x}\right)$ that satisfies conditions in Proposition B2.

Next, we give sufficient primitive conditions for large support restrictions in A5' and A5". Under $\mathrm{A} 2, \omega^{*}=\Omega_{X}$. Hence with A2 in the background (as is the case with Sections 5 and 6 ), the large support condition in A5" holds when conditions in Proposition B2 are satisfied by $\omega_{i}=\Omega_{X_{i} \mid x_{-i}}$ for all $x_{-i} \in \Omega_{X_{-i} \mid \tilde{x}}$. Furthermore, under A2, the large support condition in A5' is satisfied as long as the conditions in Proposition B2 are satisfied by $\omega_{i}=\Omega_{X_{i} \mid x_{-i}}$ by some $x_{-i} \in \Omega_{X_{-i} \mid \tilde{x}}$. The large support condition in A5 is implied by that in A5'. As stated in the text, these various versions of large support conditions are verifiable using observed distributions.

\section{B3. Sufficient conditions for monotonicity conditions}

For simplicity in exposition, consider the case with $N=2, h\left(D_{-i}\right)=D_{j}$ and $\alpha_{i}=-1$. For all $x \in \Omega_{X}$, we can solve for $p_{j, i}(x)$ using the system of fixed point equation in the marginal effects of excluded regressors on the choice probabilities. By construction, this gives:

$$
\partial V_{i}(X) /\left.\partial X_{i}\right|_{X=\left(x_{e}, \tilde{x}\right)}=-1+\delta_{i}(\tilde{x}) p_{j, i}(x)=\frac{1}{\delta_{i}(\tilde{x}) \delta_{j}(\tilde{x}) \tilde{f}_{i}(x) \tilde{f}_{j}(x)-1}
$$

as $\delta_{i}(\tilde{x}) \delta_{j}(\tilde{x}) \tilde{f}_{i}(x) \tilde{f}_{j}(x) \neq 1$ almost surely. Thus the sign of $\partial V_{i}(X) /\left.\partial X_{i}\right|_{X=\left(x_{e}, \tilde{x}\right)}$ is identical to the sign of $\delta_{i}(\tilde{x}) \delta_{j}(\tilde{x}) \tilde{f}_{i}(x) \tilde{f}_{j}(x)-1$. For the monotonicity condition in A5" to hold over $x \in \Omega_{X}$, it suffices to have two positive constants $c_{1}, c_{2}$ such that $c_{1} c_{2}<1$ and $\operatorname{sign}\left(\delta_{i}(\tilde{x})\right)=\operatorname{sign}\left(\delta_{j}(\tilde{x})\right)$ and $\left|\delta_{i}(\tilde{x})\right|,\left|\delta_{j}(\tilde{x})\right| \leq c_{1}$ and $f_{\epsilon_{i} \mid \tilde{x}}, f_{\epsilon_{j} \mid \tilde{x}}$ bounded above by some positive constant $c_{2}$ uniformly over $\Omega_{\epsilon_{i} \mid \tilde{x}}, \Omega_{\epsilon_{i} \mid \tilde{x}}$ and $\tilde{x} \in \Omega_{\tilde{X}}$. This would imply the monotonicity condition in A5' as well.

In A5', the monotonicity condition is only required to hold for some $x_{-i}=\left(x_{e,-i}, \tilde{x}\right)$. Hence it can also hold without such uniform bound conditions in the previous paragraph. It suffices to have $\delta_{i}, \delta_{j}$ bounded and some $x_{e,-i}$ large or small enough so that the first term in the denominator is smaller than 1 for the $\tilde{x}$ and $x_{-i}=\left(x_{e,-i}, \tilde{x}\right)$ considered. 


\section{References}

[1] Aguirregabiria, V. and P. Mira, "A Hybrid Genetic Algorithm for the Maximum Likelihood Estimation of Models with Multiple Equilibria: A First Report," New Mathematics and Natural Computation, 1(2), July 2005, pp: 295-303.

[2] Aradillas-Lopez, A., "Semiparametric Estimation of a Simultaneous Game with Incomplete Information", Journal of Econometrics, Volume 157 (2), Aug 2010, pp: 409-431

[3] Andrews, D.K. and M. Schafgans, "Semiparametric Estimation of the Intercept of a Sample Selection Model," Review of Economic Studies, Vol. 65, 1998, pp:497-517

[4] Bajari, P., J. Hahn, H. Hong and G. Ridder, "A Note on Semiparametric Estimation of Finite Mixtures of Discrete Choice Models with Application to Game Theoretic Models"' forthcoming, International Economic Review

[5] Bajari, P., Hong, H., Krainer, J. and D. Nekipelov, "Estimating Static Models of Strategic Interactions," Journal of Business and Economic Statistics 28(4), 469-482 (2010)

[6] Bajari, P., Hong, H. and S. Ryan, "Identification and Estimation of a Discrete Game of Compete Information," Econometrica, Vol. 78, No. 5 (September, 2010), 1529-1568.

[7] Beresteanu, A., Molchanov, I. and F. Molinari, "Sharp Identification Regions in Models with Convex Moment Predictions," Econometrica, forthcoming, 2011

[8] Berry, S. and P. Haile, "Identification in Differentiated Products Markets Using Market Level Data", Cowles Foundation Discussion Paper No.1744, Yale University, 2010

[9] Berry, S. and P. Haile, "Nonparametric Identification of Multinomial Choice Demand Models with Heterogeneous Consumers", Cowles Foundation Discussion Paper No.1718, Yale University, 2009

[10] Blume, L., Brock, W., Durlauf, S. and Y. Ioannides, "Identification of Social Interactions," Handbook of Social Economics, J. Benhabib, A. Bisin, and M. Jackson, eds., Amsterdam: North Holland, 2010.

[11] Bierens, H., "Kernel Estimators of Regression Functions", in: Truman F.Bewley (ed.), Advances in Econometrics: Fifth World Congress, Vol.I, New York: Cambridge University Press, 1987, 99-144.

[12] Brock, W., and S. Durlauf (2007): "Identification of Binary Choice Models with Social Interactions," Journal of Econometrics, 140(1), pp 52-75.

[13] Ciliberto, F., and E. Tamer, "Market Structure and Multiple Equilibria in Airline Markets," Econometrica, 77, 1791-1828. 
[14] De Paula, A. and X. Tang, "Inference of Signs of Interaction Effects in Simultaneous Games with Incomplete Information," working paper, University of Pennsylvania, 2011.

[15] Khan, S. and E. Tamer, "Irregular Identification, Support Conditions and Inverse Weight Estimation," forthcoming, Econometrica, June 2010.

[16] Klein, R., and W. Spady (1993): "An Efficient Semiparametric Estimator of Binary Response Models," Econometrica, 61, 387-421.

[17] Magnac, T. and E. Maurin, "Identification and Information in Monotone Binary Models," Journal of Econometrics, Vol 139, 2007, pp 76-104

[18] Magnac, T. and E. Maurin, "Partial Identification in Monotone Binary Models: Discrete Regressors and Interval Data," Review of Economic Studies, Vol 75, 2008, pp 835-864

[19] Lewbel, A. "Semiparametric Latent Variable Model Estimation with Endogenous or Mismeasured Regressors," Econometrica, Vol.66, Janurary 1998, pp.105-121

[20] Lewbel, A. "Semiparametric Qualitative Response Model Estimation with Unknown Heteroskaedasticity. or Instrumental Variables," Journal of Econometrics, 2000

[21] Lewbel, A. "An Overview of the Special Regressor Method," Boston College Working Paper, 2012.

[22] Lewbel, A., O. Linton, and D. McFadden, "Estimating Features of a Distribution From Binomial Data," Journal of Econometrics, 2011, 162, 170-188

[23] Lewbel, A. and X. Tang, "Supplement for 'Identification and Estimation of Games with Incomplete Information Using Excluded Regressors'", unpublished manuscript, 2012.

[24] Manski, C., "Identification of Endogenous Social Effects: The Selection Problem," Review of Economic Studies, 60(3), 1993, pp 531-542.

[25] Newey, W. and D. McFadden, "Large Sample Estimation and Hypothesis Testing,", Handbook of Econometrics, Vol 4, ed. R.F.Engle and D.L.McFadden, Elsevier Science, 1994

[26] Seim, K., "An Empirical Model of Firm Entry with Endogenous Product-Type Choices," The RAND Journal of Economics, 37(3), 2006, pp 619-640.

[27] Sweeting, A., "The Strategic Timing of Radio Commercials: An Empirical Analysis Using Multiple Equilibria," RAND Journal of Economics, 40(4), 710-742, 2009

[28] Tamer, E., "Incomplete Simultaneous Discrete Response Model with Multiple Equilibria," Review of Economic Studies, Vol 70, No 1, 2003, pp. 147-167. 
[29] Tang, X., "Estimating Simultaneous Games with Incomplete Information under Median Restrictions," Economics Letters, 108(3), 2010

[30] Wan, Y., and H. Xu, "Semiparametric Estimation of Binary Decision Games of Incomplete Information with Correlated Private Signals," Working Paper, Pennsylvania State University, 2010

[31] Xu, H., "Estimation of Discrete Game with Correlated Private Signals," Working Paper, Penn State University, 2009 


\title{
Supplement for "Identification and Estimation of Games with Incomplete Information Using Excluded Regressors"
}

\author{
Arthur Lewbel, Boston College
}

Xun Tang, University of Pennsylvania

Original: November 25, 2010. Revised: February 28, 2013

\section{Proofs of Asymptotics}

Assumptions used in the results below are all listed in the main text of Lewbel and Tang (2012).

\subsection{Limiting distribution of $\sqrt{G}\left(\hat{A}_{i}-A_{i}\right)$}

To simplify notation, for a pair of players $i$ and $j=3-i$, we define the following shorthands: $a=\gamma_{0}^{*}, b=\gamma_{i}^{*}, c=\gamma_{j}^{*}, d=\gamma_{0, i}^{*}, e=\gamma_{0, j}^{*}, f=\gamma_{i, i}^{*}, g=\gamma_{i, j}^{*}, h=\gamma_{j, i}^{*}$ and $k=\gamma_{j, j}^{*}$, with all these functions evaluated at $x$. As in the text, let $w_{g}, w_{x}$ denote $1\left\{x_{g} \in \omega\right\}$ and $1\{x \in \omega\}$ respectively. For any $x \in \Omega_{X}$, define a linear functional of $\gamma_{(1)}$ :

$$
\begin{aligned}
M_{A}^{i}\left(x ; \gamma_{(1)}\right) \equiv & {\left[\gamma_{(1)}(x)\right]^{\prime} \tilde{D}_{i, A}(x), \text { where } } \\
\tilde{D}_{i, A}\left(x ; \gamma_{(1)}^{*}\right) \equiv & {\left[\begin{array}{c}
\frac{-1}{a^{2}(c e-a k)^{2}}\left(\begin{array}{c}
c^{2} f e^{2}+a^{2} f k^{2}-c^{2} d g e-2 a b d k^{2}-a^{2} g h k \\
-b c h e^{2}+b c d k e-2 a c f k e+2 a b h k e+2 a c d g k
\end{array}\right), . . \\
\frac{1}{a(a k-c e)}(h e-d k), \frac{b e-a g}{a(c e-a k)^{2}}(h e-d k), \frac{1}{a(a k-c e)}(c g-b k), . . \\
-\frac{1}{a^{2} e}\left(b d-a f-\frac{(b e-a g)(c d-a h)}{a k-c e}\right), \frac{1}{a}, \frac{1}{a(a k-c e)}(c d-a h), . . \\
\frac{1}{a(a k-c e)}(b e-a g), \frac{b e-a g}{a(a k-c e)^{2}}(c d-a h)
\end{array}\right] }
\end{aligned}
$$

Lemma 1.1 Suppose Assumptions $S, K, B$ and $D$-(i),(ii) hold. Then for $i=1,2$,

$$
\frac{1}{G} \sum_{g} w_{g}\left[m_{A}^{i}\left(x_{g} ; \hat{\gamma}_{(1)}\right)-m_{A}^{i}\left(x_{g} ; \gamma_{(1)}^{*}\right)\right]=\int w_{x} M_{A}^{i}\left(x ; \hat{\gamma}_{(1)}-\gamma_{(1)}^{*}\right) d F_{X}^{*}+o_{p}\left(G^{-1 / 2}\right)
$$

Proof of Lemma 1.1. Consider $i=1$. Under D-(i), (ii), there exists some $b(x)$ such that $E[b(x)]<$ $\infty$ and

$$
\begin{aligned}
& \left\|w_{g}\left[m_{A}^{i}\left(x_{g} ; \hat{\gamma}_{(1)}\right)-m_{A}^{i}\left(x_{g} ; \gamma_{(1)}^{*}\right)-\left(\hat{\gamma}_{(1)}\left(x_{g}\right)-\gamma_{(1)}^{*}\left(x_{g}\right)\right)^{\prime} \tilde{D}_{i, A}\left(x_{g}\right)\right]\right\| \\
\leq & b\left(x_{g}\right) \sup _{x \in \omega}\left\|\hat{\gamma}_{(1)}-\gamma_{(1)}^{*}\right\|^{2}
\end{aligned}
$$


Then Lemma 8.10 in Newey and McFadden (1994) applies under S-(i),(ii),(iii), K and B, with the order of derivatives being 1 in the definition of the Sobolev Norm and with the dimension of continuous coordinates being $J_{c}$. Hence $\sup _{x \in \omega}\left\|\hat{\gamma}_{(1)}-\gamma_{(1)}^{*}\right\|=o_{p}\left(G^{-1 / 4}\right)$, and

$$
\frac{1}{G}\left\|\begin{array}{l}
\sum_{g} w_{g}\left[m_{A}^{i}\left(x_{g} ; \hat{\gamma}_{(1)}\right)-m_{A}^{i}\left(x_{g} ; \gamma_{(1)}^{*}\right)\right] \\
-\sum_{g} w_{g}\left(\hat{\gamma}_{(1)}\left(x_{g}\right)-\gamma_{(1)}^{*}\left(x_{g}\right)\right)^{\prime} \tilde{D}_{i, A}\left(x_{g}\right)
\end{array}\right\|=o_{p}\left(G^{-1 / 2}\right) .
$$

Next, define $\bar{\gamma}_{(1)} \equiv E\left[\hat{\gamma}_{(1)}\right]$ and note:

$$
\begin{aligned}
& G^{-1} \sum_{g} w_{g}\left(\hat{\gamma}_{(1)}\left(x_{g}\right)-\gamma_{(1)}^{*}\left(x_{g}\right)\right)^{\prime} \tilde{D}_{i, A}\left(x_{g}\right)-\int w_{x}\left(\hat{\gamma}_{(1)}(x)-\gamma_{(1)}^{*}(x)\right)^{\prime} \tilde{D}_{i, A}(x) d F_{X}^{*} \\
= & G^{-1} \sum_{g} w_{g}\left(\hat{\gamma}_{(1)}\left(x_{g}\right)-\bar{\gamma}_{(1)}\left(x_{g}\right)\right)^{\prime} \tilde{D}_{i, A}\left(x_{g}\right)-\int w_{x}\left(\hat{\gamma}_{(1)}(x)-\bar{\gamma}_{(1)}(x)\right)^{\prime} \tilde{D}_{i, A}(x) d F_{X}^{*} \\
+ & G^{-1} \sum_{g} w_{g}\left(\bar{\gamma}_{(1)}\left(x_{g}\right)-\gamma_{(1)}^{*}\left(x_{g}\right)\right)^{\prime} \tilde{D}_{i, A}\left(x_{g}\right)-\int w_{x}\left(\bar{\gamma}_{(1)}(x)-\gamma_{(1)}^{*}(x)\right)^{\prime} \tilde{D}_{i, A}(x) d F_{X}^{*}
\end{aligned}
$$

Let $a_{G}\left(z_{g}, z_{s}\right) \equiv$

$$
w_{g}\left[\begin{array}{l}
K_{\sigma}\left(x_{s}-x_{g}\right), d_{s, 1} K_{\sigma}\left(x_{s}-x_{g}\right), d_{s, 2} K_{\sigma}\left(x_{s}-x_{g}\right), \ldots \\
K_{\sigma, 1}\left(x_{s}-x_{g}\right), d_{s, 1} K_{\sigma, 1}\left(x_{s}-x_{g}\right), d_{s, 2} K_{\sigma, 1}\left(x_{s}-x_{g}\right), \ldots \\
K_{\sigma, 2}\left(x_{s}-x_{g}\right), d_{s, 1} K_{\sigma, 2}\left(x_{s}-x_{g}\right), d_{s, 2} K_{\sigma, 2}\left(x_{s}-x_{g}\right)
\end{array}\right]^{\prime} \tilde{D}_{i, A}\left(x_{g}\right) .
$$

Let $a_{G, 1}\left(z_{g}\right) \equiv \int a\left(z_{g}, z\right) d F_{Z}^{*}(z)$ and $a_{G, 2}\left(z_{s}\right) \equiv \int a_{G}\left(z, z_{s}\right) d F_{Z}^{*}(z)$. The first difference on the right-hand side of (2) takes the form of a $V$-statistic:

$$
G^{-2} \sum_{g} \sum_{s} a_{G}\left(z_{g}, z_{s}\right)-G^{-1} \sum_{g} a_{G, 1}\left(z_{g}\right)-G^{-1} \sum_{g} a_{G, 2}\left(z_{g}\right)+E\left[a_{G, 1}(z)\right]
$$

By Assumption D-(i) and boundedness of kernels in K, $\left\|w_{x} M_{A}^{i}\left(x ; \gamma_{(1)}\right)\right\| \leq c(x) \sup _{x \in \omega}\left\|\gamma_{(1)}\right\|$ for some non-negative function $c$ such that $E\left[c(X)^{2}\right]<\infty$. Consequently, $E\left[\left\|a_{G}\left(Z_{g}, Z_{g}\right)\right\|\right] \leq$ $\sigma^{-J} E\left[c\left(X_{g}\right)\right] C_{1}$ and $\left\{E\left[\left\|a_{G}\left(Z_{g}, Z_{s}\right)\right\|^{2}\right]\right\}^{1 / 2} \leq \sigma^{-J}\left\{E\left[c^{2}\left(X_{g}\right)\right]\right\}^{1 / 2} C_{2}$, where $C_{1}, C_{2}$ are finite positive constants. Since $G^{-1} \sigma^{-J} \rightarrow 0$, it follows from Lemma 8.4 in Newey and McFadden (1994) that the first difference is $o_{p}\left(G^{-1 / 2}\right)$.

We now turn to the second difference in (2). Since $\left\|w_{x} M_{A}^{i}\left(x ; \gamma_{(1)}\right)\right\| \leq c(x) \sup _{x \in \omega}\left\|\gamma_{(1)}\right\|$ and $E\left[c(X)^{2}\right]<\infty$

$$
E\left[\left\|w_{X} M_{A}^{i}\left(X ; \bar{\gamma}_{(1)}-\gamma_{(1)}^{*}\right)\right\|^{2}\right] \leq E\left[c(X)^{2}\right] \sup _{x \in \omega}\left\|\bar{\gamma}_{(1)}-\gamma_{(1)}^{*}\right\|^{2}
$$

By Lemma 8.9 in Newey and McFadden (1994) and Hardle and Linton (1994), we have $\sup _{x \in \omega}\left\|\bar{\gamma}_{(1)}-\gamma_{(1)}^{*}\right\|^{2}$ $=O\left(\sigma^{m}\right)$ under conditions in $\mathrm{S}, \mathrm{K}$ and $\mathrm{B}$. Thus, as $G \rightarrow+\infty$,

$$
E\left[\sup _{x \in \omega}\left\|w_{X} M_{A}^{i}\left(X ; \bar{\gamma}_{(1)}-\gamma_{(1)}^{*}\right)\right\|^{2}\right] \rightarrow 0
$$


Then by the Chebychev's Inequality, the second difference in $(2)$ is $o_{p}\left(G^{-1 / 2}\right)$. This proves the lemma. Q.E.D.

Lemma 1.2 Suppose Assumptions $S, K, B, D$ and $W$ hold. Then

$$
\int w_{x} M_{A}^{i}\left(x ; \hat{\gamma}_{(1)}-\gamma_{(1)}^{*}\right) d F_{X}^{*}=G^{-1} \sum_{g} \tilde{\nu}_{A}^{i}\left(z_{g}\right)+o_{p}\left(G^{-1 / 2}\right),
$$

where $\tilde{\nu}_{A}^{i}\left(z_{g}\right) \equiv \psi_{A}^{i}\left(x_{g}\right) d_{g}-E\left[\psi_{A}^{i}(X) D\right]$.

Proof of Lemma 1.2. For $i=1,2$ and $j=3-i$, by definition of $M_{A}^{i}$,

$$
\begin{aligned}
& \int w_{x} M_{A}^{i}\left(x ; \gamma_{(1)}\right) d F_{X}^{*} \\
= & \int w_{x}\left[\begin{array}{c}
\gamma_{0}(x) \\
\gamma_{i}(x) \\
\gamma_{j}(x)
\end{array}\right]^{\prime}\left[\begin{array}{c}
\tilde{D}_{i, A, 1}(x) \\
\tilde{D}_{i, A, 2}(x) \\
D_{i, A, 3}(x)
\end{array}\right]+w_{x}\left[\begin{array}{c}
\gamma_{0, i}(x), \gamma_{0, j}(x), . . \\
\gamma_{i, i}(x), \gamma_{i, j}(x), . . \\
\gamma_{j, i}(x), \gamma_{j, j}(x) \\
1-b y-6
\end{array}\right]\left[\begin{array}{c}
\tilde{D}_{i, A, 4}(x) \\
\vdots \\
\tilde{D}_{i, A, 9}(x) \\
6-b y-1
\end{array}\right] d x .
\end{aligned}
$$

Applying integration-by-parts to the second inner product in the integrand of (3) and using the convexity of $\omega$ (Assumption $\mathrm{W}$ ), we have

$$
\int w_{x} M_{A}^{i}\left(x ; \gamma_{(1)}\right) d F_{X}^{*}=\int\left[\begin{array}{c}
\gamma_{0}(x) \\
\gamma_{i}(x) \\
\gamma_{j}(x)
\end{array}\right]^{\prime}\left[\begin{array}{c}
\psi_{A, 0}^{i}(x) \\
\psi_{A, i}^{i}(x) \\
\psi_{A, j}^{i}(x)
\end{array}\right] d x
$$

for any $\gamma_{(1)}$ that is continuously differentiable in excluded regressors $x_{e}$. The functions $\psi_{A, 0}^{i}, \psi_{A, i}^{i}, \psi_{A, j}^{i}$ are defined in the text. (See the web appendix of this supplement for detailed derivations.) These three functions only depend on $\gamma_{(1)}^{*}$ but not $\gamma_{(1)}$. By conditions in S and K, both $\hat{\gamma}_{(1)}$ and $\gamma_{(1)}^{*}$ are continuously differentiable. For the rest of the proof, we focus on the case where all coordinates in $\tilde{x}$ are continuously distributed. ${ }^{1}$ It follows that:

$$
\begin{aligned}
& \int w_{x} M_{A}^{i}\left(x ; \hat{\gamma}_{(1)}-\gamma_{(1)}^{*}\right) d F_{X}^{*}=\int \sum_{s=0, i, j} \psi_{A, s}^{i}(x)\left[\hat{\gamma}_{s}(x)-\gamma_{s}^{*}(x)\right] d x \\
= & G^{-1} \sum_{g} \int \sum_{s=0, i, j} \psi_{A, s}^{i}(x) d_{g, s} K_{\sigma}\left(x-x_{g}\right) d x-E\left[\psi_{A}^{i}(X) D\right] \\
= & G^{-1} \sum_{g} \int\left(\sum_{s=0, i, j} \psi_{A, s}^{i}(x) d_{g, s}-\mu\right) K_{\sigma}\left(x-x_{g}\right) d x \equiv \int\left(\sum_{s=0, i, j} \psi_{A, s}^{i}(x) d_{s}-\mu\right) d \hat{F}_{Z}
\end{aligned}
$$

where $d_{g, 0} \equiv 1, \mu \equiv E\left[\psi_{A}^{i}(X) D\right]$, and $\hat{F}_{Z}$ is a "smoothed-version" of empirical distribution of $z \equiv$ $\left(x, d_{1}, d_{2}\right)$ with the expectation of $a(z)$ with respect to $\hat{F}_{Z}$ equal to $G^{-1} \sum_{g} \int a\left(x, d_{1, g}, d_{2, g}\right) K_{\sigma}(x-$

\footnotetext{
${ }^{1}$ General cases involving discrete coordinates only add to complex notations, but do not require any changes in the the arguments. For example, $\int \psi_{A}^{i}(x) d_{g} K_{\sigma}\left(x-x_{g}\right) d x$ in the proof would be replaced by $\sum_{\tilde{x}_{d}} \int \psi_{A}^{i}\left(x_{c}, \tilde{x}_{d}\right) d_{g} K_{\sigma}\left(x_{c}-\right.$ $\left.x_{g, c}\right) d x_{c} 1\left\{\tilde{x}_{g, d}=\tilde{x}_{d}\right\}$. Likewise, $\int K_{\sigma}\left(x-x_{g}\right) d x$ would be replaced by $\sum_{\tilde{x}_{d}} \int K_{\sigma}\left(x_{c}-x_{g, c}\right) d x_{c} 1\left\{\tilde{x}_{g, d}=\tilde{x}_{d}\right\}$ (which equals 1).
} 
$\left.x_{g}\right) d x$. By construction, $\frac{1}{G} \sum_{g} \int \mu K_{\sigma}\left(x-x_{g}\right) d x=\mu$. Hence by definition:

$$
\begin{aligned}
& \int\left(\sum_{s=0, i, j} \psi_{A, s}^{i}(x) d_{s}-\mu\right) d \hat{F}_{Z}-G^{-1} \sum_{g}\left(\sum_{s=0, i, j} \psi_{A, s}^{i}\left(x_{g}\right) d_{s, g}-\mu\right) \\
= & \int\left(\sum_{s=0, i, j} \psi_{A, s}^{i}(x) d_{s}\right) d \hat{F}_{Z}-G^{-1} \sum_{g}\left(\sum_{s=0, i, j} \psi_{A, s}^{i}\left(x_{g}\right) d_{s, g}\right) \\
= & G^{-1} \sum_{g}\left[\sum_{s=0, i, j} d_{s, g}\left(\int \psi_{A, s}^{i}(x) K_{\sigma}\left(x-x_{g}\right) d x-\psi_{A, s}^{i}\left(x_{g}\right)\right)\right]
\end{aligned}
$$

It remains to show that the r.h.s. of $(4)$ is $o_{p}\left(G^{-1 / 2}\right)$. First, note

$$
\begin{aligned}
& \left\|\sqrt{G} E\left[\sum_{s=0, i, j} d_{s, g}\left(\int \psi_{A, s}^{i}(x) K_{\sigma}\left(x-x_{g}\right) d x-\psi_{A, s}^{i}\left(x_{g}\right)\right)\right]\right\| \\
= & \sqrt{G}\left\|\sum_{s=0, i, j}\left(\int\left(\int \psi_{A, s}^{i}(x) K_{\sigma}(x-\hat{x}) d x\right) \gamma_{s}^{*}(\hat{x}) d \hat{x}-\int \psi_{A, s}^{i}(x) \gamma_{s}^{*}(x) d x\right)\right\| \\
= & \sqrt{G}\left\|\sum_{s=0, i, j}\left(\iint \psi_{A, s}^{i}(\hat{x}+\sigma u) K(u) \gamma_{s}^{*}(\hat{x}) d u d \hat{x}-\int \psi_{A, s}^{i}(x) \gamma_{s}^{*}(x) d x\right)\right\| \\
= & \sqrt{G}\left\|\sum_{s=0, i, j}\left(\int \psi_{A, s}^{i}(x)\left(\int K(u) \gamma_{s}^{*}(x-\sigma u) d u\right) d x-\int \psi_{A, s}^{i}(x) \gamma_{s}^{*}(x) d x\right)\right\|
\end{aligned}
$$

where the equalities follows from a change-of -variable between $x$ and $u \equiv \frac{x-\hat{x}}{\sigma}$ first, and then another change-of-variable between $\hat{x}$ and $x=\hat{x}+\sigma u$. (Such operations can be done due to the smoothness conditions on $\gamma_{(1)}^{*}$ and the forms of $\psi_{A, s}^{i}$ for $s=0, i, j$.) Next, using $\int K(u) d u=1$ and re-arranging terms, we can write (5) as:

$$
\begin{aligned}
& \sqrt{G}\left\|\sum_{s=0, i, j} \int \psi_{A, s}^{i}(x)\left\{\int\left[\gamma_{s}^{*}(x-\sigma u)-\gamma_{s}^{*}(x)\right] K(u) d u\right\} d x\right\| \\
\leq & \sqrt{G} \int\left\|\psi_{A}^{i}(x)\right\|\left\|\int\left[\gamma^{*}(x-\sigma u)-\gamma^{*}(x)\right] K(u) d u\right\| d x,
\end{aligned}
$$

where the inequality follows from the Cauchy-Schwarz Inequality. By definition, for $s=0, i, j$ and any fixed $x$,

$$
E\left[\hat{\gamma}_{s}(x)\right]=E\left[D_{s} K_{\sigma}\left(x-X_{g}\right)\right]=\int E\left[D_{s} \mid x_{g}\right] K_{\sigma}\left(x-x_{g}\right) \gamma_{0}^{*}\left(x_{g}\right) d x_{g}=\int \gamma_{s}^{*}(x-\sigma u) K(u) d u
$$

where the last equality follows from a change-of-variable between $x_{g}$ and $u \equiv \frac{x-x_{g}}{\sigma}$. (Recall $D_{0} \equiv$ 1.) Under S-(i) and K-(i) (smoothness of $\gamma^{*}$ and the kernel functions) and K-(ii) (on the order of the kernel), $\sup _{x \in \omega}\left\|E\left[\hat{\gamma}(x)-\gamma^{*}(x)\right]\right\|=O\left(\sigma^{m}\right)$. Under the boundedness condition in D-(i), $\int \sup _{x \in \omega}\left\|\psi_{A}^{i}(x)\right\| d x<\infty$ and the r.h.s. of the inequality in (6) is bounded above by $\sqrt{G} \sigma^{m} \tilde{C}$, with $\tilde{C}$ being some finite positive constant. Since $\sqrt{G} \sigma^{m} \longrightarrow 0$, it follows that

$$
E\left\|\left[\sum_{s=0, i, j} d_{g, s}\left(\int \psi_{A, s}^{i}(x) K_{\sigma}\left(x-x_{g}\right) d x-\psi_{A, s}^{i}\left(x_{g}\right)\right)\right]\right\|=o_{p}\left(G^{-1 / 2}\right)
$$

By definition of $\psi_{A}^{i}$ and the smoothness condition in D-(i), $\psi_{A}^{i}$ is continuous in $x$ almost everywhere, and $\psi_{A, s}^{i}(x+\sigma u) \rightarrow \psi_{A, s}^{i}(x)$ for almost all $x$ and $u$ and $s=0, i, j$. By condition D-(iii), for $\sigma$ small 
enough, $\psi_{A, s}^{i}(x+\sigma u)$ is bounded above by some function of $x$ for almost all $x$ and $s=0, i, j$ on the support of $K($.$) . It then follows from the Dominated Convergence Theorem that$

$$
\int \psi_{A}^{i}(x+\sigma u) K(u) d u \rightarrow \int \psi_{A}^{i}(x) K(u) d u=\psi_{A}^{i}(x)
$$

for almost all $x$ as $\sigma \rightarrow 0$, where $\psi_{A}^{i}=\left[\psi_{A, 0}^{i}, \psi_{A, i}^{i}, \psi_{A, j}^{i}\right]$. Under the boundedness conditions of the kernel in $\mathrm{K}$ and $\mathrm{S}$-(iii), the Dominated Convergence Theorem applies again to give

$$
E\left[\left\|\int \psi_{A}^{i}(\tilde{x}) K_{\sigma}(\tilde{x}-x) d \tilde{x}-\psi_{A}^{i}(x)\right\|^{4}\right] \rightarrow 0 .
$$

It then follows from the Cauchy-Schwartz Inequality that

$$
E\left[\|D\|^{2}\left\|\int \psi_{A}^{i}(\tilde{x}) K_{\sigma}(\tilde{x}-x) d \tilde{x}-\psi_{A}^{i}(x)\right\|^{2}\right] \rightarrow 0 .
$$

Hence both the mean and variance of the product of $\sqrt{G}$ and the left-hand side of (4) converge to 0 . It then follows from Chebyshev's Inequality that this product converges in probability to 0 . Q.E.D.

It follows from Lemma 1.1 and Lemma 1.2 that

$$
\frac{1}{G} \sum_{g} w_{g} m_{A}^{i}\left(x_{g} ; \hat{\gamma}_{(1)}\right)=\frac{1}{G} \sum_{g}\left[w_{g} m_{A}^{i}\left(x_{g} ; \boldsymbol{\gamma}_{(1)}^{*}\right)+\tilde{\nu}_{A}^{i}\left(z_{g}\right)\right]+o_{p}\left(G^{-1 / 2}\right),
$$

which implies

$$
\hat{A}^{i}-A^{i}=\frac{1}{G} \sum_{g}\left\{w_{g} m_{A}^{i}\left(x_{g} ; \boldsymbol{\gamma}_{(1)}^{*}\right)-E\left[w_{X} m_{A}^{i}\left(X ; \boldsymbol{\gamma}_{(1)}^{*}\right)\right]+\tilde{\nu}_{A}^{i}\left(z_{g}\right)\right\}+o_{p}\left(G^{-1 / 2}\right) .
$$

Note the summand (the expression in $\{\ldots\}$ ) has zero mean by construction, and has a finite second moment under the boundedness conditions in Assumptions S and D. Hence Lemma 1 in Section 6.1 of Lewbel and Tang (2012) follows from the Central Limit Theorem.

\subsection{Limiting distribution of $\sqrt{G}\left(\hat{\Delta}^{i}-\Delta^{i}\right)$}

We now examine the limiting behavior of $\sqrt{G}\left(\hat{\Delta}^{i}-\Delta^{i}\right)$. Rewrite the definition of $\hat{\Delta}^{i}$ in equation (18) of Lewbel and Tang (2012) as

$$
\hat{\Delta}^{i} \equiv(\hat{\rho})^{-1}\left[\frac{1}{G} \sum_{g} w_{g} m_{\Delta}^{i}\left(x_{g} ; \hat{\gamma}_{(1)}\right)\right]
$$

where $\hat{\rho} \equiv\left[G^{-1} \sum_{g} w_{g}\right]^{-1}$. Furthermore, let $w_{x} \equiv 1\{x \in \omega\}$ as before, and define $\rho_{0} \equiv E[1\{X \in$ $\omega\}]=\operatorname{Pr}(X \in \omega)$. The first step in Lemma 2.1 below is to describe the impact of replacing $\hat{\rho}$ with the true population moment $\rho_{0}$ in $(7)$. 
Lemma 2.1 Suppose Assumptions S, K, B and D hold. Then:

$$
\hat{\Delta}^{i}=G^{-1} \sum_{g}\left[\rho_{0}^{-1} w_{g} m_{\Delta}^{i}\left(x_{g} ; \hat{\gamma}_{(1)}\right)-\mathcal{M}_{i, \rho}^{*}\left(w_{g}-\rho_{0}\right)\right]+o_{p}\left(G^{-1 / 2}\right)
$$

where

$$
\mathcal{M}_{i, \rho}^{*} \equiv \rho_{0}^{-2} E\left[w_{X} m_{\Delta}^{i}\left(X ; \gamma_{(1)}^{*}\right)\right]
$$

Proof of Lemma 2.1. Apply the Taylor's Expansion to (7) around $\rho_{0}$, we get:

$$
\hat{\Delta}^{i}=\rho_{0}^{-1}\left[\frac{1}{G} \sum_{g} w_{g} m_{\Delta}^{i}\left(x_{g} ; \hat{\gamma}_{(1)}\right)\right]-\hat{\mathcal{M}}_{i, \rho}\left(\frac{1}{G} \sum_{g} 1\left\{x_{g} \in \omega\right\}-\rho_{0}\right)
$$

where

$$
\hat{\mathcal{M}}_{i, \rho} \equiv \tilde{\rho}^{-2}\left[\frac{1}{G} \sum_{g} w_{g} m_{\Delta}^{i}\left(x_{g} ; \hat{\gamma}_{(1)}\right)\right]
$$

and $\tilde{\rho}$ lies on a line segment between $\hat{\rho}$ and $\rho_{0}$. By the Law of Large Numbers, $\hat{\rho} \stackrel{p}{\longrightarrow} \rho_{0}$ and therefore $\tilde{\rho} \stackrel{p}{\longrightarrow} \rho_{0}$. Under Assumptions in S, K and B, $\sup _{x \in \omega}\left\|\hat{\gamma}_{(1)}-\gamma_{(1)}^{*}\right\|=o_{p}\left(G^{-1 / 4}\right)$. By definition of $m_{\Delta}^{i}$, it can be shown that $w_{X} m_{\Delta}^{i}\left(X ; \gamma_{(1)}^{*}\right)$ is continuous at $\gamma_{(1)}^{*}$ with probability one. Lemma 4.3 in Newey and McFadden (1994) then implies that, under Assumption D-(iv), $\frac{1}{G} \sum_{g} w_{g} m_{\Delta}^{i}\left(x_{g} ; \hat{\gamma}_{(1)}\right) \stackrel{p}{\longrightarrow} E\left[w_{X} m_{\Delta}^{i}\left(X ; \gamma_{(1)}^{*}\right)\right]$. By the Slutsky Theorem, $\hat{\mathcal{M}}_{i, \rho}=\mathcal{M}_{i, \rho}^{*}+o_{p}(1)$. Furthermore, $\frac{1}{G} \sum_{g} 1\left\{x_{g} \in \omega\right\}-\rho_{0}=O_{p}\left(G^{-1 / 2}\right)$ by the Central Limit Theorem. It then follows that

$$
\hat{\Delta}^{i}=\rho_{0}^{-1}\left[\frac{1}{G} \sum_{g} w_{g} m_{\Delta}^{i}\left(x_{g} ; \hat{\gamma}_{(1)}\right)\right]-\mathcal{M}_{i, \rho}^{*}\left(\frac{1}{G} \sum_{g} 1\left\{x_{g} \in \omega\right\}-\rho_{0}\right)+o_{p}\left(G^{-1 / 2}\right)
$$

This proves the lemma. Q.E.D.

The remaining steps for deriving the limiting distribution of $\sqrt{G}\left(\hat{\Delta}^{i}-\Delta^{i}\right)$ are similar to those used for $\sqrt{G}\left(\hat{A}^{i}-A^{i}\right)$. Let $a, b, c, d, e, f, g, h, k$ be the same short-hands as defined above (i.e. shorthands for coordinates in $\gamma_{(1)}^{*}$ at some $\left.x\right)$. The dependence of these short-hands on $x$ are suppressed in notations. First, for any $x \in \Omega_{X}$, define a linear functional of $\gamma_{(1)}$ :

$$
\begin{aligned}
& M_{\Delta}^{i}\left(x ; \gamma_{(1)}\right) \equiv\left[\gamma_{(1)}(x)\right]^{\prime} \tilde{D}_{i, \Delta}(x), \text { where } \\
& \tilde{D}_{i, \Delta}\left(x ; \gamma_{(1)}^{*}\right) \equiv\left[\begin{array}{c}
\frac{-b^{2} h e^{2}-a^{2} g^{2} h+b^{2} d k e+2 a c d g^{2}+a^{2} f g k+b c f e^{2}-b c d g e-2 a c f g e+2 a b g h e-2 a b d g k}{(-c f e+b h e+c d g-a g h-b d k+a f k)^{2}}, \\
\frac{a(c e-a k)(f e-d g)}{(c f e-b h e-c d g+a g h+b d k-a f k)^{2}}, \frac{-a(b e-a g)(f e-d g)}{(c f e-b h e-c d g+a g h+b d k-a f k)^{2}}, \frac{a(b e-a g)(c g-b k)}{(c f e-b h e-c d g+a g h+b d k-a f k)^{2}}, \\
\frac{-a(b d-a f)(c g-b k)}{(c d g-a g h-b d k+a f k-c f e+b h)^{2}}, \frac{a(c e-a k)(b d-a f)}{(c f e-b h e-c d g+a g h+b d k-a f k)^{2}}, \frac{a f f e-b h e-c d g+a g h+b d k-a f k)^{2}}{(c f e-a g)(c e-a k)}, \\
\frac{a(b e-a g)^{2}}{(c f e-b h e-c d g+a g h+b d k-a f k)^{2}}, \frac{-a(b e-a g)(b d-a f)}{(c f e-b h e-c d g+a g h+b d k-a f k)^{2}}
\end{array}\right]^{\prime} .
\end{aligned}
$$

The detailed forms of $\tilde{D}_{i, \Delta}^{(s)}$ (partial derivatives of $\tilde{D}_{i, \Delta}^{(s)}$ with respect to special regressors $X_{s}$ ) are given in the web appendix to economize space here. The correction terms $\psi_{\Delta}^{i} \equiv\left[\psi_{\Delta, 0}^{i}, \psi_{\Delta, i}^{i}, \psi_{\Delta, j}^{i}\right]$ are defined in the text. 
Lemma 2.2 Suppose Assumptions $S, K, B, D$ and $W$ hold. Then for $i=1,2$,

$$
\frac{1}{G} \sum_{g} w_{g} m_{\Delta}^{i}\left(x_{g} ; \hat{\gamma}_{(1)}\right)=\frac{1}{G} \sum_{g}\left[w_{g} m_{\Delta}^{i}\left(x_{g} ; \gamma_{(1)}^{*}\right)+\tilde{\nu}_{\Delta}\left(z_{g}\right)\right]+o_{p}\left(G^{-1 / 2}\right)
$$

where $\tilde{\nu}_{\Delta}^{i}\left(z_{g}\right) \equiv \psi_{\Delta}^{i}\left(x_{g}\right) d_{g}-E\left[\psi_{\Delta}^{i}(X) D\right]$.

The proof of Lemma 2.2 uses the same arguments as those for Lemma 1.1 and Lemma 1.2. We provide some additional details for derivations in the web appendix of this article. Combining results in (8) and (9), we have

$$
\begin{aligned}
\hat{\Delta}^{i}-\Delta^{i} & =G^{-1} \sum_{g}\left[w_{g} m_{\Delta}^{i}\left(x_{g} ; \gamma_{(1)}^{*}\right) / \rho_{0}+\tilde{\nu}_{\Delta}^{i}\left(z_{g}\right) / \rho_{0}-\Delta^{i}-\mathcal{M}_{i, \rho}^{*}\left(w_{g}-\rho_{0}\right)\right]+o_{p}\left(G^{-1 / 2}\right) \\
& =\frac{1}{\rho_{0}} \frac{1}{G} \sum_{g}\left[w_{g} m_{\Delta}^{i}\left(x_{g} ; \gamma_{(1)}^{*}\right)+\tilde{\nu}_{\Delta}^{i}\left(z_{g}\right)-\rho_{0} \Delta^{i}-\Delta^{i}\left(w_{g}-\rho_{0}\right)\right]+o_{p}\left(G^{-1 / 2}\right)
\end{aligned}
$$

where the summand in the square bracket has zero mean by construction, and have a finite second moment under the boundedness assumptions in S. Since $\Delta^{i}$ is a constant, it follows from the Central Limit Theorem that:

$$
\sqrt{G}\left(\hat{\Delta}^{i}-\Delta^{i}\right) \stackrel{d}{\longrightarrow} \mathcal{N}\left(0, \rho_{0}^{-2} \operatorname{Var}\left[w_{X}\left(m_{\Delta}^{i}\left(X ; \gamma_{(1)}^{*}\right)-\Delta^{i}\right)+\tilde{\nu}_{\Delta}^{i}(Z)\right]\right) .
$$

\subsection{Limiting distribution of $\sqrt{G}\left(\hat{\beta}_{i}-\beta_{i}\right)$}

Lemma 3.1 Suppose Assumptions $S^{\prime}-(a),(b), K, B$ and $D^{\prime}-(i)$ hold. Then:

$$
\frac{1}{G} \sum_{g} m_{B}^{i}\left(z_{g} ; \hat{\delta}_{i}, \hat{\gamma}_{(1, i)}\right)=\frac{1}{G} \sum_{g}\left[m_{B}^{i}\left(z_{g} ; \delta_{i}, \hat{\gamma}_{(1, i)}\right)+\mathcal{M}_{i, \delta}^{*} \Psi_{\delta}^{i}\left(z_{g}\right)\right]+o_{p}\left(G^{-1 / 2}\right)
$$

where

$$
\begin{aligned}
\mathcal{M}_{i, \delta}^{*} & \equiv E\left[\tilde{D}_{i, B, \delta}\left(Z ; \delta_{i}, \gamma_{(1, i)}^{*}\right)\right] \text { and } \\
\Psi_{\delta}^{i}\left(z_{g}\right) & \equiv-\frac{1}{\rho_{0}}\left[w_{g} m_{\Delta}^{i}\left(x_{g} ; \gamma_{(1, i)}^{*}\right)+\tilde{\nu}_{\Delta}^{i}\left(z_{g}\right)-\rho_{0} \Delta^{i}-\Delta^{i}\left(w_{g}-\rho_{0}\right)\right]
\end{aligned}
$$

Proof of Lemma 3.1. By definition and our choice of the distribution function, conditions in S' and $\mathrm{K}, m_{B}^{i}\left(z ; \delta_{i}, \hat{\gamma}_{(1, i)}\right)$ is continuously differentiable in $\delta_{i}$. By the Mean Value Theorem,

$$
m_{B}^{i}\left(z_{g} ; \hat{\delta}_{i}, \hat{\gamma}_{(1, i)}\right)=m_{B}^{i}\left(z_{g} ; \delta_{i}, \hat{\gamma}_{(1, i)}\right)+\tilde{D}_{i, B, \delta}\left(z_{g} ; \bar{\delta}_{i}, \hat{\gamma}_{(1, i)}\right)\left(\hat{\delta}_{i}-\delta_{i}\right)
$$

where $\tilde{D}_{i, B, \delta}\left(z_{g} ; \bar{\delta}_{i}, \hat{\gamma}_{(1, i)}\right)$ is the partial derivative of $m_{B}^{i}$ with respect to the interaction effect at $\bar{\delta}_{i}$, which is an intermediate value between $\hat{\delta}_{i}$ and $\delta_{i}$. Hence

$$
\begin{aligned}
& \frac{1}{\sqrt{G}} \sum_{g} m_{B}^{i}\left(z_{g} ; \hat{\delta}_{i}, \hat{\gamma}_{(1, i)}\right) \\
= & \frac{1}{\sqrt{G}} \sum_{g} m_{B}^{i}\left(z_{g} ; \delta_{i}, \hat{\gamma}_{(1, i)}\right)+\frac{1}{G} \sum_{g} \tilde{D}_{i, B, \delta}\left(z_{g} ; \bar{\delta}_{i}, \hat{\gamma}_{(1, i)}\right) \sqrt{G}\left(\hat{\delta}_{i}-\delta_{i}\right) .
\end{aligned}
$$


With $\alpha_{i}=-1$, it follows from (10) that $\sqrt{G}\left(\hat{\delta}_{i}-\delta_{i}\right)=G^{-1 / 2} \sum_{g} \Psi_{\delta}^{i}\left(z_{g}\right)+o_{p}(1)$, where the influence function is defined as in (11). (Recall that $\delta_{i}=\alpha_{i} \Delta^{i}$.) Let $\sup _{x \in \Omega_{X}}\|\cdot\|$ (where $\|\cdot\|$ is the Euclidean norm) be the norm for the space of generic $\gamma_{(1, i)}$ that are continuously differentiable in $x_{c}$ up to an order of $\bar{m} \geq 0$ given all $\tilde{x}_{d}$. Under conditions in S' and $\mathrm{K}, \tilde{D}_{i, B, \delta}\left(z ; \delta_{i}, \gamma_{(1, i)}\right)$ is continuous in $\delta_{i}, \gamma_{(1, i)}$ with probability 1 . Note $\bar{\delta}_{i} \stackrel{p}{\longrightarrow} \delta_{i}$ as a consequence of $\hat{\delta}_{i} \stackrel{p}{\longrightarrow} \delta_{i}$. Under conditions in S'-(a),(b), K, and B, $\sup _{x \in \Omega_{X}}\left\|\hat{\gamma}_{(1, i)}-\gamma_{(1, i)}^{*}\right\| \stackrel{p}{\longrightarrow} 0$ at the rate faster than $G^{1 / 4}$. It then follows that $\frac{1}{G} \sum_{g} \tilde{D}_{i, B, \delta}\left(z_{g} ; \bar{\delta}_{i}, \hat{\gamma}_{(1, i)}\right) \stackrel{p}{\longrightarrow} \mathcal{M}_{i, \delta}^{*}$ under condition D'-(i). Hence the second term on the right-hand side of $(12)$ is $\left[\mathcal{M}_{i, \delta}^{*}+o_{p}(1)\right] *\left[G^{-1 / 2} \sum_{g} \Psi_{\delta}^{i}\left(z_{g}\right)+o_{p}(1)\right]$, where $G^{-1 / 2} \sum_{g} \Psi_{\delta}^{i}\left(z_{g}\right)$ is $O_{p}(1)$. It then follows that

$$
G^{-\frac{1}{2}} \sum_{g} m_{B}^{i}\left(z_{g} ; \hat{\delta}_{i}, \hat{\gamma}_{(1, i)}\right)=G^{-\frac{1}{2}} \sum_{g}\left[m_{B}^{i}\left(z_{g} ; \delta_{i}, \hat{\gamma}_{(1, i)}\right)+\mathcal{M}_{i, \delta}^{*} \Psi_{\delta}^{i}\left(z_{g}\right)\right]+o_{p}(1) .
$$

This proves Lemma 3.1. Q.E.D.

Lemma 3.2 Under Assumptions S', K, B and D',

$$
\begin{aligned}
& \frac{1}{G} \sum_{g}\left[m_{B}^{i}\left(z_{g} ; \delta_{i}, \hat{\gamma}_{(1, i)}\right)-m_{B}^{i}\left(z_{g} ; \delta_{i}, \gamma_{(1, i)}^{*}\right)\right] \\
= & \int M_{B, \gamma}^{i}\left(z, \hat{\gamma}_{(1, i)}-\gamma_{(1, i)}^{*}\right)+M_{B, f}^{i}\left(z, \hat{\gamma}_{0}-\gamma_{0}^{*}\right) d F_{Z}^{*}+o_{p}\left(G^{-1 / 2}\right) .
\end{aligned}
$$

Proof of Lemma 3.2. Under conditions S'-(a), (b), we have

$$
\begin{aligned}
& \| \begin{array}{l}
m_{B}^{i}\left(z_{g} ; \delta_{i}, \hat{\gamma}_{(1, i)}\right)-m_{B}^{i}\left(z_{g} ; \delta_{i}, \gamma_{(1, i)}^{*}\right) \\
-M_{B, \gamma}^{i}\left(z_{g}, \hat{\gamma}_{(1, i)}-\gamma_{(1, i)}^{*}\right)-M_{B, f}^{i}\left(z_{g}, \hat{\gamma}_{0}-\gamma_{0}^{*}\right)
\end{array} \\
& \leq \quad b_{\gamma, f}\left(z_{g}\right) \sup _{x \in \Omega_{X}}\left\|\hat{\gamma}_{(1, i)}-\gamma_{(1, i)}^{*}\right\|^{2},
\end{aligned}
$$

for some non-negative function $b_{\gamma, f}$ (which may depend on true parameters in the DGP $\delta_{i}$ and $\left.\gamma_{(1)}^{*}\right)$ and $E\left[b_{\gamma, f}(Z)\right]<+\infty$. Under Assumptions S'-(a),(b), K and B, $\sup _{x \in \Omega_{X}}\left\|\hat{\gamma}_{(1, i)}-\gamma_{(1, i)}^{*}\right\|$ is $o_{p}\left(G^{-1 / 4}\right)$. Thus the right-hand side of the inequality in (13) is $o_{p}\left(G^{-1 / 2}\right)$. It follows from the triangular inequality and the Law of Large Numbers that:

$$
\frac{1}{G} \sum_{g}\left[\begin{array}{l}
m_{B}^{i}\left(z_{g} ; \delta_{i}, \hat{\gamma}_{(1, i)}\right)-m_{B}^{i}\left(z_{g} ; \delta_{i}, \gamma_{(1, i)}^{*}\right) \\
-M_{B, \gamma}^{i}\left(z_{g}, \hat{\gamma}_{(1, i)}-\gamma_{(1, i)}^{*}\right)-M_{B, f}^{i}\left(z_{g}, \hat{\gamma}_{0}-\gamma_{0}^{*}\right)
\end{array}\right]=o_{p}\left(G^{-\frac{1}{2}}\right)
$$

By construction,

$$
\begin{aligned}
& \frac{1}{G} \sum_{g} M_{B, \gamma}^{i}\left(z_{g}, \hat{\gamma}_{(1, i)}-\gamma_{(1, i)}^{*}\right)-\int M_{B, \gamma}^{i}\left(z ; \hat{\gamma}_{(1, i)}-\gamma_{(1, i)}^{*}\right) d F_{Z}^{*} \\
= & \frac{1}{G} \sum_{g} M_{B, \gamma}^{i}\left(z_{g}, \hat{\gamma}_{(1, i)}-\bar{\gamma}_{(1, i)}\right)-\int M_{B, \gamma}^{i}\left(z ; \hat{\gamma}_{(1, i)}-\bar{\gamma}_{(1, i)}\right) d F_{Z}^{*}+ \\
& \frac{1}{G} \sum_{g} M_{B, \gamma}^{i}\left(z_{g}, \bar{\gamma}_{(1, i)}-\gamma_{(1, i)}^{*}\right)-\int M_{B, \gamma}^{i}\left(z ; \bar{\gamma}_{(1, i)}-\gamma_{(1, i)}^{*}\right) d F_{Z}^{*}
\end{aligned}
$$


where $\bar{\gamma}_{(1, i)}(x) \equiv E\left[\hat{\gamma}_{(1, i)}(x)\right]$ is a function of $x$ (i.e. the expectation is taken with respect to the random sample used for estimating $\hat{\gamma}_{(1, i)}$ at $\left.x\right)$. For any $s, g \leq G$, define $a_{B, G}\left(z_{g}, z_{s}\right) \equiv$

$$
\left[\begin{array}{c}
K_{\sigma}\left(x_{s}-x_{g}\right), d_{s, j} K_{\sigma}\left(x_{s}-x_{g}\right), . . \\
K_{\sigma, i}\left(x_{s}-x_{g}\right), d_{s, j} K_{\sigma, i}\left(x_{s}-x_{g}\right)
\end{array}\right] \tilde{D}_{i, B}\left(z_{g}\right) .
$$

Let $a_{B, G}^{2}\left(z_{s}\right) \equiv \int a_{B, G}\left(z, z_{s}\right) d F_{Z}^{*}(z)$, and

$$
\begin{aligned}
a_{B, G}^{1}\left(z_{g}\right) & \equiv \int a_{B, G}\left(z_{g}, \hat{z}\right) d F_{Z}^{*}(\hat{z})=\int\left[\begin{array}{c}
K_{\sigma}\left(\hat{x}-x_{g}\right), \hat{d}_{j} K_{\sigma}\left(\hat{x}-x_{g}\right), . . \\
K_{\sigma, i}\left(\hat{x}-x_{g}\right), \hat{d}_{j} K_{\sigma, i}\left(\hat{x}-x_{g}\right)
\end{array}\right] \tilde{D}_{i, B}\left(z_{g}\right) d F_{Z}^{*}(\hat{z}) \\
& =\bar{\gamma}_{(1, i)}\left(x_{g}\right) \tilde{D}_{i, B}\left(z_{g}\right) .
\end{aligned}
$$

Then the first difference on the r.h.s. of (14) can be written as:

$$
G^{-2} \sum_{g} \sum_{s} a_{B, G}\left(z_{g}, z_{s}\right)-G^{-1} \sum_{g} a_{B, G}^{1}\left(z_{g}\right)-G^{-1} \sum_{g} a_{B, G}^{2}\left(z_{g}\right)+E\left[a_{B, G}^{1}(Z)\right] .
$$

Then by condition D'-(ii) and the boundedness of kernel function in Assumption $\mathrm{K}, E\left[\left\|a_{B, G}(Z, Z)\right\|\right]$ $\leq \sigma^{-J_{c}} C_{1}^{\prime}$ and $\left\{E\left[\left\|a_{B, G}\left(Z, Z^{\prime}\right)\right\|^{2}\right]\right\}^{1 / 2} \leq \sigma^{-J_{c}} C_{2}^{\prime}$, where $C_{1}^{\prime}, C_{2}^{\prime}$ are finite positive constants (whose form depend on the true parameters in $\gamma_{(1, i)}^{*}$ and the kernel function). Since $\sqrt{G} \sigma^{J_{c}} \longrightarrow+\infty$ under Assumption B, it follows from Lemma 8.4 in Newey and McFadden (1994) that the first difference is $o_{p}\left(G^{-1 / 2}\right)$. The boundedness of derivatives of $m_{B}^{i}$ w.r.t. $\gamma_{(1, i)}$ in D'-(ii) implies $E\left[\left\|M_{B, \gamma}^{i}\left(Z ; \bar{\gamma}_{(1, i)}-\gamma_{(1, i)}^{*}\right)\right\|^{2}\right] \leq C_{3}^{\prime} \sup _{x \in \Omega_{X}}\left\|\bar{\gamma}_{(1, i)}-\gamma_{(1, i)}^{*}\right\|$. Under Assumptions K and S'-(a), $\sup _{x \in \Omega_{X}}\left\|\bar{\gamma}_{(1, i)}-\gamma_{(1, i)}^{*}\right\|=o\left(\sigma^{m}\right)$, which converges to 0 as $\sigma \longrightarrow 0$ under Assumption B. It then follows from the Chebychev's Inequality that the second difference in (14) is $o_{p}\left(G^{-1 / 2}\right)$. We can also decompose the linear functional in the second term in a similar manner and have:

$$
\begin{aligned}
& \frac{1}{G} \sum_{g} M_{B, f}^{i}\left(z_{g}, \hat{\gamma}_{0}-\gamma_{0}^{*}\right)-\int M_{B, f}^{i}\left(z ; \hat{\gamma}_{0}-\gamma_{0}^{*}\right) d F_{Z}^{*} \\
= & \frac{1}{G} \sum_{g} M_{B, f}^{i}\left(z_{g}, \hat{\gamma}_{0}-\bar{\gamma}_{0}\right)-\int M_{B, f}^{i}\left(z ; \hat{\gamma}_{0}-\bar{\gamma}_{0}\right) d F_{Z}^{*}+ \\
& \frac{1}{G} \sum_{g} M_{B, f}^{i}\left(z_{g}, \bar{\gamma}_{0}-\gamma_{0}^{*}\right)-\int M_{B, f}^{i}\left(z ; \bar{\gamma}_{0}-\gamma_{0}^{*}\right) d F_{Z}^{*} .
\end{aligned}
$$

Arguments similar to those above suggest both differences in (15) are $o_{p}\left(G^{-1 / 2}\right)$ under conditions in Assumption S', K, B and D'. Q.E.D.

Lemma 3.3 Under Assumptions $S^{\prime}, K, B, D^{\prime}$ and $W^{\prime}$, for $i=1,2$ and $j=3-i$,

$$
\int M_{B, \gamma}^{i}\left(z, \hat{\gamma}_{(1, i)}-\gamma_{(1, i)}^{*}\right)+M_{B, f}^{i}\left(z, \hat{\gamma}_{0}-\gamma_{0}^{*}\right) d F_{Z}^{*}=\frac{1}{G} \sum_{g} \tilde{\nu}_{B}^{i}\left(z_{g}\right)+o_{p}\left(G^{-1 / 2}\right)
$$

where

$$
\tilde{\nu}_{B}^{i}\left(z_{g}\right) \equiv \psi_{B, 0}^{i}\left(x_{g}\right)+\psi_{B, f}^{i}\left(x_{g}\right)+\psi_{B, j}^{i}\left(x_{g}\right) d_{g, j}-E\left[\psi_{B, 0}^{i}(X)+\psi_{B, f}^{i}(X)+\psi_{B, j}^{i}(X) D_{j}\right] .
$$


Proof of Lemma 3.3. By smoothness of the true population function $\gamma_{(1, i)}^{*}$ in DGP in S'-(a), and that of the kernel function in S', K, D', and using the convexity of $\Omega_{X_{i} \mid x_{-i}}$ in W', we can apply integration-by-parts to write the left-hand side of the equation (16) as:

$$
\int\left[\begin{array}{l}
\hat{\gamma}_{0}(x)-\gamma_{0}^{*}(x) \\
\hat{\gamma}_{j}(x)-\gamma_{j}^{*}(x)
\end{array}\right]^{\prime}\left[\begin{array}{c}
\psi_{B, 0}^{i}(x) \\
\psi_{B, j}^{i}(x)
\end{array}\right]+\psi_{B, f}^{i}(x)\left[\hat{\gamma}_{0}(x)-\gamma_{0}^{*}(x)\right] d x,
$$

where $\psi_{B, 0}^{i}, \psi_{B, j}^{i}$ and $\psi_{B, f}^{j}$ are defined as in the text. (See the web appendix of this article for detailed derivations.) By arguments similar to those in the proof of Lemma 1.2, the expression in (17) is $\frac{1}{G} \sum_{g} \nu_{B}^{i}\left(z_{g}\right)+o_{p}\left(G^{-\frac{1}{2}}\right)$ due to conditions in Assumptions S', K, B and D' and that $\psi_{B, f}^{i}(x)$ is almost everywhere continuous in $x$. Q.E.D.

By Lemma 3.1, Lemma 3.2 and Lemma 3.3, we have

$$
\hat{\mu}_{i, \Psi} \equiv \frac{1}{G} \sum_{g} m_{B}^{i}\left(z_{g} ; \hat{\delta}_{i}, \hat{\gamma}_{(1, i)}\right)=\frac{1}{G} \sum_{g} \Psi_{B}^{i}\left(z_{g}\right)+o_{p}\left(G^{-\frac{1}{2}}\right)
$$

where

$$
\Psi_{B}^{i}(z) \equiv m_{B}^{i}\left(z ; \delta_{i}, \gamma_{(1, i)}^{*}\right)+\tilde{\nu}_{B}^{i}(z)+\mathcal{M}_{i, \delta}^{*} \Psi_{\delta}^{i}(z)
$$

By construction

$$
E\left[\Psi_{B}^{i}(Z)\right]=E\left[m_{B}^{i}\left(Z ; \delta_{i}, \gamma_{(1, i)}^{*}\right)\right] \equiv \mu_{i, \Psi}
$$

Let $\hat{\lambda} \equiv \frac{1}{G} \sum_{g} \tilde{x}_{g}^{\prime} \tilde{x}_{g}$ and $\lambda \equiv E\left(\tilde{X}^{\prime} \tilde{X}\right)$. By definition, $\beta_{i}=\lambda^{-1} \mu_{i, \Psi}$ and

$$
\hat{\beta}_{i}=\hat{\lambda}^{-1}\left(\frac{1}{G} \sum_{g} \Psi_{B}^{i}\left(z_{g}\right)+o_{p}\left(G^{-1 / 2}\right)\right),
$$

where $\hat{\lambda}^{-1}=\lambda^{-1}+o_{p}(1)$. Note

$$
\begin{aligned}
& \hat{\beta}_{i}=\hat{\lambda}^{-1}\left(\hat{\mu}_{i, \Psi}-\hat{\lambda} \beta_{i}+\hat{\lambda} \beta_{i}\right)=\hat{\lambda}^{-1}\left(\hat{\mu}_{i, \Psi}-\hat{\lambda} \beta_{i}\right)+\beta_{i} \\
\Rightarrow \quad & \sqrt{G}\left(\hat{\beta}_{i}-\beta_{i}\right)=\hat{\lambda}^{-1} \sqrt{G}\left(\hat{\mu}_{i, \Psi}-\hat{\lambda} \beta_{i}\right) .
\end{aligned}
$$

where

$$
\sqrt{G}\left(\hat{\mu}_{i, \Psi}-\hat{\lambda} \beta_{i}\right)=\frac{1}{\sqrt{G}} \sum_{g}\left(\Psi_{B}^{i}\left(z_{g}\right)-\tilde{x}_{g}^{\prime} \tilde{x}_{g} \beta_{i}\right)+o_{p}(1) .
$$

By construction, $E\left[\Psi_{B}^{i}(Z)-\tilde{X}^{\prime} \tilde{X} \beta_{i}\right]=0$. Under conditions in S', D' and R', $E\left[\left\|\Psi_{B}^{i}(Z)-\tilde{X}^{\prime} \tilde{X} \beta_{i}\right\|^{2}\right]<$ $+\infty$. Then the Central Limit Theorem and the Slutsky Theorem can be applied to show that $\sqrt{G}\left(\hat{\beta}_{i}-\beta_{i}\right) \stackrel{d}{\rightarrow} \mathcal{N}\left(0, \Sigma_{B}^{i}\right)$ with

$$
\Sigma_{B}^{i} \equiv \lambda^{-1} \operatorname{Var}\left(\Psi_{B}^{i}(Z)-\tilde{X}^{\prime} \tilde{X} \beta_{i}\right)\left(\lambda^{-1}\right)^{\prime}
$$

This completes the proof of the limiting distribution of $\sqrt{G}\left(\hat{\beta}_{i}-\beta_{i}\right)$. 


\section{Limiting distribution of $\sqrt{G}\left(\hat{T}(\bar{\omega})-\mu_{\bar{\omega}}\right)$}

Define

$$
v(x) \equiv \frac{1\{x \in \bar{\omega}\}}{\gamma_{0}^{*}(x)}\left[2 p_{1}^{*}(x) p_{2}^{*}(x),-p_{2}^{*}(x),-p_{1}^{*}(x)\right]
$$

For notation simplicity, we suppress dependence of $\hat{T}$ on $\bar{\omega}$ when there is no confusion. We now collect the appropriate conditions for establishing the asymptotic properties of $\hat{T}$.

Assumption T1 (i) The true density $\gamma_{0}^{*}(x)$ is bounded away from zero and $\gamma_{k}^{*}(x)$ are bounded above over $\bar{\omega}$ for $k=1,2$. (ii) For any $\tilde{x}$ and $i=1,2, \gamma_{0}^{*}\left(x_{e} \mid \tilde{x}\right)$ and $p_{i}^{*}(x) \gamma_{0}^{*}\left(x_{e} \mid \tilde{x}\right)$ are continuously differentiable of order $m \geq 2$, and the derivatives of order $m \geq 2$ are uniformly bounded over $\bar{\omega}$. (iii) there exists $\eta>0$ such that $\left[V A R_{u_{i}}(x)\right]^{1+\eta} \gamma_{0}^{*}\left(x_{e} \mid \tilde{x}\right)$ (where $V A R_{u_{i}}(x)$ denotes variance of $u_{i}=d_{i}-p_{i}^{*}(x)$ conditional on $\left.x\right)$ is uniformly bounded; and (iv) both $p_{i}^{*}(x)^{2} \gamma_{0}^{*}\left(x_{e} \mid \tilde{x}\right)$ and $V A R_{u_{i}}(x) \gamma_{0}^{*}\left(x_{e} \mid \tilde{x}\right)$ are continuous and bounded over the support of $X_{e}$ given $\tilde{x}$.

Assumption T2 $K($.$) is a product kernel function with support in \mathbb{R}^{2}$ such that (i) $\int\left|K\left(t_{1}, t_{2}\right)\right| d t_{1} d t_{2}<$ $\infty$ and $\int K\left(t_{1}, t_{2}\right) d t_{1} d t_{2}=1$; and (ii) $K($.$) has zero moments up to the order of m$, and $\int\|u\|^{m}$ $\left|K\left(t_{1}, t_{2}\right)\right| d t_{1} d t_{2}<\infty$.

Assumption T3 $\sqrt{G} \sigma^{m} \rightarrow 0$ and $\left(\frac{\sqrt{G}}{\ln G}\right) \sigma^{2} \rightarrow+\infty$ as $G \rightarrow+\infty$.

Assumption T4 (i) Given any $\tilde{x}, v$ is continuous almost everywhere w.r.t. Lebesgue measure on the support of $\left(X_{1}, X_{2}\right)$. (ii) There exists $\varepsilon>0$ such that $E\left[\sup _{\|\eta\| \leq \varepsilon}\|v(x+\eta)\|^{4}\right]<\infty$.

Conditions in T1 ensure $v$ is bounded over any bounded subset of $\Omega_{X}$. Conditions in T2, T3 together guarantee that the kernel estimators $\hat{\gamma}$ are well-behaved in the sense $\sup _{x \in \bar{\omega}}\left\|\hat{\gamma}-\gamma^{*}\right\|=$ $o_{p}\left(G^{-1 / 4}\right)$ and $\sup _{x \in \bar{\omega}}\left\|E(\hat{\gamma})-\gamma^{*}\right\| \longrightarrow 0$ as $G \rightarrow \infty$.

Lemma 4.1 Suppose assumptions T1,2,3 hold. Then

$$
\hat{T}(\bar{\omega})-\frac{1}{G} \sum_{g} 1\left\{x_{g} \in \bar{\omega}\right\} m\left(z_{g} ; \gamma^{*}\right)=\int M\left(z, \hat{\gamma}-\gamma^{*}\right) d F_{Z}^{*}+o_{p}\left(G^{-1 / 2}\right)
$$

where $M(z, \gamma) \equiv v(x) \gamma(x)$ with $v($.$) defined in (18) above.$

Proof of Lemma 4.1. We will drop the dependence of $\hat{T}$ and $T$ on $\omega$ for notational simplicity. It follows from linearization arguments and $T 1$-(i) that there exists a constant $C<\infty$ so that for any $\gamma \equiv\left[\gamma_{0}, \gamma_{1}, \gamma_{2}\right]^{\prime}$ with $\sup _{x \in \bar{\omega}}\left\|\gamma-\gamma^{*}\right\|$ small enough,

$$
1\{x \in \bar{\omega}\}\left|m(z ; \gamma)-m\left(z ; \gamma^{*}\right)-M\left(z, \gamma-\gamma^{*}\right)\right| \leq C \sup _{x \in \bar{\omega}}\left\|\gamma-\gamma^{*}\right\|^{2}
$$

for all $z$. Given $T 1,2,3$, we have $\sup _{x \in \bar{\omega}}\left\|\hat{\gamma}-\gamma^{*}\right\|=O_{p}\left(\sigma^{-m /(2 m+4)}\right)$ by Theorem 3.2.1. in Bierens (1987). For $m \geq 2$ (the parameter $m$ captures smoothness properties of population moments in 
T1,T2),

$$
\frac{1}{G} \sum_{g} 1\left\{x_{g} \in \bar{\omega}\right\}\left[m\left(z_{g} ; \hat{\gamma}\right)-m\left(z_{g} ; \gamma^{*}\right)\right]-M\left(z_{g}, \hat{\gamma}-\gamma^{*}\right)=o_{p}\left(G^{-1 / 2}\right)
$$

Next, define $\bar{\gamma} \equiv E(\hat{\gamma})$ and by the linearity of $M(z, \gamma)$ in $\gamma$, we can write:

$$
\begin{aligned}
& G^{-1} \sum_{g} M\left(z_{g}, \hat{\gamma}-\gamma^{*}\right)-\int M\left(z, \hat{\gamma}-\gamma^{*}\right) d F^{*}(z) \\
= & G^{-1} \sum_{g} M\left(z_{g}, \hat{\gamma}-\bar{\gamma}\right)-\int M(z, \hat{\gamma}-\bar{\gamma}) d F^{*}(z) \\
& +G^{-1} \sum_{g} M\left(z_{g}, \bar{\gamma}-\gamma^{*}\right)-\int M\left(z, \bar{\gamma}-\gamma^{*}\right) d F^{*}(z)
\end{aligned}
$$

Under $T 1$, there exists a function $c$ such that $\|M(z, \gamma)\| \leq c(z) \sup _{x \in \bar{\omega}}\|\gamma\|$ with $E\left[c(z)^{2}\right]<\infty$. (This is because $w$ is an indicator function, $\gamma_{i}^{*} / \gamma_{0}^{*}=p_{i}^{*}$ are bounded above by 1 for $i=1,2$ and $1 / \gamma_{0}^{*}$ is bounded above over the relevant support in T1.) By the Theorem of Projection for $V$-statistics (Lemma 8.4 of Newey and McFadden (1994)) and that $G \sigma^{2} \rightarrow \infty$ in T3, the first difference in (22) is $o_{p}\left(G^{-1 / 2}\right)$. Next, note by the Chebyshev's inequality,

$$
\operatorname{Pr}\left\{\sqrt{G}\left|G^{-1} \sum_{g} M\left(z_{g}, \bar{\gamma}-\gamma^{*}\right)-\int M\left(z, \bar{\gamma}-\gamma^{*}\right) d F^{*}(z)\right| \geq \varepsilon\right\} \leq \frac{E\left[\left\|M\left(z_{g}, \bar{\gamma}-\gamma^{*}\right)\right\|^{2}\right]}{\varepsilon^{2}}
$$

But by construction, $E\left[\left\|M\left(z ; \bar{\gamma}-\gamma^{*}\right)\right\|^{2}\right] \leq c(z) \sup _{x \in \bar{\omega}}\left\|\bar{\gamma}-\gamma^{*}\right\|^{2}$. By smoothness conditions in T1-(ii) and properties of $K$ in T2, $\sup _{x \in \bar{\omega}}\left\|\bar{\gamma}-\gamma^{*}\right\|=O\left(\sigma^{m}\right)$. Hence the second difference in (22) is also $o_{p}\left(G^{-1 / 2}\right)$. Therefore,

$$
G^{-1} \sum_{g} M\left(z_{g}, \hat{\gamma}-\gamma^{*}\right)-\int M\left(z, \hat{\gamma}-\gamma^{*}\right) d F^{*}(z)=o_{p}\left(G^{-1 / 2}\right)
$$

This and (21) together proves (19) in the lemma. Q.E.D.

Lemma 4.2 Suppose T1,2,3,4 hold. Then $\int M\left(z, \hat{\gamma}-\gamma^{*}\right) d F_{Z}^{*}=\frac{1}{G} \sum_{g} \delta\left(z_{g}\right)+o_{p}\left(G^{-1 / 2}\right)$, where $\delta(z) \equiv v(x) y-E[v(X) Y]$

Proof of Lemma 4.2. By construction,

$$
\begin{aligned}
& \int M\left(z, \hat{\gamma}-\gamma^{*}\right) d F_{Z}^{*}=\int v(x)\left[\hat{\gamma}(x)-\gamma^{*}(x)\right] d x=\int v(x) \hat{\gamma}(x) d x-\int v(x) \gamma^{*}(x) d x \\
= & G^{-1} \sum_{g} \int v(x) y_{g} K_{\sigma}\left(x-x_{g}\right) d x-\int v(x)\left[1, p_{1}^{*}(x), p_{2}^{*}(x)\right]^{\prime} \gamma_{0}^{*}(x) d x \\
= & \left.G^{-1} \sum_{g} \int v(x) y_{g} K_{\sigma}\left(x-x_{g}\right) d x-E[v(X) Y]=G^{-1} \sum_{g} \int \delta\left(x, d_{g, 1}, d_{g, 2}\right) K_{\sigma}\left(x-x_{g}\right) d x 24\right)
\end{aligned}
$$

where the last equality uses $\int K_{\sigma}\left(x-x_{g}\right) d x=1$ for all $x_{g}$ (which follows from change-of-variables). Define a probability measure $\hat{F}$ on the support of $X$ such that

$$
\int a\left(x, d_{1}, d_{2}\right) d \hat{F} \equiv G^{-1} \sum_{g} \int a\left(x, d_{g, 1}, d_{g, 2}\right) K_{\sigma}\left(x-x_{g}\right) d x
$$


for any function $a$ over the support of $X$ and $\{0,1\}^{2}$. Thus (24) can be written as $\int M\left(z, \hat{\gamma}-\gamma^{*}\right) d F^{*}$ $=\int \delta(z) d \hat{F}$.

Let $K_{\sigma}^{*}\left(x-x_{g}\right) \equiv \sigma^{-2} \Pi_{i=1,2} K_{i}\left(\frac{x_{i}-x_{g, i}}{\sigma}\right) 1\left(\tilde{x}=\tilde{x}_{g}\right)$, where $K_{i}($.$) denote univariate kernels$ corresponding to coordinates $x_{i}$ for $i=1,2$. Thus:

$$
\begin{aligned}
& \int \delta(z) d \hat{F}-G^{-1} \sum_{g} \delta\left(z_{g}\right) \\
=G^{-1} \quad & \sum_{g}\left(\int v(x) y_{g} K_{\sigma}\left(x-x_{g}\right)-v(x) y_{g} K_{\sigma}^{*}\left(x-x_{g}\right) d x\right) \\
+G^{-1} & \sum_{g}\left(\int v(x) y_{g} K_{\sigma}^{*}\left(x-x_{g}\right) d x-v\left(x_{g}\right) y_{g}\right)
\end{aligned}
$$

By the definition of $v(x)$ and properties of $K$ and $\sigma$ in $T 1-4$, the first term on the right-hand side of $(25)$ is $o_{p}\left(G^{-1 / 2}\right)$. The second term is also $o_{p}\left(G^{-1 / 2}\right)$ through application of the Dominated Convergence Theorem, using the properties of $v$ in $T_{4}$ and its boundedness over any bounded subsets of $\Omega_{X}$. (See Theorem 8.11 in Newey and McFadden (1994) for details.) Q.E.D.

Proof of Theorem 6 in Lewbel and Tang (2012). By Lemma 4.1 and 4.2,

$$
\hat{T}(\bar{\omega})=\frac{1}{G} \sum_{g}\left[1\left\{x_{g} \in \bar{\omega}\right\} m\left(z_{g} ; \gamma^{*}\right)+\delta\left(z_{g}\right)\right]+o_{p}\left(G^{-\frac{1}{2}}\right)
$$

By the Central Limit Theorem, $\sqrt{G}\left(\hat{T}(\bar{\omega})-\mu_{\bar{\omega}}\right) \stackrel{d}{\longrightarrow} N\left(0, \Sigma_{\bar{\omega}}\right)$, where

$$
\begin{aligned}
\Sigma_{\bar{\omega}} & \equiv \operatorname{Var}\left[1\{X \in \bar{\omega}\} m\left(Z ; \gamma^{*}\right)+\delta(Z)\right] \\
& =\operatorname{Var}\left[1\{X \in \bar{\omega}\}\left(D_{1} D_{2}-p_{1}^{*}(X) p_{2}^{*}(X)+\frac{2 p_{1}^{*}(X) p_{2}^{*}(X)-D_{1} p_{2}^{*}(X)-D_{2} p_{1}^{*}(X)}{\gamma_{0}^{*}(X)}\right)\right]
\end{aligned}
$$

This proves the theorem. Q.E.D.

\section{References}

[1] Aradillas-Lopez, A., "Semiparametric Estimation of a Simultaneous Game with Incomplete Information", Journal of Econometrics, Volume 157 (2), Aug 2010, pp: 409-431

[2] Bierens, H., "Kernel Estimators of Regression Functions", in: Truman F.Bewley (ed.), Advances in Econometrics: Fifth World Congress, Vol.I, New York: Cambridge University Press, 1987, 99-144.

[3] Lewbel, A. and X. Tang, "Identification and Estimation of Games with Incomplete Information Using Excluded Regressors," unpublished manuscript, 2012

[4] Newey, W. and D. McFadden, "Large Sample Estimation and Hypothesis Testing,", Handbook of Econometrics, Vol 4, ed. R.F.Engle and D.L.McFadden, Elsevier Science, 1994 